Medidas transversas, correntes e sistemas dinâmicos

Jorge Luis Crisostomo Parejas 

SERVIÇO DE PÓS-GRADUAÇÃO DO ICMC-USP

Data de Depósito:

Assinatura:

\title{
Medidas transversas, correntes e sistemas dinâmicos
}

\author{
Jorge Luis Crisostomo Parejas
}

Orientador: Prof. Dr. Ali Tahzibi

Dissertação apresentada ao Instituto de Ciências Matemáticas e de Computação - ICMC-USP, como parte dos requisitos para obtenção do título de Mestre em Ciências - Matemática . VERSÃO REVISADA 
Ficha catalográfica elaborada pela Biblioteca Prof. Achille Bassi e Seção Técnica de Informática, ICMC/USP, com os dados fornecidos pelo(a) autor(a)

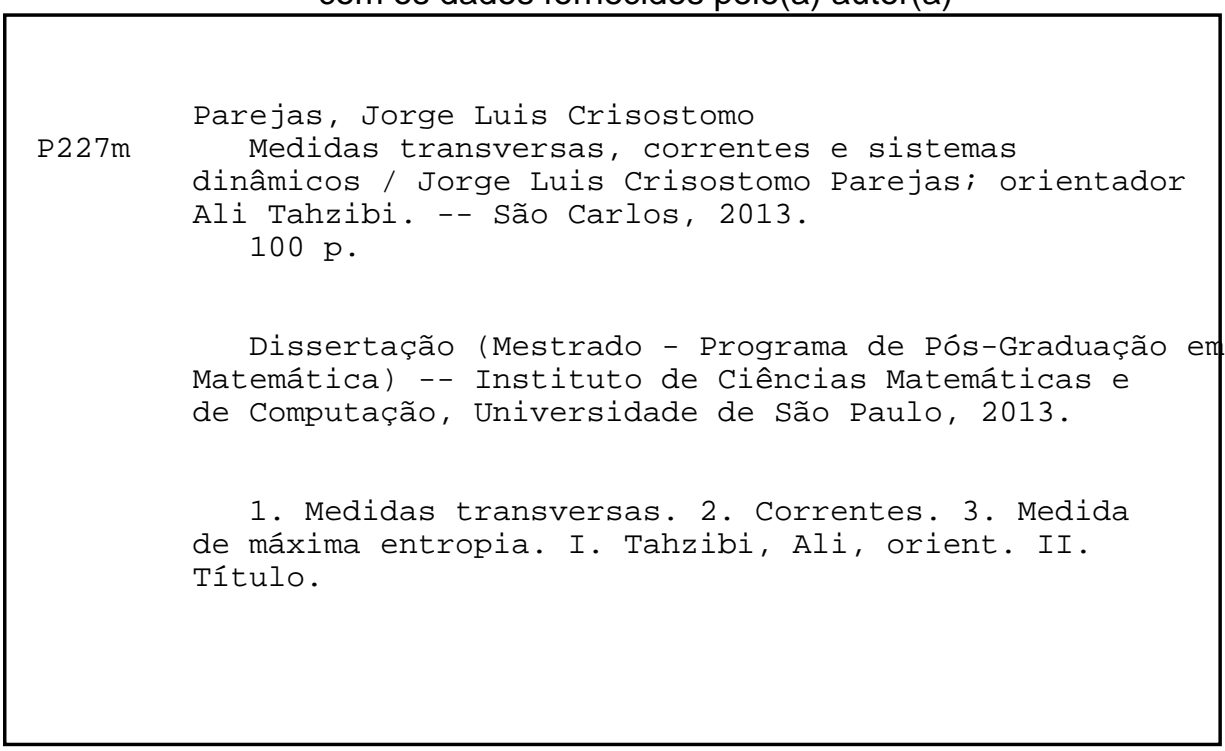


À minha família. 
Não conseguimos encontrar respostas para todo os nossos problemas. As que encontramos apenas nos levaram a formular novas questões. De uma certa maneira sentimonos tão confusos como antes, mas acreditamos que agora estamos confusos num nivel mais alto e sobre coisas mais importantes.

Encontrado na porta do Instituto de Matemática na Universidade de Tromso-Noruega 


\section{Agradecimentos}

Ao meu orientador Ali Tahzibi, pelos momentos de discussão frutífera e pelo enriquecimento da minha experiencia matemática.

Aos meus pais, Herminio e Julia, ao meus irmãos e irmas, por terem sempre me apoiado nas minhas decisões, por terem contribuído de forma fundamental na minha formação pessoal.

À minha noiva Luz, por ter sido compreensiva com minha ausência durante o período do mestrado e por ter me dado ânimos para prosseguir diante das intempéries.

Ao meus amigos peruanos e brasileiros, meus orientadores da graduação. Graças a eles, descobri o meu interesse pela matemática e pude receber um treinamento na minha graduação que me permitiu estar aqui hoje.

Aos professores do instituto que, com toda certeza, contribuíram para meu crescimento profissional, em especial para os professores: Ederson, Tengan e Carlos Maquera.

À CNPQ pelo suporte financeiro. 


\section{Resumo}

Neste trabalho, fazemos um estudo das correntes e das medidas transversas invariantes por holonomia, e mostraremos o resultado de D. Sullivan [23] sobre a correspondência biunívoca entre estes dois objetos. Em particular mostraremos um resultado conhecido de J. Plante [17] sobre a existência de medidas transversas invariantes sob a hipótese de crescimento sub-exponencial.

Apresentamos também, o resultado devido a Ruelle-Sullivan [19] de que a medida de máxima entropia de um difeomorfismo topologicamente mixing pode-se expressar como o produto de duas medidas transversas invariantes para as folheações estáveis e instáveis. Por último, mostramos que os difeomorfismos de Anosov topologicamente mixing, que preservam a orientação das folhas estáveis e folhas instáveis induzem elementos da cohomologia de DeRham.

Palavras-chaves: Medidas transversas invariantes, cohomologia de DeRham, correntes, difeomorfismos e medida de máxima entropia. 


\section{Abstract}

In this work, we make a study of currents and holonomy invariant transverse measure, and we will show the result of D. Sullivan [23] about the biunivocal correspondence between these two objects. In particular we show a known result of J. Plante [17] about the existence of invariant transverse measures under the hypothesis of sub-exponential growth.

Also we will present, the result due to Ruelle-Sullivan [19] that the maximum entropy measure of a diffeomorphism topologically mixing can be expressed as the product of two invariant transverse measures for stable and unstable foliations. Finally, we show that the Anosov diffeomorphisms topologically mixing, which preserve the orientation of the leaves stable and unstable induce elements DeRham cohomology.

Keywords: Invariant transverse measure, DeRham cohomology, currents, diffeomorphism and maximum entropy measure. 


\section{Sumário}

Agradecimentos $\quad$ i

Resumo iii

Abstract $\quad$ v

Introdução 1

1 Preliminares 5

1.1 Folheações . . . . . . . . . . . . . . . . . . . . 5 5

1.2 Espaços fibrados . . . . . . . . . . . . . . . . . . 7

1.3 Dinâmica hiperbólica . . . . . . . . . . . . . . . . . . . 9 9

1.4 Partições de Markov . . . . . . . . . . . . . . . . . . . . . 11

1.5 Entropia . . . . . . . . . . . . . . . . . 14

1.5.1 Entropia com respeito a uma medida . . . . . . . . . . . . 14

1.5.2 Entropia topológica . . . . . . . . . . . . . . . 15

1.6 Medida de Markov . . . . . . . . . . . . . . . . . . . . . . . . 17

2 Medidas transversas invariantes por holonomia 21

2.1 Medida transversa invariante . . . . . . . . . . . . . . . . . . . . . . 21

2.2 Crescimento de funções . . . . . . . . . . . . . . . . . . . . . . . . . . 29

2.3 Condições suficientes para a existência de uma medida invariante . . . . . 31

2.4 Crescimento em folheações . . . . . . . . . . . . . . . . . . . 39

2.4.1 Crescimento de folhas desde um ponto de vista geométrico . . . . . 41

$\begin{array}{llr}3 & \text { Correntes } & 47\end{array}$

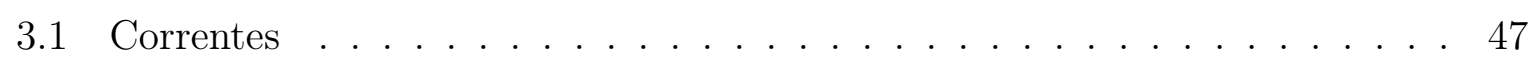

3.2 Corrente Folheada e ciclos folheados . . . . . . . . . . . . . . . . . 54 
3.3 As medidas transversas invariantes são ciclos folheados . . . . . . . . . . . 58

4 Exemplos Dinâmicos $\quad 65$

4.1 Thom-Arnold map . . . . . . . . . . . . . . . . . . 65

4.2 Ferradura de Smale . . . . . . . . . . . . . . . . . . . . . . . . 69

4.2 .1 Dinâmica simbólica . . . . . . . . . . . . . . . . . . . . . 69

4.2 .2 Ferradura de Smale . . . . . . . . . . . . . . . . . . . 71

5 Correntes e Difeomorfismos $\quad 77$

5.1 Medida de máxima entropia . . . . . . . . . . . . . . 77

5.2 Medidas transversas e Sistemas dinâmicos . . . . . . . . . . . . . . 85

$\begin{array}{ll}\text { A Seminormas que geram uma topologia } & 95\end{array}$

$\begin{array}{ll}\text { Referências Bibliográficas } & 99\end{array}$ 


\section{Introdução}

As medidas invariantes por um fluxo é um objeto de grande importância e utilidade para estudar a dinâmica dos fluxos. A generalização destas medidas em folheações é chamada medidas transversas invariantes por holonomia e é obtida considerando medidas no espaço transverso à folheação, onde se identificam os pontos que pertencem a uma mesma folha e pedindo que sejam invariantes sob o "fluxo das folhas". As medidas transversas têm sido objeto de estudo desde os anos 50 e demostraram ser muito úteis para a compreensão da dinâmica das folheações. No capítulo 2, fazemos um estudo destas medidas seguindo o artigo de J. Plante [17]. Neste capítulo, definiremos rigorosamente as medidas transversas invariantes por holonomia, demonstraremos um bonito teorema, que pode ser pensado com um teorema abstrato de Poincaré-Bendixson para folheações, e mostraremos também que a existência de uma medida transversa invariante induz uma aplicação linear o qual é chamada "corrente". Um dos objetivos deste trabalho, é mostrar um resultado conhecido de J. Plante [17] sobre a existência de medidas transversas invariantes sob a hipótese de crescimento sub-exponencial, especificamente mostramos o seguinte teorema.

Teorema 0.1 (J. Plante, [17]): Seja $\mathcal{F}$ uma folheação de codimensão $k$ de uma variedade compacta $M$. Se L é uma folha de $\mathcal{F}$ que tem crescimento não exponencial, então existe uma medida não trivial invariante pelo pseudogrupo de holonomia que é finito em conjuntos compactos e que tem suporte contido no fecho de L.

Para o qual introduzimos os conceitos de crescimento de funções e crescimento em folheações, este último é definido de duas maneiras, uma que é puramente algébrica e a outra geométrica, e demonstraremos que são equivalentes.

A teoria de correntes de DeRham [8] elaborada nos anos 50 é um análogo da teoria de distribuições de Schwartz [20] criada nos finais de 1940. As distribuições são funcionais contínuas definidas no espaço das funções com suporte compacto. Da mesma maneira, 
as $p$-correntes se definem como funcionais contínuas definidas no espaço das $p$-formas diferenciais com suporte compacto $\Omega_{c}^{p}$; e.g., as distribuições são 0-correntes. Munido com uma topologia adequada, a derivada exterior torna-se um operador linear continuo, pelo que podemos definir seu operador adjunto e a partir deste definir a homologia de DeRham. No capítulo 3, fazemos uma apresentação das correntes seguindo os livros [7] e [8]. Neste capítulo o resultado principal é provar o teorema de D. Sullivan [23] de que as medidas transversas invariantes estão em correspondência biunívoca com as correntes(ciclos folheados), especificamente mostramos o seguinte teorema.

Teorema 0.2 (D. Sullivan, [23]): Seja $\mathcal{F}$ uma folheação de dimensão p, transversalmente orientável e de classe $C^{1}$ de uma n-variedade compacta $M$ de classe $C^{\infty}$. Então as medidas transversas invariantes por holonomia e os ciclos folheados estão em correspondência canônica um a um.

O conhecido Principio Variacional afirma que se $f: X \rightarrow X$ é uma aplicação continua do espaço métrico compacto $X$, então o supremo das entropias métricas sobre o conjunto, das medidas de probabilidade invariantes coincidem com a entropia topológica.

$$
h_{t o p}(f)=\sup _{\mu \in M_{f}(X)} h_{\mu}(f)
$$

onde $M_{f}(X)$ é o conjunto de medidas de probabilidade invariantes. É um problema antigo e muito importante em saber se o supremo é um máximo, e neste caso se o máximo é alcançado em uma única medida de probabilidade ou não. R. Bowen, dá condições suficientes para responder positivamente a esta questão. De fato o R. Bowen [3] mostra que se $X$ é um espaço métrico compacto e $f: X \rightarrow X$ um homeomorfismo expansivo com a propriedade de especificação, existe uma única medida de probabilidade $f$-invariante que maximiza a entropia métrica.

Neste trabalho, apresentaremos a prova deste resultado, também devido R. Bowen [4], [5], para difeomorfismos topologicamente mixing, para o qual relacionamos o difeomorfismo hiperbólico com o shifts de tipo finito usando partições de Markov.

Nosso objetivo principal deste trabalho é apresentar o trabalho de Ruelle-Sullivan [19], onde se prova que a medida que maximiza a entropia métrica, pode-se expressar como o produto de duas medidas transversas invariantes para as folheações estáveis e instáveis. Para ser mais precisos mostramos o seguinte teorema.

Teorema 0.3 (D. Ruelle, D. Sullivan, [19]): Seja $f: M \rightarrow M$ um difeomorfismo $e$ $\Lambda \subset M$ um conjunto hiperbólico. Se $\left.f\right|_{\Lambda}$ que é topologicamente mixing. Então para cada 
$x \in \Lambda$ existe uma medida $\mu_{x}^{s}$ em $W_{\epsilon}^{s}(x)$ e uma medida $\mu_{x}^{u}$ em $W_{\epsilon}^{u}(x)$ tal que:

i) $\operatorname{supp}_{x}^{s} \subset W_{\epsilon}^{s}(x) \cap \Lambda$, supp $\mu_{x}^{u} \subset W_{\epsilon}^{u}(x) \cap \Lambda$.

ii) Se $d\left(x, x^{\prime}\right)<\delta$, então

$$
\left(P_{x, x^{\prime}}^{s}\right)_{*}\left(\left.\mu_{x^{\prime}}^{s}\right|_{W_{\delta}^{s}\left(x^{\prime}\right) \cap \Lambda}\right)=\left.\mu_{x}^{s}\right|_{P_{x, x^{\prime}}^{s}\left(W_{\delta}^{s}\left(x^{\prime}\right) \cap \Lambda\right)}
$$

analogamente

$$
\left(P_{x, x^{\prime}}^{u}\right)_{*}\left(\left.\mu_{x^{\prime}}^{u}\right|_{W_{\delta}^{u}\left(x^{\prime}\right) \cap \Lambda}\right)=\left.\mu_{x}^{u}\right|_{P_{x, x^{\prime}}^{u}}\left(W_{\delta}^{u}\left(x^{\prime}\right) \cap \Lambda\right)
$$

iii)

$$
\left.\left(f_{*}^{-1} \mu_{x}^{s}-\lambda^{-1} \mu_{f^{-1}(x)}^{s}\right)\right|_{W_{\epsilon}^{s}\left(f^{-1}(x)\right)}=0
$$

$e$

$$
\left.\left(f_{*} \mu_{x}^{u}-\lambda^{-1} \mu_{f(x)}^{u}\right)\right|_{W_{\epsilon}^{u}(f(x))}=0
$$

iv) Seja $[.,]:.\left(W_{\epsilon}^{u}(x) \cap \Lambda\right) \times\left(W_{\epsilon}^{s}(x) \cap \Lambda\right) \rightarrow \Lambda$ definida por $\{[y, z]\}=W_{\epsilon}^{s}(y) \cap W_{\epsilon}^{u}(z)$ então

$$
\left.[., .]_{*}\left(\mu_{x}^{u} \times \mu_{x}^{s}\right)\right|_{\left(W_{\epsilon}^{u}(x) \times W_{\epsilon}^{s}(x)\right) \cap \Lambda}=\left.\rho\right|_{\left(W_{\epsilon}^{u}(x) \times W_{\epsilon}^{s}(x)\right) \cap \Lambda}
$$

onde $\rho$ é a medida de Bowen em $\Lambda$.

Um último objetivo deste trabalho é juntar os três conceitos estudados neste trabalho: medidas transversas, correntes e sistemas dinâmicos. Especificamente provamos o seguinte teorema devido a Ruelle-Sullivan [19].

Teorema 0.4 (D. Ruelle, D. Sullivan, [19]): Se $f: M \rightarrow M$ é um difeomorfismo de Anosov topologicamente mixing, preservando a orientação das folhas estáveis e folhas instáveis, então existem $C^{s} k$-corrente e $C^{u}(n-k)$-corrente tal que

i) $C^{s}$ e $C^{u}$ são fechados e definem classes de cohomologia de DeRham $\left[C^{s}\right]$ e $\left[C^{s}\right]$ em $H^{n-k}(M)$ e $H^{k}(M)$ respectivamente.

ii) $f_{*} C^{s}=\frac{1}{\lambda} C^{s}$ e $f_{*} C^{u}=\lambda C^{u}$ onde $\log \lambda$ é a entropia topológica de $f$.

No capítulo 4, apresentamos dois exemplos de sistemas dinâmicos básicos: ThomArnold map e a ferradura de Smale e estudamos suas riqueza dinâmica. No capitulo 5, estudamos os dois objetivos principais deste trabalho, mencionados acima. O trabalho contém um apêndice, onde fazemos uma apresentação de topologia gerado por uma família de semi-normas. 


\section{Capítulo}

\section{Preliminares}

\subsection{Folheações}

Esta seção em sua maioria é extraída de [6]. Uma folheação de dimensão $p$ de uma variedade $M^{n}$ é, a grosso modo, uma decomposição de $M$ em subvariedades conexas de dimensão $p$ chamadas folhas, as quais se aglomeram localmente como os subconjuntos de $\mathbb{R}^{n}=\mathbb{R}^{p} \times \mathbb{R}^{n-p}$ com segunda coordenada constante.

Definição 1.1: Seja $M$ uma variedade de dimensão $n$ e classe $C^{\infty}$. Uma folheação de classe $C^{r}$ e dimensão $p$ em $M$, é um atlas máximo $\mathcal{F}$ de classe $C^{r}$ em $M$ com as seguintes propriedades:

a) Se $(U, \varphi) \in \mathcal{F}$ então $\varphi(U)=U_{1} \times U_{2} \subset \mathbb{R}^{p} \times \mathbb{R}^{n-p}$, onde $U_{1}$ e $U_{2}$ são discos abertos de $\mathbb{R}^{p}$ e de $\mathbb{R}^{n-p}$ respectivamente.

b) Se $(U, \varphi)$ e $(V, \psi) \in \mathcal{F}$ são tais que $U \cap V \neq \emptyset$ então a mudança de coordenadas $\psi \circ \varphi^{-1}: \varphi(U \cap V) \rightarrow \psi(U \cap V)$ é da forma $\psi \circ \varphi^{-1}(x, y)=\left(h_{1}(x, y), h_{2}(y)\right)$. As cartas $(U, \varphi) \in \mathcal{F}$ são chamadas cartas trivializadoras de $\mathcal{F}$.

Seja $\mathcal{F}$ uma folheação de classe $C^{r}$ e dimensão $p$ de $M$. Consideremos uma carta local $(U, \varphi) \in \mathcal{F}$ tal que $\varphi(U)=U_{1} \times U_{2} \subset \mathbb{R}^{p} \times \mathbb{R}^{n-p}$. Os conjuntos de forma $\varphi^{-1}\left(U_{1} \times\{c\}\right)$, $c \in U_{2}$ são chamados placas de $U$. Fixado $c \in U_{2}$, a aplicação $f=\left.\varphi^{-1}\right|_{U_{1} \times\{c\}}: U_{1} \times\{c\} \rightarrow$ $U$ é um mergulho de classe $C^{r}$, por tanto as placas são subvariedades conexas de dimensão $p$ e classe $C^{r}$. Além disto se $\alpha$ e $\beta$ são placas de $U$ então $\alpha \cap \beta=\emptyset$ ou $\alpha=\beta$.

Um caminho de placas de $\mathcal{F}$ é uma sequencia $\alpha_{1}, \cdots, \alpha_{k}$ de placas de $\mathcal{F}$ tal que $\alpha_{j} \cap \alpha_{j+1} \neq \emptyset$ para todo $j \in\{1, \cdots, k-1\}$. Como $M$ é recoberta pelas placas de $\mathcal{F}$, 
podemos definir em $M$ a seguinte relação de equivalência: $p \sim q$ se existe um caminho de placas $\alpha_{1}, \cdots, \alpha_{k} \operatorname{com} p \in \alpha_{1}$ e $q \in \alpha_{k}$. As classes de equivalência da relação " $\sim$ "são chamadas folhas de $\mathcal{F}$.

Da definição segue que uma folha $L$ de $\mathcal{F}$ é um subconjunto de $M$ conexo por caminhos. Outro fato importante é que toda folha $L$ de $\mathcal{F}$ possui uma estrutura de variedade $C^{r}$ de dimensão $p$ imersa em $M$ naturalmente induzida pelas cartas de $\mathcal{F}$. Em geral as folhas não são subvariedades de $M$.

Definição 1.2: Seja $\Sigma$ uma subvariedade de $M$. Dizemos que $\Sigma$ é transversal a $\mathcal{F}$ quando $\Sigma$ é transversal a todas as folhas de $\mathcal{F}$ que ela encontra. Quando $\operatorname{dim}(\Sigma)+\operatorname{dim}(\mathcal{F})=$ $\operatorname{dim}(M)$, dizemos que $\Sigma$ é uma seção transversal a $\mathcal{F}$.

Dado $p \in M$ sempre existe uma seção transversal de $\mathcal{F}$ passando por $p$. Com efeito basta considerarmos uma carta local $(U, \varphi) \in \mathcal{F}$ como $p \in U, \varphi(U)=U_{1} \times U_{2} \subset \mathbb{R}^{p} \times \mathbb{R}^{n-p}$, $\varphi(p)=\left(c_{1}, c_{2}\right)$ e tomarmos $\Sigma=\varphi^{-1}\left(\left\{c_{1}\right\} \times U_{2}\right)$. Como $\left\{c_{1}\right\} \times U_{2}$ é transversal às placas $U_{1} \times c, c \in U_{2}$, é claro que $\Sigma$ é seção transversal de $\mathcal{F}$.

Teorema 1.3 (Uniformidade transversal de $\mathcal{F}$ ): Seja L uma folha de $\mathcal{F}$. Dados $q_{1}, q_{2} \in$ $L$, existem seções transversais de $\mathcal{F}, \Sigma_{1}$ e $\Sigma_{2}$ com $q_{i} \in \Sigma_{i}(i=1,2)$ e um difeomorfismo $C^{r}$, $f: \Sigma_{1} \rightarrow \Sigma_{2}$ tal que para toda folha $L^{\prime}$ de $\mathcal{F}$ tem-se $f\left(L^{\prime} \cap \Sigma_{1}\right)=L^{\prime} \cap \Sigma_{2}$.

Observação 1.4: Da prova do Teorema anterior(ver $[6, p .49])$ segue que. Se $(U, \varphi)$ e $(V, \psi)$ são cartas locais de $\mathcal{F}$ tal que $U \cap V \neq \emptyset$ e $\varphi(U)=U_{1} \times U_{2} \subset \mathbb{R}^{p} \times \mathbb{R}^{n-p}$ e $\psi(V)=V_{1} \times V_{2} \subset \mathbb{R}^{p} \times \mathbb{R}^{n-p}$. Sejam $P$ placa em $U$ e $Q$ placa em $V$ talque $P \cap Q \neq \emptyset$. Se $p \in P, q \in Q ; \varphi(p)=\left(c_{1}, c_{2}\right), \psi(p)=\left(d_{1}, d_{2}\right)$ e consideramos as seções transversais $\Sigma=\varphi^{-1}\left(\left\{c_{1}\right\} \times U_{2}\right)$ e $\Sigma^{\prime}=\psi^{-1}\left(\left\{d_{1}\right\} \times V_{2}\right)$, então existe um difeomorfismo entre partes de $\Sigma$ e $\Sigma^{\prime}$ ao longo das folhas de $\mathcal{F}$.

Teorema 1.5: Seja L uma folha de uma folheação $\mathcal{F}$ de $M$. As afirmações abaixo são equivalentes:

a) L é folha fechada.

b) $S e(U, \varphi)$ é uma carta trivializadora de $\mathcal{F}$ tal que $\bar{U}$ é compacto então $U \cap L$ contém um número finito de placas de $U$.

A prova pode ser encontrada em [6]. 


\subsection{Espaços fibrados}

Os resultados de esta seção serão unicamente usados na demostração do Teorema 2.8.

Um espaço fibrado $(E, B, F, \pi)$, consiste de variedades diferenciáveis $E, B, F$ e de uma aplicação diferenciável $\pi: E \rightarrow B$. Além disso $E$ possui uma estrutura de produto local definida por uma cobertura $\left\{V_{i}\right\}_{i \in J}$ de $B$ e por difeomorfismos $\phi_{i}: \pi^{-1}\left(V_{i}\right) \rightarrow V_{i} \times F$, $i \in J$, que torna o seguente diagrama comutativo

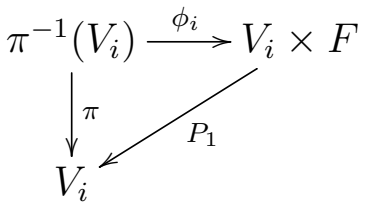

esto é, $\pi=P_{1} \circ \phi_{i}$, onde $P_{i}$ é a projeção na primeira coordenada. Em particular, $\pi$ é uma submersão sobre $B$ e $E$ é localmente difeomorfo ao produto de um aberto em $B$ por $F$.

O espaço $E$ é chamado espaço total, $B$ a base e $F$ a fibra. A aplicação $\pi$ é chamada projeção. Se $x \in B$, a subvariedade $F_{x}=\pi^{-1}(x) \simeq F$ é chamada fibra de $E$ sobre $x$. Se $\pi^{-1}(x)$ tem estrutura de espaço vetorial dizemos que é fibrado vetorial.

Definição 1.6: Sejam $\left\{V_{i}\right\}_{i \in J}$ uma cobertura aberta de uma variedade $B$ e $(G, \bullet)$ um grupo de Lie. Uma família de funções $C^{\infty}, \delta_{i j}: V_{i} \cap V_{j} \rightarrow G$ satisfazendo a condição

$$
\delta_{i k}(x)=\delta_{i j}(x) \bullet \delta_{j k}(x) \forall x \in V_{i} \cap V_{j} \cap V_{k}
$$

é chamada um cociclo de $B$ com valores no grupo $G$.

Lembremos que uma ação de um grupo topológico $G$ sobre uma variedade $F$ é um homomorfismo

tal que a aplicação

$$
\begin{aligned}
& \rho: G \rightarrow \operatorname{Diff}^{\infty}(F) \\
& \begin{aligned}
G \times F & \longrightarrow F \\
(g, x) & \mapsto \rho(g)(x)
\end{aligned} \text { é } C^{\infty}
\end{aligned}
$$

Definição 1.7: Dizemos que um espaço fibrado $(E, B, F, \pi)$ tem grupo estrutural $G(G$ grupo topológico), se $G$ age pela esquerda em $F$ e existe um cociclo de $B$ com valores em $G$ com respeito à cobertura $\left\{V_{i}\right\}_{i \in J}$ de $B$ que trivializa o fibrado tal que o difeomorfismo

$$
\phi_{i} \circ \phi_{j}^{-1}:\left(V_{i} \cap V_{j}\right) \times F \longrightarrow\left(V_{i} \cap V_{j}\right) \times F
$$

satisfaz 


$$
\phi_{i} \circ \phi_{j}^{-1}(x, y)=\left(x,\left(\rho \delta_{i j}(x)\right) y\right), \forall(x, y) \in\left(V_{i} \cap V_{j}\right) \times F
$$

Observação 1.8: É claro que dado um fibrado $(E, B, F, \pi)$ algumas vezes pode-se dotar com distintos tipos de grupos estruturais.

Exemplo 1.9: Seja $M^{m}$ uma variedade

i) O fibrado tangente de uma variedade $\left(T M, M, \mathbb{R}^{m}, \pi\right)$, onde

$$
T M=\left\{(x, v): x \in M, v \in T_{x} M\right\}
$$

e a projeção é definida

$$
\begin{aligned}
\pi: T M & \longrightarrow M \\
(x, v) & \mapsto x
\end{aligned}
$$

Tem grupo estrutural $G L(m, \mathbb{R})$.

ii) O fibrado normal de uma variedade $\left(\mathcal{V}(N), N, \mathbb{R}^{m-n}, \pi\right)$, onde $N^{n}$ é uma subvariedade de $M$,

$$
\mathcal{V}(N)=\left\{\left(p, v_{p}\right): p \in N, v_{p} \in T_{p} N^{\perp}\right\}
$$

e a projeção é

$$
\begin{aligned}
\pi: \mathcal{V}(N) & \longrightarrow N \\
\left(p, v_{p}\right) & \mapsto p
\end{aligned}
$$

é um fibrado vetorial que tem grupo estrutural $G L(m-n, \mathbb{R})$.

Definição 1.10: Um fibrado vetorial de posto $n$ é um fibrado com fibras $\mathbb{R}^{n}$ e grupo estrutural $G L(n, \mathbb{R})$.

Proposição 1.11: $O$ grupo estrutural de um fibrado vetorial real de posto $n$ pode sempre ser reduzido para as matrizes ortogonais reais $\mathcal{O}(n)$.

Demonstração. $\operatorname{Ver}([1, p .64])$

Teorema 1.12 (Teorema da vizinhança tubular): Seja $N \subset M$ uma subvariedade $C^{r}(r \geq 1)$. Existem uma vizinhança aberta $T(N) \supset N$ e uma submersão $C^{r} \pi: T(N) \rightarrow$ $N$, tais que $\pi(q)=q$ para todo $q \in N$. Se a codimensão de $N$ é $k$, então $T(N)$ pode ser obtida de tal forma $\left(T(N), N, \mathbb{R}^{k}, \pi\right)$ seja um espaço fibrado.

Demonstração. $\operatorname{Ver}([13])$ 
Definição 1.13: Sejam os fibrados $\xi_{i}=\left(E_{i}, B, F_{i}, \pi_{i}\right), i=1,2$. Dizemos que $\xi_{1}$ é equivalente a $\xi_{2}$ se existe um difeomorfismo $h: E_{1} \rightarrow E_{2}$ tal que cada fibra $\pi_{1}^{-1}(p)$ é levado de forma difeomorfo em $\pi_{2}^{-1}(p)$.

Teorema 1.14: Seja $N \subset M$ uma subvariedade compacta de $M$. Então $N$ tem uma vizinhança tubular $\pi: T(N) \rightarrow N$ em $M$ que é equivalente ao fibrado normal de $N$ em $M$ Demonstração. Ver $([22, p .468])$

\subsection{Dinâmica hiperbólica}

Nesta seção faremos um breve recorrido por alguns resultados principais desta teoria, para um estudo detalhado e ver as provas dos resultados que são enunciados nesta seção, se recomenda os livros [12], [21] e [18]. Daqui em diante $M$ denotará uma variedade riemanniana compacta, conexa e sem bordo.

Definição 1.15 (Algumas Propiedades Topologicas): Seja $f: M \rightarrow M$ um difeomorfismo.

a) Dizemos que $f$ é transitivo se dados $U, V \subset M$ abertos quaisquer, existe $m>0$ tal que $f^{-m}(U) \cap V \neq \emptyset$.

b) Dizemos que $f$ é topologicamente mixing se dados $U, V \subset M$ abertos quaisquer, existe $m>0$ tal que $f^{-n}(U) \cap V \neq \emptyset \forall n \geq m$.

c) Dizemos que $f$ é expansivo se existe $\alpha>0$ tal que se $d\left(f^{n}(x), f^{n}(y)\right)<\alpha \forall n \in \mathbb{Z}$ então $x=y$ ( $\alpha$ é chamado constante de expansividade).

Definição 1.16: Seja $f: M \rightarrow M$ um difeomorfismo. Um conjunto compacto e invariante $\Lambda$ se diz que é hiperbólico se para cada $x \in \Lambda$ existem subespaços $E^{s}(x) \subset T_{x} M$ e $E^{u}(x) \subset T_{x} M$ que verificam:

i) $T_{x} M=E^{s}(x) \oplus E^{u}(x)$.

ii) $D f_{x}\left(E^{s}(x)\right)=E^{s}(f(x))$ e $D f_{x}\left(E^{u}(x)\right)=E^{u}(f(x))$.

iii) Existem contantes $c>0$ e $\lambda \in(0,1)$ tal que

a) $\left\|D f_{x}^{n}(v)\right\| \leq c \lambda^{n}\|v\|, \forall v \in E^{s}(x)$ e $n \geq 0$.

b) $\left\|D f_{x}^{-n}(v)\right\| \leq c \lambda^{n}\|v\|, \forall v \in E^{u}(x)$ e $n \geq 0$. 
Definição 1.17: Seja $f: M \rightarrow M$ um difeomorfismo e $x \in M$. Se define o conjunto estável de $x$ como

$$
W^{s}(x)=\left\{y \in M: d\left(f^{n}(x), f^{n}(y)\right) \longrightarrow_{n \rightarrow+\infty} 0\right\}
$$

e o instável como

$$
W^{u}(x)=\left\{y \in M: d\left(f^{n}(x), f^{n}(y)\right) \longrightarrow_{n \rightarrow-\infty} 0\right\}
$$

Para $\epsilon>0$ definimos o conjunto estável e instável local(de tamanho $\epsilon$ ) como

$$
\begin{gathered}
W_{\epsilon}^{s}(x)=\left\{y \in M: d\left(f^{n}(x), f^{n}(y)\right)<\epsilon, \forall n \geq 0\right\} \\
W_{\epsilon}^{u}(x)=\left\{y \in M: d\left(f^{-n}(x), f^{-n}(y)\right)<\epsilon, \forall n \geq 0\right\}
\end{gathered}
$$

Observação 1.18: $f\left(W_{\epsilon}^{s}(x)\right) \subset W_{\epsilon}^{s}(f(x))$ e $f\left(W_{\epsilon}^{u}(x)\right) \supset W_{\epsilon}^{u}(f(x))$. De fato, seja $z \in f\left(W_{\epsilon}^{s}(x)\right)$, existe $y \in W_{\epsilon}^{s}(x)$ tal que $z=f(y)$ e $d\left(f^{n}(x), f^{n}(y)\right)<\epsilon, \forall n \geq 0$. Logo, se $m=n-1$ temos que,

$$
d\left(f^{m}(f(x)), f^{m}(\underbrace{f(y)}_{z})\right)<\epsilon, \forall m \geq 0
$$

por tanto $z \in W_{\epsilon}^{s}(f(x))$. De forma análoga se mostra $f\left(W_{\epsilon}^{u}(x)\right) \supset W_{\epsilon}^{u}(f(x))$.

Teorema 1.19 (Teorema da variedade estável): Seja $f: M \rightarrow M$ um difeomorfismo $C^{r}$ e $\Lambda$ um conjunto hiperbólico. Então existe $\epsilon>0$ tal que para quaisquer $x \in \Lambda$ se verifica que:

i) $W_{\epsilon}^{s}(x)$ e $W_{\epsilon}^{u}(x)$ são subvariedades mergulhadas de classe $C^{r} \operatorname{com} T_{x} W_{\epsilon}^{s}(x)=E^{s}(x)$ $e T_{x} W_{\epsilon}^{u}(x)=E^{u}(x)$.

ii) $d\left(f^{n}(x), f^{n}(y)\right)<\lambda^{n} d(x, y)$ para $y \in W_{\epsilon}^{s}(x), n \geq 0 e$ $d\left(f^{-n}(x), f^{-n}(y)\right)<\lambda^{n} d(x, y)$ para $y \in W_{\epsilon}^{u}(x), n \geq 0$.

iii) $W^{s}(x)=\cup_{n \geq 0} f^{-n}\left(W_{\epsilon}^{s}\left(f^{n}(x)\right)\right)$ e $W^{u}(x)=\cup_{n \geq 0} f^{n}\left(W_{\epsilon}^{u}\left(f^{-n}(x)\right)\right)$. $W^{s}(x)$ e $W^{u}(x)$ são subvariedades imersas de classe $C^{r}$ que variam continuamente(como subvariedades $C^{r}$ y em subconjuntos compactos) com $x$.

Observação 1.20: Do teorema anterior, se deduz que existe $\delta>0$ se $x, y \in \Lambda$ com $d(x, y)<\delta$ então $W_{\epsilon}^{s}(x)$ e $W_{\epsilon}^{u}(y)$ se intersectam transversalmente num único ponto.

Corolário 1.21: Seja $f: M \rightarrow M$ um difeomorfismo $C^{r}$ e $\Lambda$ um conjunto hiperbólico. Então $\left.f\right|_{\Lambda}$ é expansivo. 
Definição 1.22: Seja $f: M \rightarrow M$ um difeomorfismo $C^{r}$ e $\Lambda$ um conjunto hiperbólico. Dizemos que $\Lambda$ tem estrutura de produto local se existe $\delta>0$ tal que se $x, y \in \Lambda$ com $d(x, y)<\delta$ então $W_{\epsilon}^{s}(x) \cap W_{\epsilon}^{u}(y) \in \Lambda$.

O importante da definição anterior é que o ponto de interseção pertença a $\Lambda$, já que pela observação anterior sabemos que variedades estáveis e instáveis de pontos cercanos se cortam transversalmente. A definição diz que efetivamente $\Lambda$ localmente é um produto:

$$
\Lambda \cap B(x, \delta) \approx W_{\epsilon}^{s}(x) \cap \Lambda \times W_{\epsilon}^{u}(x) \cap \Lambda .
$$

Proposição 1.23: Seja $f: M \rightarrow M$ um difeomorfismo e suponhamos que $\overline{\operatorname{Per}(f)}$ é um conjunto hiperbólico. Então $\overline{\operatorname{Per}(f)}$ tem estrutura de produto local.

Proposição 1.24: Seja $f: M \rightarrow M$ um difeomorfismo $C^{r}$ e $\Lambda$ um conjunto hiperbólico com produto local. Então a aplicação

$$
[\cdot, \cdot]: \Lambda \times \Lambda \rightarrow \Lambda
$$

dado por $[x, y]=W_{\epsilon}^{s}(x) \cap W_{\epsilon}^{u}(y)$ é continua.

Definição 1.25: Sejam $f: M \rightarrow M$ e $g: N \rightarrow N$ dois transformações continuas em espaços topológicos compactos. Dizemos que $g$ é um fator topológico de $f$ se existe uma aplicação contínua e sobrejetiva $\phi: M \rightarrow N$ tal que $\phi \circ f=g \circ \phi$. $\phi$ é chamado semi-conjugação de $f$ e $g$.

Definição 1.26: Sejam $f: M \rightarrow M$ e $g: N \rightarrow N$ dois difeomorfismos. Dizemos que $f$ e $g$ são topologicamente conjugadas se existe $\pi: M \rightarrow N$ homeomorfismo tal que $\pi \circ f=g \circ \pi$.

Proposição 1.27: Se $f: M \rightarrow M$ e $g: N \rightarrow N$ são topologicamente conjugados pelo homeomorfismo $\pi: M \rightarrow N$. Então,

i) $x$ é um ponto periódico de $f$ se, e somente se, $\pi(x)$ é ponto periódico de $g$.

ii) $f$ é topologicamente mixing se, e somente se, g é topologicamente mixing.

\subsection{Partições de Markov}

Nesta seção definimos o que é uma partição de Markov e enunciamos alguns resultados desta teoria que precisaremos ao longo deste trabalho, para ver as provas e fazer um 
estudo detalhado se recomenda o livro [2]. No capítulo 4, apresentamos explicitamente as partições de Markov dos sistemas dinâmicos Thom-Arnold map e da ferradura de Smale. Seja $f: M \rightarrow M$ um difeomorfismo $C^{r}$ e $\Lambda$ um conjunto hiperbólico com estrutura de produto local. Seja $\epsilon>0$ como no teorema 1.19 e $\delta>0$ como na observação 1.20.

Definição 1.28: Dizemos que um conjunto $R \subset \Lambda$ não vazio é um retângulo para $\left.f\right|_{\Lambda}$ se tem diâmetro menor que $\min \{\delta, \epsilon\}$ e

$$
[x, y]=W_{\epsilon}^{s}(x) \cap W_{\epsilon}^{u}(x) \in R
$$

para todo $x$ e $y$ em $R$. O retângulo é chamado próprio se $R$ é fechado e $R=\overline{\operatorname{int}(R)}$. Para $x \in R$ definimos os conjuntos

$$
\begin{aligned}
& W^{s}(x, R)=W_{\epsilon}^{s}(x) \cap R \\
& W^{u}(x, R)=W_{\epsilon}^{u}(x) \cap R
\end{aligned}
$$

Proposição 1.29: Sejam $x, y \in R, R$ retângulo. Então

$$
y \in W^{s}(x, R) \Leftrightarrow W^{s}(x, R)=W^{s}(y, R)
$$

analogamente

$$
y \in W^{u}(x, R) \Leftrightarrow W^{u}(x, R)=W^{u}(y, R)
$$

Definição 1.30: Se chama bordo estável de um retângulo $R$ ao conjunto

$$
\partial^{s}(R)=\left\{x \in R: x \notin \operatorname{int} W^{u}(x, R) \operatorname{em} W_{\epsilon}^{u}(x) \cap \Lambda\right\}
$$

Se chama bordo instável de um retângulo $R$ ao conjunto

$$
\partial^{u}(R)=\left\{x \in R: x \notin \operatorname{int} W^{s}(x, R) \operatorname{em} W_{\epsilon}^{s}(x) \cap \Lambda\right\}
$$

Para justificar o nome de bordo estável, demostraremos que $\partial^{s}(R)$ esta formado pela união de $\epsilon$-variedades estáveis em $R$. Analogamente tem-se com $\partial^{u}(R)$

Proposição 1.31: $y \in \partial^{s} R$ implica $W^{s}(y, R) \subset \partial^{s} R$. analogamente $y \in \partial^{u} R$ implica $W^{u}(y, R) \subset \partial^{u} R$.

A proposição seguente mostra que para retângulos $R$ fechados, o bordo topológico de $R$ é a união do bordo estável e instável. .

Proposição 1.32: Se $R$ é um retângulo fechado então $\partial R=\partial^{s} R \cup \partial^{u} R$. 
Definição 1.33: Uma partição de Markov de $\Lambda$ é uma cobertura finita $\mathcal{R}=\left\{R_{1}, \cdots, R_{m}\right\}$ de $\Lambda$ por retângulos próprios fechados tais que:

i) $\operatorname{int} R_{i} \cap \operatorname{int} R_{j}=\emptyset$, para $i \neq j$.

ii) Se $x \in \operatorname{int} R_{i} \cap f^{-1}\left(\operatorname{int} R_{j}\right)$ então

a) $f\left(W^{s}\left(x, R_{i}\right)\right) \subset W^{s}\left(f(x), R_{j}\right)$,

b) $f\left(W^{u}\left(x, R_{i}\right)\right) \supset W^{u}\left(f(x), R_{j}\right)$.

Teorema 1.34: Seja $f: M \rightarrow M$ um difeomorfismo e $\Lambda$ um conjunto hiperbólico com estrutura de produto local. Se $\left.f\right|_{\Lambda}$ é transitivo, então $\Lambda$ possui uma partição de Markov.

Definição 1.35: Seja $\mathcal{R}=\left\{R_{1}, \cdots, R_{m}\right\}$ uma partição de Markov. Se chama bordo de $\mathcal{R}$ ao conjunto

$$
\partial \mathcal{R}=\cup_{j=1}^{m} \partial R_{j}
$$

Se chama bordo estável de $\mathcal{R}$ a

$$
\partial^{s} \mathcal{R}=\cup_{j=1}^{m} \partial^{s} R_{j}
$$

e se chama bordo instável de $\mathcal{R}$ a

$$
\partial^{u} \mathcal{R}=\cup_{j=1}^{m} \partial^{u} R_{j}
$$

Se observa da Proposição (1.32) que

$$
\partial \mathcal{R}=\partial^{s} \mathcal{R} \cup \partial^{u} \mathcal{R}
$$

Proposição 1.36: Seja $\mathcal{R}=\left\{R_{1}, \cdots, R_{m}\right\}$ uma partição de Markov. Então

1) $\partial \mathcal{R}$ tem interior vazio e é fechado.

2) $\cup_{j=1}^{m}$ intR $R_{j}$ é aberto e denso em $\Lambda$

3) $\Lambda \backslash \cup_{n \in \mathbb{Z}} f^{-n}(\partial \mathcal{R})$ é denso em $\Lambda$ e invariante por $f$.

A seguinte proposição que será fortemente utilizado no Capítulo 5, permite aplicar as condições (a) e (b) da definição de partição de Markov a outros pontos $x \in \Lambda$ que não estão necessariamente em $\operatorname{int} R_{i} \cap f^{-1}\left(\operatorname{int} R_{j}\right)$.

Proposição 1.37: Seja $\mathcal{R}=\left\{R_{1}, \cdots, R_{m}\right\}$ uma partição de Markov de $\Lambda$. Se int $R_{i} \cap$ $f^{-1}\left(\right.$ int $\left.R_{j}\right) \neq \emptyset$, então para todo $y \in R_{i} \cap f^{-1}\left(R_{j}\right)$ tem-se: 
i) $f\left(W^{s}\left(x, R_{i}\right)\right) \subset W^{s}\left(f(x), R_{j}\right)$,

ii) $f\left(W^{u}\left(x, R_{i}\right)\right) \supset W^{u}\left(f(x), R_{j}\right)$.

Proposição 1.38: Seja $\mathcal{R}=\left\{R_{1}, \cdots, R_{m}\right\}$ uma partição de Markov de $\Lambda$ então

$$
f\left(\partial^{s} \mathcal{R}\right) \subset \partial^{s} \mathcal{R} \text { e } f\left(\partial^{u} \mathcal{R}\right) \supset \partial^{u} \mathcal{R}
$$

\subsection{Entropia}

A palavra entropia foi inventada em 1865 pelo físico e matemático alemão Rudolf Clausius um dos primeiros fundadores da termodinâmica. A entropia pode interpreta-se como uma medida do grau de "desordem"de um sistema. Se diz que um sistema altamente distribuído ao azar tem entropia alta. Nesta seção apresentaremos alguns resultados desta teoria que precisaremos ao longo deste trabalho, para um estudo detalhado o autor recomenda os livros [16] e [14]. Na primeira sub-seção definimos a entropia de uma transformação relativa a uma probabilidade invariante que foi proposto por KolmogorovSinai. Na seguente sub-seção definimos a entropia topológica que foi proposto por R. Alder, A. Konhein e McAndrew, inspirados na entropia de Kolmogorov-Sinai, esta noção se aplica a qualquer transformação continua num espaço topológico compacto. Logo enunciamos o Teorema de Principio Variacional que faz a ligação dos dois conceitos. No capítulo 5, calculamos as entropias de Thom-Arnold map e a ferradura de Smale.

\subsubsection{Entropia com respeito a uma medida}

Seja $(M, \mathcal{B}, \mu)$ um espaço de probabilidade. Neste capítulo, por partição sempre entenderemos uma família finita ou enumerável $\mathcal{P}$ de conjuntos mensuráveis disjuntos doisa-dois e cuja união tem medida total. A soma $\mathcal{P} \vee \mathcal{Q}$ de duas partições $\mathcal{P}$ e $\mathcal{Q}$ é a partição cujos elementos são as interseções $P \cap Q \operatorname{com} P \in \mathcal{P}$ e $Q \in \mathcal{Q}$. Mais geralmente, dada qualquer família enumerável de partições $\mathcal{P}_{n}$, definimos

$$
\bigvee_{n} \mathcal{P}_{n}=\left\{\bigcap_{n} P_{n}: P_{n} \in \mathcal{P}_{n} \text { para cada } n\right\}
$$

Definição 1.39: Seja $\mathcal{P}$ uma partição finita do espaço de probabilidade $(M, \mathcal{B}, \mu)$. Seja $\phi(x)=x \log x$ se $0<x \leq 1$ e $\phi(0)=0$.

Definimos a entropia de $\mathcal{P}$ como

$$
H(\mathcal{P})=-\sum_{P \in \mathcal{P}} \phi(\mu(P))
$$


Seja $f: M \rightarrow M$ uma transformação que preserva medida e seja $\mathcal{P}$ uma partição de $M$, então $f^{-j} \mathcal{P}=\left\{f^{-j}(P): P \in \mathcal{P}\right\}$ é também uma partição de $M$.

Definição 1.40: Suponhamos que $f: M \rightarrow M$ que preserva medida e seja $\mathcal{P}$ uma partição, definimos a entropia $h(f, \mathcal{P})$ de $f$ respeito a $\mathcal{P}$ como

$$
h(f, \mathcal{P})=\lim _{n \rightarrow \infty} \frac{1}{n} H\left(\bigvee_{j=0}^{n-1} f^{-j}(\mathcal{P})\right)
$$

Definição 1.41: A entropia de uma transformação $f:(M, \mathcal{B}, \mu) \rightarrow(M, \mathcal{B}, \mu)$ que preserva medida se define como

$$
h_{\mu}(f)=\sup \{h(f, \mathcal{P}): \mathcal{P} \text { é partição finita }\}
$$

Definição 1.42: Sejam $\mu$ e $\nu$ medidas de probabilidade invariantes por transformações $f: M \rightarrow M$ e $g: N \rightarrow N$, respectivamente. Dizemos que os sistemas $(f, \mu)$ e $(g, \nu)$ são ergodicamente equivalentes se podemos escolher conjuntos mensuráveis $X \subset M$ e $Y \subset N$ com $\mu(X)=1$ e $\nu(Y)=1$, e uma bijeção mensurável $\phi: X \rightarrow Y$ com inversa mensurável, de tal forma que

$$
\phi_{*} \mu=\nu \quad \text { e } \quad \phi \circ f=g \circ \phi .
$$

Proposição 1.43: Sejam $f: M \rightarrow M$ e $g: N \rightarrow N$ transformações preservando probabilidades $\mu$ em $M$ e $\nu$ em $N$. Se $(f, \mu)$ é ergodicamente equivalente a $(g, \nu)$, então

$$
h_{\mu}(f)=h_{\nu}(g) .
$$

\subsubsection{Entropia topológica}

Seja $M$ um espaço topológico compacto. Chamamos cobertura aberta de $M$ qualquer família $\mathcal{U}$ de abertos cuja união é todo o $M$. Dadas as coberturas abertas $\mathcal{U}_{1}, \cdots, \mathcal{U}_{m}$ de $M$ denotamos por $\mathcal{U}_{1} \vee \cdots \vee \mathcal{U}_{m}$ a sua soma, isto é, a cobertura aberta cujos elementos são as interseções $A_{1} \cap \cdots \cap A_{m} \operatorname{com} A_{j} \in \mathcal{U}_{j}$ para cada $j$.

Seja $f: M \rightarrow M$ uma transformação contínua. Se $\mathcal{U}$ uma cobertura aberta de $M$, então $f^{-j}(\mathcal{U})=\left\{f^{-j}(A): A \in \mathcal{U}\right\}$ também é uma cobertura. Para cada $n \geq 1$, denotamos

$$
\mathcal{U}^{n}=\mathcal{U} \vee f^{-1}(\mathcal{U}) \vee \cdots \vee f^{n-1}(\mathcal{U})
$$

Definição 1.44: Seja $M$ um espaço topológico compacto. Seja $\mathcal{U}$ uma cobertura aberta de $M$, e $f: M \rightarrow M$ uma transformação continua. 
a) Definimos a entropia de $\mathcal{U}$ por

$$
H(\mathcal{U})=\log N(\mathcal{U})
$$

onde $N(\mathcal{U})$ denota a mínima cardinalidade de uma subcobertura finita de $\mathcal{U}$.

b) Definimos a entropia de $f$ com respeito $\mathcal{U}$

$$
h(f, \mathcal{U})=\lim _{n \rightarrow \infty} \frac{1}{n} H\left(\mathcal{U}^{n}\right)
$$

Definição 1.45: Seja $M$ um espaço topológico compacto. Seja $\mathcal{U}$ uma cobertura aberta de $M$, e $f: M \rightarrow M$ uma transformação continua, definimos a entropia topologia de $f$ como

$$
h_{\text {top }}(f)=\sup \{h(f, \mathcal{U}): \mathcal{U} \text { é uma cobertura aberta de } M\}
$$

Observação 1.46: A entropia topológica é usualmente definida por meio de conjuntos $(n, \epsilon)$-separador, esta definicao é equivalente à definição 1.45 e foi proposta por Efim Dinaburg e Rufus Bowen. Neste caso a entropia topológica é a taxa de crescimento exponencial do número de orbitas que são distinguíveis dentro de um certo grau de precisão arbitrariamente pequeno.

Proposição 1.47: Se $T_{2}: X_{2} \rightarrow X_{2}$ é um fator topológico de $T_{1}: X_{1} \rightarrow X_{1}$ então

$$
h_{\text {top }}\left(T_{2}\right) \leq h_{\text {top }}\left(T_{1}\right)
$$

O seguente teorema é um dos teoremas importantes da teoria ergódica, ele relaciona o dois conceitos de entropia.

Teorema 1.48 (Principio Variacional): Sejam $X$ um espaço métrico compacto, $T: X \rightarrow$ $X$ continua. Então

$$
h_{\text {top }}(T)=\sup \left\{h_{\mu}(T): \mu \in \mathcal{M}_{T}(X)\right\}
$$

Um problema antigo e muito importante é em saber se o supremo é um máximo, e neste caso se o máximo é alcançado em uma única medida de probabilidade ou não. R. Bowen, dá condições suficientes para dar uma resposta positiva a esta questão. De fato o R. Bowen [3] mostra que se $X$ é um espaço métrico compacto e $f: X \rightarrow X$ um homeomorfismo expansivo com a propriedade de especificação, existe uma única medida de probabilidade $f$-invariante que maximiza a entropia métrica. No capitulo 5, apresentaremos a prova deste resultado, também devido R. Bowen [4], [5], para difeomorfismos topologicamente mixing(ver Teorema 5.4). 


\subsection{Medida de Markov}

Nesta seção definimos a medida de Markov e enunciamos alguns resultados que precisaremos, para ver as provas dos resultados enunciados e fazer um profundo estudo desta seção se recomenda os livros [14] e [16].

Seja $\Sigma=\{1, \cdots, n\}^{\mathbb{Z}}$ e $\sigma: \Sigma \rightarrow \Sigma$ a transformação de deslocamento à esquerda. A dinâmica de este sistema $(\Sigma, \sigma)$ é estudado na seção 4.2 do capitulo 4 .

Definição 1.49: Uma matriz $P=\left(P_{i j}\right)$ é estocástica se os coeficientes $P_{i j}$ satisfazem:

i) a) $P_{i j} \geq 01 \leq i, j \leq n$

b) $\sum_{j=1}^{n} P_{i j}=1,1 \leq i \leq n$.

ii) Existe um vetor $p=\left(p_{1}, \cdots, p_{n}\right)$, chamado vetor de probabilidade tal que

a) $p_{j}>01 \leq j \leq n$

b) $\sum_{j=1}^{n} p_{j}=1$

c) $\sum_{i=1}^{n} p_{i} P_{i j}=p_{j}$

Seja $P=\left(P_{i j}\right)$ uma matriz estocástica e $p=\left(p_{i}\right)_{i}$ um vetor de probabilidade. Definimos a seguinte medida $\mu$ sobre os conjuntos de Borel de $\Sigma$ por

$$
\mu\left(\left[m, \alpha_{m}, \cdots, \alpha_{l}\right]\right)=p_{\alpha_{m}} P_{\alpha_{m} \alpha_{m+1}} \cdots P_{\alpha_{l-1} \alpha_{l}}
$$

onde $\left[m, \alpha_{m}, \cdots, \alpha_{l}\right]=\left\{\theta \in \Sigma: \theta_{m}=\alpha, \cdots, \theta_{l}=\alpha_{l}\right\}$ é um cilindro.

A medida $\mu$ é chamada medida de Markov associada a la matriz estocástica $P=\left(P_{i j}\right)$ e ao vetor de probabilidade $p=\left(p_{i}\right)_{i}$.

\section{Observação 1.50:}

i) A medida de Markov associada à matriz estocástica $P=\left(P_{i j}\right)$ e ao vetor de probabilidade $p=\left(p_{i}\right)_{i}$ é invariante pelo deslocamento $\sigma$.

ii) Esta medida é uma generalização das medidas de Bernoulli.

Da definição 1.49 - ii temos que $P^{*} p=p$, onde $P^{*}$ representa a transposta da matriz $P$. Em outras palavras, as medidas de Markov correspondem precisamente aos autovetores da matriz transposta para o autovalor 1 . O seguinte resultado clássico permite mostrar que tais autovetores sempre existem. 
Teorema 1.51 (Perron-Frobenius [15]): Seja B uma matriz $k \times k$ não negativa aperiódica (i.e., $B_{i j} \geq 0$ para cada $1 \leq i, j \leq k$ e existe $n>0$ tal que $B^{n}>0$ ). Então

i) Existe um autovalor $\lambda>0$ tal que $|\beta|<\lambda$ para todos os demais autovalores.

ii) $\lambda$ é de multiplicidade 1 .

iii) Existe um único autovetor à esquerda $u=\left(u_{1}, \cdots, u_{k}\right)$ tal que $u_{j}>0, \sum u_{j}=1$ e $u B=\lambda u$.

iv) Existe um único autovetor à direita $v=\left(v_{1}, \cdots, v_{k}\right)$ tal que $v_{j}>0, \sum v_{j}=1 e$ $B v=\lambda v$.

v) Autovetores correspondentes a autovalores distintos de $\lambda$ são não positivos; i.e., ao menos uma coordenada é positiva e ao menos uma coordenada é negativa.

Dada uma matriz $n \times n, A$ com coeficientes 0 ou 1 definimos o conjunto $\Sigma_{A}$ como: $\Sigma_{A}=\left\{\theta \in \Sigma: A_{\theta_{i} \theta_{i+1}}=1\right.$ para todo $\left.i \in \mathbb{Z}\right\}$ onde $\Sigma=\{1, \cdots, n\}^{\mathbb{Z}}$ e $A=(A i j)$.

Proposição 1.52: $\Sigma_{A}$ é compacto em $\Sigma$ e $\sigma\left(\Sigma_{A}\right) \subset \Sigma_{A}$.

Denotaremos por $\sigma: \Sigma_{A} \rightarrow \Sigma_{A}$ a restrição do deslocamento $\sigma$ a este compacto invariante. Ela é chamado do deslocamento de tipo finito.

Proposição 1.53: $\sigma: \Sigma_{A} \rightarrow \Sigma_{A}$ é topologicamente mixing se, e somente se, $A$ é aperiódica (i.e., existe $m>0$ tal que $A_{i j}^{m}>0$ onde $A^{m}=\left(A_{i j}^{m}\right)$ ).

Proposição 1.54: Seja P uma matriz estocástica e $p$ um vetor de probabilidade associado a P. O suporte da medida de Markov associado a $P$ e $p$ é $\Sigma_{A}$, onde $A$ é definido como $A_{i j}=1$ se $P_{i j}>0$ e $A_{i j}=0$ se $P_{i j}>0$.

Dado $P$ uma matriz estocástica e $p$ um vetor de probabilidade sempre podemos definir uma medida de Markov em $\Sigma$, então existem infinitas medidas de Markov em $\Sigma$ e em particular em $\Sigma_{A}$. Em 1966 Parry demonstrou que existe uma delas caracterizado pela propriedade de dar o máximo valor possível à entropia de $\sigma: \Sigma_{A} \rightarrow \Sigma_{A}$ e que descreve a forma em que os pontos periódicos de $\sigma$ se distribuem no espaço $\Sigma_{A}$.

Se $\sigma: \Sigma_{A} \rightarrow \Sigma_{A}$ é topologicamente mixing, então pela Proposição $1.53 A$ estocástica e pelo Teorema de Perron-Frobenius, existe $\lambda>0$ autovalor dominante de $A$. 
Teorema 1.55 (Parry [14]): Se $\sigma: \Sigma_{A} \rightarrow \Sigma_{A}$ é topologicamente mixing e $\lambda$ é o autovalor dominante de A então

$$
h_{\nu}(\sigma) \leq \log \lambda
$$

Para todo $\nu \in M_{\nu}\left(\Sigma_{A}\right)$ e existe uma única medida $\mu \in M_{\nu}\left(\Sigma_{A}\right)$ tal que

$$
h_{\mu}(\sigma)=\log \lambda=\lim _{n \rightarrow \infty} \frac{1}{n} \log \# \text { Fix }\left(\sigma^{n}\right)
$$

esta medida $\mu$ é uma medida de Markov positiva sobre abertos de $\Sigma_{A}$.

Observação 1.56: Pelo Teorema de Perron-Frobenius, existem únicos autovetores $u=$ $\left(u_{1}, \cdots, u_{n}\right)$ e $v=\left(v_{1}, \cdots, v_{n}\right)$ a esquerda e direita respectivamente associados a $\lambda$ tal que $\sum_{i} u_{i}=1=\sum_{i} v_{i}$. Definimos

$$
\begin{gathered}
P_{i j}=\frac{A_{i j} v_{j}}{\lambda v_{i}} \\
p_{i}=\frac{u_{i} v_{i}}{c}
\end{gathered}
$$

onde $c=\sum_{i=1}^{n} u_{i} v_{i}$. Parry prova que $P$ é uma matriz estocástica e $p$ um vetor de probabilidade associado a $P$; e que a única medida de Markov que satisfaz (1.2) é definia por $P$ e $p$. 


\section{$\longrightarrow$ \\ Medidas transversas invariantes por holonomia}

As medidas invariantes por um fluxo é um objeto de grande importância e utilidade para estudar a dinâmica dos fluxos. A generalização destas medidas em folheações é chamada medidas transversas invariantes por holonomia e é conseguida considerando medidas no espaço transverso à folheação em onde se identificam os pontos que pertencem a uma mesma folha e pedindo que sejam invariantes baixo o "fluxo das folhas". As medidas transversas têm sido objeto de estudo desde os anos 50 e demostraram ser muito úteis para a compreensão da dinâmica das folheações. O resultado principal deste capítulo, é mostrar um resultado conhecido de J. Plante [17] sobre a existência de medidas transversas invariantes sob a hipótese de crescimento sub-exponencial. Este capítulo é baseada no artigo de J. Plante [17].

\subsection{Medida transversa invariante}

Nesta seção $\mathcal{F}$ será uma folheação de dimensão $p$ de uma variedade $M$ de dimensão $n$ e classe $C^{\infty}$.

As propriedades da definição de folheação e a observação 1.3 podem ser resumidos como: Existe uma cobertura $\mathcal{U}=\left\{U_{\alpha}\right\}_{\alpha}$ de $M$ com as seguintes propriedades:

1. $\mathcal{U}$ é localmente finita.

2. Cada aberto $U_{\alpha}$ é conexo e $\left.\mathcal{F}\right|_{U_{\alpha}}$ é a folheação trivial; i.e., existem difeomorfismos $\varphi_{\alpha}: U_{\alpha} \rightarrow \varphi_{\alpha}\left(U_{\alpha}\right) \subset \mathbb{R}^{n}$ que levam a $\mathcal{F}$ na folheação horizontal de $\mathbb{R}^{p} \times \mathbb{R}^{n-p}=\mathbb{R}^{n}$. 
3. Estos difeomorfismos $\varphi_{\alpha}$ satisfazem que $\varphi_{\alpha}\left(U_{\alpha}\right) \subset \mathbb{R}^{n}$ e $\left\{\varphi_{\alpha}^{-1}\left((-1,1)^{n}\right)\right\}_{\alpha}$ é uma coberta de $M$.

4. Em cada $U_{\alpha}$ temos um disco imerso $\mathbb{D}^{n-p}=\Sigma_{\alpha} \subset \varphi_{\alpha}^{-1}\left((-1,1)^{n}\right)$, que é transversal às placas de $\left.\mathcal{F}\right|_{U_{\alpha}}$ e parametriza este espaço de placas.

5. Cada folha de $\mathcal{F}$ corta algum disco transversal $\Sigma_{\alpha}$ e se $U_{\alpha} \cap U_{\beta} \neq \emptyset$ segue que cada placa de $\left.\mathcal{F}\right|_{U_{\alpha}}$ encontra no máximo uma placa de $\left.\mathcal{F}\right|_{U_{\beta}}$ definindo um difeomorfismo $C^{\infty}, \gamma_{\alpha \beta}: D\left(\gamma_{\alpha \beta}\right) \subset \Sigma_{\alpha} \rightarrow R\left(\gamma_{\alpha \beta}\right) \subset \Sigma_{\beta}$ com a propriedade de que, em cada interseção $U_{\alpha} \cap U_{\beta} \neq \emptyset, \pi_{\beta}=\gamma_{\alpha \beta} \circ \pi_{\alpha} \operatorname{com} \pi_{\alpha}$ a projeção de $U_{\alpha}$ sobre $\Sigma_{\alpha}$ ao longo das placas.

A coberta $\mathcal{U}$ é chamado cobertura folheada regular de $\mathcal{F}$. Quando $\mathcal{U}$ é uma cobertura folheada regular temos que o conjunto $\Sigma=\bigsqcup_{\alpha} \Sigma_{\alpha}$, onde $\sqcup$ indica que a soma é disjunta, tem estrutura de variedade diferenciável de codimensão a dimensão de $\mathcal{F}$, esta variedade é chamada o espaço transverso de $\mathcal{F}$.

Observação 2.1: Os difeomorfismos $\gamma_{\alpha \beta}$ satisfazem a condição de cociclo; i.e., se $U_{\alpha} \cap U_{\beta} \neq \emptyset \Rightarrow \gamma_{\alpha \beta}=\gamma_{\beta \alpha}^{-1}$ e se $U_{\alpha} \cap U_{\beta} \cap U_{\delta} \neq \emptyset \Rightarrow \gamma_{\alpha \beta} \circ \gamma_{\beta \delta}=\gamma_{\alpha \delta}$ sobre os domínios correspondentes.

Agora lembremos a definição de pseudogrupo.

Definição 2.2: Seja $X$ um espaço topológico e seja $\Gamma$ uma coleção de homeomorfismos entre subconjuntos de $X$. Se $\gamma \in \Gamma$ denotamos por $D(\gamma)$ e $R(\gamma)$ ao domínio e ao imagem de $\gamma$, respectivamente. Dizemos que $\Gamma$ é um pseudogrupo se as seguentes condições satisfazem.

i) A identidade $i d: X \rightarrow X$ pertence a $\Gamma$.

ii) Se $\gamma \in \Gamma$ então $\gamma^{-1} \in \Gamma$, onde

$$
D\left(\gamma^{-1}\right)=R(\gamma) \text { e } R\left(\gamma^{-1}\right)=D(\gamma)
$$

iii) Se $\gamma_{1}, \gamma_{2} \in \Gamma$, então $\gamma_{1} \circ \gamma_{2} \in \Gamma$, onde

$$
D\left(\gamma_{1} \circ \gamma_{2}\right)=\gamma_{2}^{-1}\left(D\left(\gamma_{1}\right) \cap R\left(\gamma_{2}\right) \text { e } R\left(\gamma_{1} \circ \gamma_{2}\right)=\gamma_{1}\left(D\left(\gamma_{1}\right) \cap R\left(\gamma_{2}\right)\right)\right.
$$


iv) Para $\gamma_{1}, \gamma_{2} \in \Gamma$ com $\gamma_{1}(x)=\gamma_{2}(x)$ para todo $x \in D\left(\gamma_{1}\right) \cap D\left(\gamma_{2}\right)$ definimos

$$
\left(\gamma_{1} \cup \gamma_{2}\right)(x)= \begin{cases}\gamma_{1}(x) & \text { se } x \in D\left(\gamma_{1}\right) \\ \gamma_{2}(x) & \text { se } x \in D\left(\gamma_{2}\right)\end{cases}
$$

Logo, se $\gamma_{1} \cup \gamma_{2}: D\left(\gamma_{1}\right) \cup D\left(\gamma_{2}\right) \rightarrow R\left(\gamma_{1}\right) \cup D\left(\gamma_{2}\right)$ é um homeomorfismo, então $\gamma_{1} \cup \gamma_{2} \in \Gamma$

v) Se $\gamma \in \Gamma$ e $S \subset D(\gamma)$, então a restrição de $\gamma$ a $S$ é um elemento se $\Gamma$.

Temos então a definição do pseudogrupo de holonomia.

Definição 2.3: Definimos o pseudogrupo de holonomia de $\mathcal{F}$ para a cobertura regular $\mathcal{U}$ como o pseudogrupo $\Gamma_{\mathcal{U}}$ de difeomorfismos locais $C^{\infty}$, gerado pelos difeomorfismos locais $\gamma_{\alpha \beta}$ de $\Sigma$.

Seja $\tilde{\mathcal{U}}$ outra cobertura regular folheada, vamos supor que $\tilde{\mathcal{U}}$ é mais fina que $\mathcal{U}$ (i.e., para cada $j$ existe $k(j)$ tal que $\left.\tilde{U}_{j} \subset U_{k(j)}\right)$ e também $\tilde{U}_{j}$ é uniforme em $U_{k(j)}$ (i.e., cada placa de $U_{k(j)}$ encontra no máximo uma placa de $\left.\tilde{U}_{j}\right)$ ) então obtemos uma natural identificação entre os correspondentes pseudogrupos de holonomia $\Gamma_{\tilde{\mathcal{U}}} \stackrel{\sim}{\rightarrow} \Gamma_{\mathcal{U}}$. Assim temos o resultado cuja prova pode ser consultada em $[10, p .76]$.

Lema 2.4: Todos os pseudogrupos de holonomia $\Gamma_{\mathcal{U}}$, onde $\mathcal{U}$ é uma cobertura regular de $M$ para a folheação $\mathcal{F}$, são equivalentes de maneira natural.

Agora podemos definir uma medida transversa invariante como segue.

Definição 2.5: Uma medida transversa invariante por holonomia (para $\mathcal{F}$ ), ou simplesmente uma medida transversa invariante (para $\mathcal{F}$ ), é uma medida $\mu$ definida no espaco transverso $\Sigma$ tal que:

i) É finita em compactos.

ii) É invariante sobre o pseudogrupo de holonomia; i.e.,

$$
\mu(\gamma(B))=\mu(B)
$$

para todo $\gamma \in \Gamma_{\mathcal{U}}$ e mensurável $B \subset D(\gamma)$.

Se existe uma medida invariante para $\mathcal{F}$, dizemos que $\mathcal{F}$ admite uma medida transversa invariante. Definimos o suporte de uma medida transversa invariante, como o conjunto de pontos de $x \in M$ tais que, dado $\Sigma^{\prime}$ um disco transversal a $\mathcal{F}$ que contem a $x$, tem-se que $\mu\left(\Sigma^{\prime}\right)>0$. Denotamos ao suporte de $\mu$ por $\operatorname{supp}(\mu)$. 
Exemplo 2.6: Se $L$ é uma folha fechada de $\mathcal{F}$ e $N$ é uma $q$-variedade transversal a $\mathcal{F}$, então podemos definir uma medida $\mu \mathrm{em} N$ como segue. Para qualquer conjunto $A \subset N$ mensurável seja $\mu(A)$ o número de elementos de $L \cap A$ e que é finito pelo Teorema 1.5 quando $A$ é compacto, e claramente por Teorema $1.4 \mu$ é $\mathcal{F}$-invariante.

Exemplo 2.7: Seja $\omega$ uma 1-forma fechada não singular de classe $C^{r}$. Sabemos que $\omega$ induz uma folheação $\mathcal{F}$ de codimensão 1 de classe $C^{r}$ (ver $[6, p .82]$ ), então para todo disco transversal $\Sigma$ de $\mathcal{F}$, a restrição de $\left.\omega\right|_{\Sigma}$ é uma forma de volumem que é positiva em abertos; por tanto, $\omega$ induz uma medida no espaço transverso de $\mathcal{F}$ que é finita em compactos e como $\omega$ é fechado segue-se que dita forma é invariante pelo pseudogrupo de holonomia de $\mathcal{F}$.

Seja $\mathcal{F}$ uma folheação $p$-dimensional orientável de $M$ que vamos supor que é compacta. Seja $\mathcal{U}=\left\{U_{\alpha}\right\}_{\alpha \in I}$ um atlas regular folheado, $\Sigma=\bigsqcup \Sigma_{\alpha}$ o espaço transverso, $\gamma_{\alpha \beta}: D\left(\lambda_{\alpha \beta}\right) \Sigma_{\alpha} \rightarrow R\left(\lambda_{\alpha \beta}\right) \Sigma_{\beta}$ os difeomorfismos, $\pi_{\alpha}: U_{\alpha} \rightarrow \Sigma_{\alpha}$ a projeção canônica para cada $\alpha \in I$ e $\Gamma=\Gamma_{\mathcal{U}}$ o pseudogrupo de holonomia gerado por $\Gamma_{1}=\left\{\gamma_{\alpha \beta} ; \alpha, \beta \in I\right\}$.

Seja $\mu$ uma medida em $\Sigma$ transversa invariante por holonomia. Então, para qualquer $U_{\alpha} \in \mathcal{U}$, podemos integrar uma $p$-forma $\omega$ sobre cada placa $\pi_{\alpha}^{-1}(x)$. Esto define uma função continua de $\Sigma_{\alpha}$ a $\mathbb{R}$, que ao ser integrada com respeito à medida $\left.\mu\right|_{\Sigma_{\alpha}}$ se obtêm uma aplicação linear real $C_{\mu}$ do conjunto das $p$-formas, como segue:

Se $\left\{\lambda_{\alpha}\right\}_{\alpha \in I}$ é uma partição da unidade associada a $\mathcal{U}$, definimos a aplicação linear

$$
<\Phi_{\mu}, \omega>:=\sum_{\alpha \in I} \int_{\Sigma_{\alpha}}\left(\int_{\pi_{\alpha}^{-1}(x)} \lambda_{\alpha} \omega\right) d \mu(x)
$$

Notemos que esta definição não depende da partição da unidade escolhida, já que se $\operatorname{supp}(\omega) \subset U_{\beta}$ para algum $\beta \in I$, como os difeomorfismos locais $\gamma_{\alpha \beta} \in \Gamma$, são invariantes sobre $\mu$, então

$$
\begin{aligned}
<\Phi_{\mu}, \omega> & =\sum_{\alpha \in I} \int_{\Sigma_{\alpha}}\left(\int_{\pi_{\alpha}^{-1}(x)} \lambda_{\alpha} \omega\right) d \mu(x) \\
& =\sum_{\alpha \in I} \int_{\Sigma_{\beta}}\left(\int_{\pi_{\alpha}^{-1}\left(\gamma_{\beta \alpha}(y)\right)} \lambda_{\alpha} \omega\right) d \mu(y) \\
& =\sum_{\alpha \in I, U_{\alpha} \cap U_{\beta} \neq \emptyset} \int_{\Sigma_{\beta}}\left(\int_{\pi_{\beta}^{-1}(y)} \lambda_{\alpha} \omega\right) d \mu(y) \\
& =\int_{\Sigma_{\beta}}\left(\int_{\pi_{\beta}^{-1}(y)} \omega\right) d \mu(y)
\end{aligned}
$$


Assim, se $\left\{\delta_{\alpha}\right\}$ é outra partição da unidade associada $\mathcal{U}$, como $\operatorname{supp}\left(\delta_{\beta} \omega\right) \subset U_{\beta}, \operatorname{logo}$ pelo anterior temos que

$$
<\Phi_{\mu}, \delta_{\beta} \omega>=\int_{\Sigma_{\beta}}\left(\int_{\pi_{\beta}^{-1}(x)} \delta_{\beta} \omega\right) d \mu(x)
$$

e segue-se que

$$
<\Phi_{\mu}, \omega>=\sum_{\beta \in I}<\Phi_{\mu}, \delta_{\beta} \omega>=\sum_{\beta \in I} \int_{\Sigma_{\beta}}\left(\int_{\pi_{\beta}^{-1}(x)} \delta_{\beta} \omega\right) d \mu(x)
$$

De forma análoga se mostra que a corrente é independente da escolha da cobertura. Por outro lado se $\omega$ é uma $p$-forma exata temos que $\left\langle\Phi_{\mu}, \omega\right\rangle=0$, pois se $\omega=d \eta$ e $\left\{\lambda_{\alpha}\right\}_{\alpha \in I}$ é uma partição da unidade associada a $\mathcal{U}$ então $\eta=\sum_{\alpha} \lambda_{\alpha} \eta \Rightarrow d \eta=\sum_{\alpha} d\left(\lambda_{\alpha} \eta\right)$. Logo desde que $\operatorname{supp}\left(d\left(\lambda_{\alpha} \omega\right)\right) \subset U_{\alpha}$ segue de (2.2) que

$$
<\Phi_{\mu}, \omega>=<\Phi_{\mu}, d \eta>=<\Phi_{\mu}, \sum_{\alpha} d\left(\lambda_{\alpha} \eta\right)>=\sum_{\alpha \in I} \int_{\Sigma_{\alpha}}(\underbrace{\int_{\pi_{\alpha}^{-1}(x)} d\left(\lambda_{\alpha} \eta\right)}_{(*)}) d \mu(x)
$$

e usando o Teorema de Stokes na integral $(*)$ temos que $\int_{\pi_{\alpha}^{-1}(x)} d\left(\lambda_{\alpha} \eta\right)=0 \Rightarrow<\Phi_{\mu}, \omega>=$ 0 . Em outras palavras temos bem definido uma aplicação linear

$$
\begin{aligned}
\Phi_{\mu}: H_{c}^{p}(M) & \longrightarrow \mathbb{R} \\
\omega & \mapsto \sum_{\alpha \in I} \int_{\Sigma_{\alpha}}\left(\int_{\pi_{\alpha}^{-1}(x)} \lambda_{\alpha} \omega\right) d \mu(x)
\end{aligned}
$$

Assim pelo Lema de Poincaré $\mu$ induz um elemento no $(n-p)$-ésimo grupo de cohomologia DeRham, pois

$$
\Phi_{\mu} \in\left(H_{c}^{p}(M)\right)^{*} \simeq H^{n-p}(M)
$$

O seguente Teorema é o primeiro resultado principal de este capítulo, ele é um bonito teorema por que em seu enunciado e sua prova faz uso de varias áreas da matemática. Pode ser pensado como um Teorema abstrato de Poincaré-Bendixson para folheações.

Teorema 2.8: Seja $M^{n}$ uma variedade diferenciável de classe $C^{\infty}$ e $\mathcal{F}$ uma folheação transversalmente orientável de classe $C^{r}(r \geq 1)$ e codimensão $k$ em $M$. Se $N$ é uma subvariedade compacta de dimensão $k$ sem bordo, transversal a $\mathcal{F}$ e que intersecta o suporte de uma medida transversa invariante por holonomia $\mu$, então $N$ representa um elemento não nulo de $H^{n-k}(M)$.

Demonstração. Vamos construir uma $(n-k)$-forma fechada representado por $N$. Seja $T$ uma vizinhança tubular de $N$ em $M$ então ele define um fibrado onde as fibras são as folhas que estão em $T$ (ver figura(2.1)). 


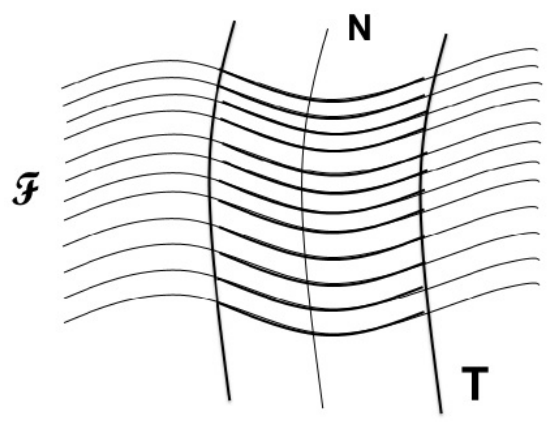

Figura 2.1: $T$ vizinhança tubular da subvariedade $N$.

Como $N$ é compacto, pela Teorema 1.14 a vizinhança tubular $T$ é equivalente a fibrado normal, i.e., as fibras do fibrado normal são levados difeomorficamente nas fibras da vizinhança tubular. Então podemos olhar a vizinhança tubular como o fibrado normal. Seja $\left\{V_{i}\right\}_{i \in I}$ uma cobertura de $N$ tal que trivializa o fibrado, esto é, para cada $i$ existe $\phi_{i}$ difeomorfismo que faz comutar o seguente diagrama

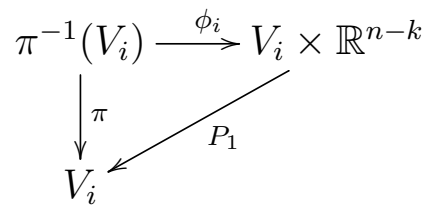

onde $\pi$ e $P_{1}$ são como na definição de espaço fibrado. Seja $f: \mathbb{R}^{n-k} \rightarrow \mathbb{R}$ definido por $f(x)=g\left(\|x\|^{2}\right)$ onde

$$
g(t)= \begin{cases}\exp \left(-\frac{1}{1-t^{2}}\right) & \text { se }|t|<1 \\ 0 & \text { se }|t| \geq 1\end{cases}
$$

então $f$ tem as seguentes propriedades:

i) Tem suporte compacto.

ii) É invariante sob a ação das matrizes ortogonais $\mathcal{O}(n-k)$; i.e., $f(A x)=f(x)$ se $A \in \mathcal{O}(n-k)$

iii) $\int_{\mathbb{R}^{n-k}} f=1$

Assim temos em $V_{i} \times \mathbb{R}^{n-k}$ a seguinte $(n-k)$-forma fechada

$$
\omega=f\left(x_{k+1}, \cdots, x_{n}\right) d x_{k+1} \wedge \cdots \wedge d x_{n}
$$

$\operatorname{Logo} \eta_{i}=\phi_{i}^{*}(\omega)$ é uma $(n-k)$-forma fechada em $\pi^{-1}\left(V_{i}\right)$ para cada $i \in I$.

Afirmação 1: $\eta_{i}=\eta_{j}$ em $\pi^{-1}\left(V_{i}\right) \cap \pi^{-1}\left(V_{j}\right)$. De fato, sabemos que o fibrado normal é 
um fibrado vetorial com grupo estrutural $G L\left(\mathcal{R}^{n-k}\right)$, mas pela Proposição 1.11 podemos reduzir este grupo estrutural a $\mathcal{O}(n-k)$. Logo pela definição 1.7 existe um cociclo $\delta_{i j}: V_{i} \cap V_{j} \rightarrow \mathcal{O}(n-k)$ tal que

$$
\begin{array}{cc}
\phi_{i} \circ \phi_{j}^{-1}:\left(V_{i} \cap V_{j}\right) \times \mathbb{R}^{n-k} & \longrightarrow \\
(z, w) & \mapsto\left(V_{i} \cap V_{j}\right) \times \mathbb{R}^{n-k} \\
& \left(z,\left(\rho \circ \delta_{i j} z\right) w\right)
\end{array}
$$

onde

$$
\begin{array}{cl}
\rho: \mathcal{O}(n-k) & \longrightarrow \text { Diff }{ }^{\infty}\left(\mathbb{R}^{n-k}\right) \\
A & \mapsto \rho(A) w=A w
\end{array}
$$

é a ação de $\mathcal{O}(n-k)$ em $\mathcal{R}^{n-k}$. Logo

$$
\begin{aligned}
\left(\phi_{i} \circ \phi_{j}^{-1}\right)^{*} & (\overbrace{f\left(x_{k+1}, \cdots, x_{n}\right)}^{f_{1}\left(z, x_{k+1}, \cdots, x_{n}\right)} d x_{k+1} \wedge \cdots \wedge d x_{n})= \\
& =f_{1} \circ\left(\phi_{i} \circ \phi_{j}^{-1}\right)(z, w)\left(\phi_{i} \circ \phi_{j}^{-1}\right)^{*}\left(d x_{k+1}\right) \wedge \cdots \wedge\left(\phi_{i} \circ \phi_{j}^{-1}\right)^{*}\left(d x_{n}\right) \\
& =f_{1}\left(z,\left(\rho \circ \delta_{i j} z\right) w\right) d\left(\left(\phi_{i} \circ \phi_{j}^{-1}\right)^{*} x_{k+1}\right) \wedge \cdots \wedge d\left(\left(\phi_{i} \circ \phi_{j}^{-1}\right)^{*} x_{n}\right) \\
& =f\left((\underbrace{\rho \circ \delta_{i j} z}_{\in \mathcal{O}(m-k)}) w\right) d\left(x_{k+1} \circ \phi_{i} \circ \phi_{j}^{-1}(z, w)\right) \wedge \cdots \wedge d\left(x_{n} \circ \phi_{i} \circ \phi_{j}^{-1}(z, w)\right)
\end{aligned}
$$

como $f$ é invariante sob a ação das matrizes ortogonais $\mathcal{O}(n-k)$ segue do anterior que

$$
\left(\phi_{i} \circ \phi_{j}^{-1}\right)^{*}(\omega)=f\left(w_{k+1}, \cdots, w_{n}\right) d w_{k+1} \wedge \cdots \wedge d w_{n}
$$

Por tanto $\phi_{i}^{*} \omega=\phi_{j}^{*} \omega$ o qual conclui a afirmação. Assim podemos definir a $(n-k)$-forma fechada em $T$ como

$$
\eta=\bigsqcup_{i} \eta_{i}
$$

o qual esta bem definida pela afirmação anterior, tem suporte compacto pois $f$ tinha suporte compacto e pode ser estendido a $M$ como

$$
\eta= \begin{cases}\eta & \text { em } T \\ 0 & \text { em outro caso }\end{cases}
$$

Afirmação 2: $\left\langle\Phi_{\mu}, \eta\right\rangle=\mu(N)$. De fato, seja $\mathcal{U}$ uma cobertura regular folheada e $U_{\beta} \in \mathcal{U}$ tal que $U_{\beta} \cap T \neq \emptyset$ que podemos supor que é como na figura 2.2. 


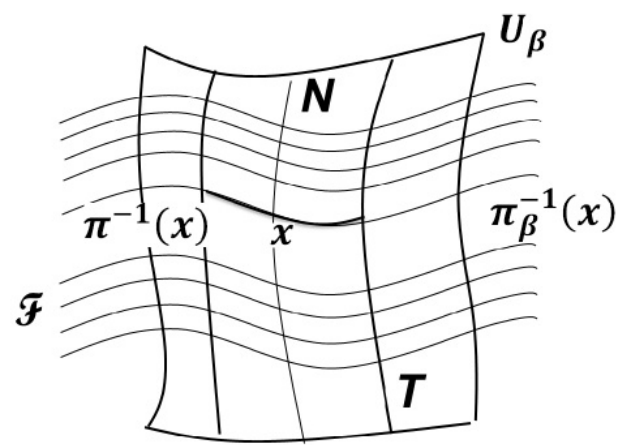

Figura 2.2:

então

$$
\int_{\pi_{\beta}^{-1}(x)} \eta=1
$$

pois

$$
\int_{\pi_{\beta}^{-1}(x)} \eta=\int_{\pi^{-1}(x)} \eta=\int_{\pi^{-1}(x)} \phi_{i}^{*}\left(f d x_{k+1} \wedge \cdots \wedge d x_{n}\right) \text { para algum } i \in I
$$

como $\pi^{-1}(x) \simeq\{x\} \times \mathcal{R}^{n-k}$ por $\phi_{i}$ então segue da ultima linha

$$
\begin{aligned}
\int_{\pi_{\beta}^{-1}(x)}^{\eta} & =\int_{\{x\} \times \mathcal{R}^{n-k}}\left(\phi^{-1}\right)^{*} \circ \phi_{i}^{*}\left(f d x_{k+1} \wedge \cdots \wedge d x_{n}\right) \\
& =\int_{\{x\} \times \mathcal{R}^{n-k}} f d x_{k+1} \wedge \cdots \wedge d x_{n} \\
& =\int_{\mathcal{R}^{n-k}} f=1
\end{aligned}
$$

Por outro lado de (2.1)

$$
\begin{aligned}
\left\langle\Phi_{\mu}, \eta\right\rangle & =\sum_{\beta} \int_{\Sigma_{\beta}}\left(\int_{\pi_{\beta}^{-1}(x)} \lambda_{\beta} \eta\right) d \mu(x) \\
& =\sum_{U_{\beta} \cap N \neq \emptyset} \int_{\Sigma_{\beta}}\left(\int_{\pi_{\beta}^{-1}(x)} \lambda_{\beta} \eta\right) d \mu(x)
\end{aligned}
$$

Como já foi provado que o lado direito da igualdade anterior não depende da escolha da partição da unidade, então podemos tomar uma partição da unidade tal que $\operatorname{supp} \lambda_{\alpha} \cap$ $\operatorname{supp} \lambda_{\beta}=\emptyset$ se $\alpha \neq \beta$; i.e., $\left.\lambda_{\beta}\right|_{\operatorname{supp} \lambda_{\beta}}=1$, então

$$
\left\langle\Phi_{\mu}, \eta\right\rangle=\sum_{\beta} \int_{U_{\beta} \cap N}(\underbrace{\int_{\pi_{\beta}^{-1}(x)} \eta}_{1 \text { por }(2.3)}) d \mu(x)=\sum_{\beta} \int_{U_{\beta} \cap N} d \mu(x)
$$


Ainda mais, fazendo uma refinação dos abertos $U_{\beta}$ tal que $U_{\alpha} \cap U_{\beta}$ seja como máximo uma placa, então de (2.4) segue-se a afirmação.

Como $N$ intersecta o suporte de $\mu$, então por definição de suporte $\mu(N)>0$ e pela afirmação 2 temos que $\left\langle\Phi_{\mu}, \eta\right\rangle>0$. Logo $\eta$ não é uma forma exata. Assim $\eta$ é um elemento não nulo de $H^{n-k}(M)$.

Uma pergunta natural que surge é a seguente: Dada uma folheação $\mathcal{F}$ ele sempre admite uma medida transversa invariante pela holonomia?. A resposta é que geral não é certo. Um exemplo de uma folheação que não admite medida invariante pode ser encontrado na tésis doutoral de [9], este exemplo não é exibido neste trabalho devido, que para seu entendimento precisamos de pré-requisitos que escapam do objetivo deste trabalho. Assim agora nos podemos perguntar. Quais são as condições suficientes e necessárias para a existência de uma medida transversa invariante?. Nas seguintes seções desenvolveremos tópicos para dar as condições suficientes.

\subsection{Crescimento de funções}

Definição 2.9: $\operatorname{Sejam~} G, H: \mathbb{Z}^{+} \rightarrow \mathbb{R}^{+}$duas funções não decrescentes.

a) Se diz que $G$ domina a $H$ o qual denotamos por $G \succeq H$ se existem inteiros positivos $\alpha, \beta$ e $\gamma$ tais que

$$
\alpha G(\beta r) \geq H(r), \forall r \geq \gamma
$$

b) Se $G \succeq H \succeq G$, dizemos que $G$ e $H$ tem o mesmo tipo de crescimento.

c) Se $G \succeq H$ e $H \nsucceq G$, dizemos que $G$ domina estritamente a $H$ e escrevemos $G \succ H$.

\section{Observação 2.10:}

1. A relação $G \succeq H \succeq G$ é de equivalência no conjunto das funções $G: \mathbb{Z}^{+} \rightarrow \mathbb{R}^{+}$.

2. A classe de equivalência de $G$ será denotado por $\operatorname{gr}(G)$ que é chamado tipo de crescimento de $G$.

Exemplo 2.11: As funções exponenciais $r \mapsto a^{r}, a>1$ tem todas o mesmo tipo de crescimento, pois se $a, b>1$, seja $\beta>\log _{a} b$ então

$$
a^{\beta r}>b^{r}, \forall r \geq 1
$$


i.e. $a^{r} \succeq b^{r}$, a relação inversa é análogo.

Exemplo 2.12: Seja $P(r), Q(r)$ polinômios de grau $p$ e $q$ respectivamente, com coeficientes positivos, se $p>q \Rightarrow P \succ Q$, pois $\lim _{r \rightarrow+\infty} \frac{P(r)}{Q(r)}=+\infty$ e segue que $\exists C>0$ tal que $P(r) \geq Q(r), \forall r>C$. Por outro lado suponha que $Q \succeq P \Rightarrow \exists \alpha, \beta$ e $\gamma$ tal que $\alpha Q(\beta r) \geq P(r) \forall r>\gamma \Rightarrow \lim _{r \rightarrow+\infty} \frac{P(r)}{\alpha Q(\beta r)} \leq 1$, logo $p \leq q$ o qual é uma contradição. Assim segue-se que

$$
P \succeq Q \succeq P \Longleftrightarrow p=q
$$

Definição 2.13: Seja $f: \mathbb{Z}^{+} \rightarrow \mathbb{R}^{+}$, dizemos que $f$ tem crescimento exponencial se $\operatorname{gr}(f)=\operatorname{gr}\left(e^{r}\right)$ e se $f \prec e^{r}$ dizemos que $f$ tem crescimento sub-exponencial ou não exponencial.

Definição 2.14: Seja $f: \mathbb{Z}^{+} \rightarrow \mathbb{R}^{+}$não decrescente, dizemos que $f$ tem crescimento polinomial de grau $p$, se existe $P(r)$ polinômio de grau $p$ com coeficientes positivos tal que $\operatorname{gr}(f)=\operatorname{gr}(P)$.

Proposição 2.15: Seja $f: \mathbb{Z}^{+} \rightarrow \mathbb{R}^{+}$não decrescente. $f \succeq e^{r}$ se, e somente se,

$$
\liminf _{r \rightarrow+\infty} \frac{\log f(r)}{r}>0
$$

Demonstração. Como $\liminf _{r \rightarrow+\infty} \frac{\log f(r)}{r}>0 \Rightarrow \exists a, C>0$ tal que

$$
\frac{\log f(r)}{r}>a, \forall r \geq C \Rightarrow \log f(r)>a r, \forall r \geq C
$$

se $b=e^{a}>1 \Rightarrow f(r) \geq e^{a r}=b^{r}, \forall r \geq C$. Logo $f \succeq b^{r}$. Reciprocamente, se existem $\alpha, \beta$ e $\gamma \in \mathbb{Z}^{+}$tal que

$$
f(\beta r) \geq \frac{e^{r}}{\alpha}, \forall r \geq \gamma
$$

Considerando inteiros $k$ no intervalo $\beta r \leq k \leq \beta(r+1)$, concluirmos que

$$
f(k) \geq f(\beta r) \geq \frac{e^{r}}{\alpha} \geq \frac{e^{k-\beta}}{\alpha}, \forall k \geq \beta \gamma
$$

aplicando log temos que

$$
\frac{\log f(k)}{k} \geq \frac{1}{\beta}-\frac{1+\log \alpha}{k}, \forall k \geq \beta \gamma
$$

Assim

$$
\lim _{k \rightarrow \infty} \inf \frac{\log f(k)}{k} \geq \frac{1}{\beta}>0 .
$$


Corolário 2.16: $\liminf _{r \rightarrow+\infty} \frac{\log f(r)}{r}=0 \Longleftrightarrow f$ tem crescimento sub-exponencial.

Exemplo 2.17: A função $r \mapsto r^{r^{s}}$, não tem crescimento polinomial nem exponencial. Além disso se $s \neq s^{\prime}$, então $g r\left(r^{r^{s}}\right) \neq \operatorname{gr}\left(r^{r^{s^{\prime}}}\right)$.

\subsection{Condições suficientes para a existência de uma me- dida invariante}

Seja $X$ um espaço topológico e $\Gamma$ um pseudogrupo de homeomorfismos locais de $X$. Dizemos que $\Gamma$ é gerado por um subconjunto $\Gamma_{1} \subseteq \Gamma$, se cada elemento de $\Gamma$ pode ser obtido de elementos de $\Gamma_{1}$ por um número finito de operações dados na definição 2.2. $\Gamma_{1}$ é chamado um conjunto gerador para $\Gamma$. Dizemos que $\Gamma$ é finitamente gerado e enumeravelmente gerado se $\Gamma_{1}$ é finito e enumerável. Dizemos que o conjunto gerador $\Gamma_{1}$ é simétrico se $\gamma \in \Gamma_{1}$ temos que $\gamma^{-1} \in \Gamma_{1}$. De aqui em diante vamos assumir que todo conjunto gerador é simétrico.

Definição 2.18: Dado $x \in X$ definimos a órbita de $x$ sobre $\Gamma$ como o conjunto

$$
\Gamma(x)=\{y \in X: y=\gamma(x), \text { para algúm } \gamma \in \Gamma\}
$$

Observação 2.19: Se $y \in \Gamma(x)$, então $\Gamma(x)=\Gamma(y)$.

Seja $\Gamma$ é finitamente gerado e $\Gamma_{1}$ um conjunto gerador finito e simétrico, definimos

$$
\Gamma^{n}(x)=\left\{y \in X: y=\gamma_{j_{1}} \cdots \gamma_{j_{r}}(x), r \leq n \text { e } \gamma_{j_{i}} \in \Gamma_{1}\right\} \subset \Gamma(x)
$$

Assim podemos definir a seguinte função

$$
G_{x}: \mathbb{Z}^{+} \rightarrow \mathbb{R}^{+}
$$

dado por $G_{x}=\left|\Gamma^{n}(x)\right|$, onde $|$.$| denota a cardinalidade de um conjunto. Esta função é$ chamada função de crescimento de $\Gamma$ em $x$ associado a $\Gamma_{1}$ ou função de crescimento da orbita de $x$ sobre $\Gamma$ associado a $\Gamma_{1}$.

O seguinte lema mostra que o tipo de crescimento da função de crescimento de $\Gamma$ não depende do ponto.

Lema 2.20: Se $y \in \Gamma(x)$, então $\operatorname{gr}\left(G_{x}\right)=g r\left(G_{y}\right)$.

Demonstração. Como $y \in \Gamma(x) \Rightarrow y=\gamma(x)$, para algum $\gamma \in \Gamma$. Como $\Gamma_{1}$ gera $\Gamma$ 


$$
\Rightarrow \gamma=\gamma_{j_{1}} \cdots \gamma_{j_{s}}, \operatorname{com} \gamma_{j_{i}} \in \Gamma_{1}
$$

Se $z \in \Gamma^{k}(y)$, por $(2.5)$ temos que $z=\gamma_{i_{1}} \cdots \gamma_{i_{r}}(y)$. Logo,

$$
z=\gamma_{i_{1}} \cdots \gamma_{i_{r}} \gamma_{j_{1}} \cdots \gamma_{j_{s}}(x) \Rightarrow z \in \Gamma^{k+s}
$$

Assim

$$
\Gamma^{k}(y) \subset \Gamma^{k+s}(x), \forall k \in \mathbb{Z}^{+}
$$

de onde segue,

$$
G_{y}(k) \leq G_{x}(k+s) \leq G_{x}(2 k), \forall k \geq s
$$

Por tanto $G_{y} \preceq G_{x}$. De forma análoga temos que $G_{x} \preceq G_{y}$. O que conclui a prova.

Nosso seguinte objetivo é mostrar que a função de crescimento de $\Gamma$ em $x$, é independente do conjunto gerador.

Lema 2.21: Se $\Gamma_{1}^{\prime}=\left\{\alpha_{i}\right\}_{i=1}^{n}$ é outro conjunto gerador de $\Gamma$ finito e simétrico. Se $\Gamma_{1} \subset \Gamma_{1}^{\prime}$, então $G_{x}(k) \leq G_{x}^{\prime}(k), \forall k \in \mathbb{Z}^{+}$onde $G_{x}, G_{x}^{\prime}$ são as funções de crescimento associadas a $\Gamma_{1}, \Gamma_{1}^{\prime}$ respectivamente.

Demonstração. Desde que $\Gamma_{1} \subset \Gamma_{1}^{\prime}$ e por (2.5), temos que $\Gamma^{k}(x) \subset\left(\Gamma^{\prime}\right)^{k}(x), \forall k \in \mathbb{Z}^{+}$. $\operatorname{Logo} G_{x}(k) \leq G_{x}^{\prime}(k), \forall k \in \mathbb{Z}^{+}$.

\section{Observação 2.22:}

1. O conjunto $\Gamma_{N}=\left\{\gamma_{j_{1}} \cdots \gamma_{j_{r}} / r \leq N, \gamma_{j_{i}} \in \Gamma_{1}\right\}$ com $N \geq 1$ é um conjunto gerador de $\Gamma$ finito e simétrico tal que $\Gamma_{1} \subseteq \Gamma_{N}$.

2. Se $G_{x}^{N}$ é a função de crescimento de $\Gamma$ associado a $\Gamma_{N}$, então temos $G_{x}^{N}(k) \leq G_{x}(N k)$.

Agora, sejam $\Gamma_{1}$ e $\Gamma_{1}^{\prime}$ dois conjuntos geradores finitos e simétricos de $\Gamma$, tomando $N$ inteiro positivo tal que $\Gamma_{1}^{\prime} \subset \Gamma_{N}$, segue-se do Lema (2.21) e da observação (2.22) que $G_{x}^{\prime}(k) \leq G_{x}^{N}(k) \leq G_{x}(N k), \forall k \in \mathbb{Z}^{+}$Assim $G_{x}^{\prime} \preceq G_{x}$. Analogamente $G_{x} \preceq G_{x}^{\prime}$, por tanto

$$
\operatorname{gr}\left(G_{x}\right)=\operatorname{gr}\left(G_{x}^{\prime}\right)
$$

Assim temos a seguinte definição, que pelos argumentos anteriores esta bem definido.

Definição 2.23: Se $\Gamma$ é um pseudogrupo de homeomorfismos locais em $X$ e $x \in X$, então o crescimento de $\Gamma$ em $x$ se define como $\operatorname{gr}\left(G_{x}\right)$. 
Observação 2.24: $G_{x}$ sempre é dominado por uma função exponencial, pois se $\Gamma_{1}$ é um conjunto gerador finito de $\Gamma$ com $m$ elementos, segue de (2.5) que

$$
\left|\Gamma^{k}(x)\right| \leq m+m^{2}+\cdots+m^{k} \leq(m+1)^{k}
$$

Se $\Gamma$ é enumeravelmente gerado, dizemos que tem crescimento não exponencial em $x \in X$, se pode-se escrever

$$
\Gamma=\bigcup_{m=1}^{\infty} \Gamma_{m} \operatorname{com} \Gamma_{m} \subset \Gamma_{m+1}
$$

onde $\Gamma_{m}$ são pseudogrupos finitamente gerados cada um das quais tem crescimento não exponencial em $x$

O seguinte resultado é clave para mostrar nosso segundo objetivo deste capítulo. Este Teorema fornece as condições suficientes para a existência de uma medida invariante pelo pseudogrupo de holonomia.

Teorema 2.25: Seja $\Gamma$ um pseudogrupo enumerável gerado de homeomorfismos de um espaço métrico compacto $X$. Se $\Gamma$ tem crescimento sub-exponencial em $x \in X$, então existe uma medida de Borel $\mu$ em $X$ com as seguintes propriedades:

i) $\mu$ é $\Gamma$-invariante.

ii) $\mu(X)=1$

iii) $\operatorname{supp}(\mu) \subseteq \overline{\Gamma(x)}$

Demonstraremos o teorema no primeiro caso quando $\Gamma$ é finitamente gerado. Empezaremos demonstrando dois lemas.

Lema 2.26: Existe uma sequencia de inteiros positivos $\left\{n_{k}\right\}_{k=1}^{\infty}$ com $n_{k} \rightarrow+\infty$ tal que

$$
\lim _{k \rightarrow+\infty} \frac{\left|\Gamma^{n_{k}+1}(x) \backslash \Gamma^{n_{k}-1}(x)\right|}{\left|\Gamma^{n_{k}}(x)\right|}=0
$$

Demonstração. Por absurdo; i.e., $\lim _{k \rightarrow+\infty} \frac{\left|\Gamma^{k+1}(x) \backslash \Gamma^{k-1}(x)\right|}{\left|\Gamma^{k}(x)\right|}>a>0$. Então $\exists N>0$ tal que

$$
\text { se } k \geq N \Rightarrow\left|\Gamma^{k+1}(x) \backslash \Gamma^{k-1}(x)\right|>a\left|\Gamma^{k}(x)\right| \geq a\left|\Gamma^{k-1}(x)\right|
$$

$\log \mathrm{O}$

$$
\left|\Gamma^{k+1}(x) \backslash \Gamma^{k-1}(x)\right|+\left|\Gamma^{k-1}(x)\right| \geq(1+a)\left|\Gamma^{k-1}(x)\right|
$$




$$
\Rightarrow\left|\Gamma^{k+1}(x)\right| \geq(1+a)\left|\Gamma^{k-1}(x)\right|, \forall k \geq N
$$

De (2.6), para todo $s>0$ temos que

$$
\left|\Gamma^{N-2 s-1}(x)\right| \geq(a+1)^{s}\left|\Gamma^{N-1}(x)\right| \text { e }\left|\Gamma^{N-2 s}(x)\right| \geq(a+1)^{s}\left|\Gamma^{N}(x)\right|
$$

assim, tomando $s>N$ temos que

$$
\left|\Gamma^{3 s}(x)\right| \geq(1+a)^{s}\left|\Gamma^{N-1}(x)\right| \geq\left|\Gamma^{N-1}(x)\right| \Rightarrow G_{x}(3 s) \geq(1+a)^{s}, \forall s>N
$$

desta ultima linha, da definição (2.23) da Observação (2.24), segue-se que $\Gamma$ tem crescimento exponencial em $x$ o qual é uma contradição com a hipótese.

Definição 2.27: Dado $\gamma \in \Gamma$ e $f \in C(X) \operatorname{com} \operatorname{supp}(f) \subseteq R(\gamma)$ defina $f_{\gamma} \in C(X)$ por

$$
f_{\gamma}(y)=\left\{\begin{array}{cc}
f(\gamma(y)) & \text { se } y \in D(\gamma) \\
0 & \text { caso contrario }
\end{array}\right.
$$

Observação 2.28: Como o crescimento de $\Gamma$ em $x$ não depende do conjunto gerador, então vamos supor que $\Gamma_{1}$ é um conjunto gerador de $\Gamma$ tal que

$$
\text { se } \gamma \in \Gamma_{1} \backslash I d_{X} \text { então } D(\gamma) \cap R(\gamma)=\emptyset \text {. }
$$

Lema 2.29: Seja $\Gamma_{1}$ um conjunto gerador finito de $\Gamma$. Se $\gamma \in \Gamma_{1}$ e $f \in C(X)$ com $\operatorname{supp}(f) \subset R(\gamma)$ e $n \geq 1$ inteiro, então

$$
\sum_{y \in \Gamma^{n}(x)}\left(f(y)-f_{\gamma}(y)\right)=\sum_{z \in \Gamma^{n}(x) \triangle \gamma \Gamma^{n}(x)} \epsilon_{z} f(z)
$$

onde

$$
\epsilon_{z}(y)=\left\{\begin{array}{cc}
1 & \text { se } z \in \Gamma^{n}(x) \\
-1 & \text { se } z \in \gamma \Gamma^{n}(x)
\end{array}\right.
$$

Demonstração. Se $\gamma=I d_{X}$ é obvio. Então vamos supor que $\gamma \neq I d_{X}$. Por Observação $(2.28)$

$$
\text { se } y \in D(\gamma) \Rightarrow y \notin R(\gamma) \Rightarrow f(y)=0, \operatorname{pois} \operatorname{supp}(f) \subset R(\gamma)
$$

além disso,

$$
\text { se } y \notin D(\gamma) \Rightarrow f_{\gamma}(y)=0
$$


assim,

$$
\begin{aligned}
\sum_{y \in \Gamma^{n}(x)}\left(f(y)-f_{\gamma}(y)\right) & =\sum_{y \in \Gamma^{n}(x) \cap D(\gamma)}\left(f(y)-f_{\gamma}(y)\right)+\sum_{y \in \Gamma^{n}(x) \cap D(\gamma)}\left(f(y)-f_{\gamma}(y)\right) \\
& =\sum_{y \in \Gamma^{n}(x) \backslash D(\gamma)} f(y)+\sum_{y \in \Gamma^{n}(x) \backslash D(\gamma)} f(y)
\end{aligned}
$$

desde que

$$
z \in \gamma \Gamma^{n}(x) \Leftrightarrow \exists ! y \in \Gamma^{n}(x) \cap D(\gamma) \text { tal que } z=\gamma(y)
$$

segue que

$$
\sum_{y \in \Gamma^{n}(x) \cap D(\gamma)}-f_{\gamma}(y)=\sum_{y \in \Gamma^{n}(x) \cap D(\gamma)}-f(\gamma(y))=\sum_{z \in \gamma \Gamma^{n}(x)}-f(z)=\sum_{z \in \gamma \Gamma^{n}(x)} \epsilon_{z} f(z)
$$

e desde que $\operatorname{supp}(f) \subset R(\gamma)$ temos

$$
\sum_{y \in \Gamma^{n}(x) \backslash D(\gamma)} f(y)=\sum_{y \in \Gamma^{n}(x) \backslash D(\gamma)} f(y)+\underbrace{\sum_{y \in D(\gamma)} f(y)}_{0}=\sum_{y \in \Gamma^{n}(x)} f(y)=\sum_{z \in \Gamma^{n}(x)} \epsilon_{z} f(z)
$$

Logo de (2.8), (2.9) e (2.7) segue que

$$
\sum_{y \in \Gamma^{n}(x)}\left(f(y)-f_{\gamma}(y)\right)=\sum_{z \in \Gamma^{n} \triangle \gamma \Gamma^{n}(x)} \epsilon_{z} f(z)
$$

Definição 2.30: Seja $\phi: C(X) \rightarrow \mathbb{R}$ uma funcional linear, dizemos que $\phi$ é $\Gamma$-invariante se para cada $\gamma \in \Gamma_{1}$ e $f \in C(X) \operatorname{com} \operatorname{supp}(f) \subset R(\gamma)$ temos que $\phi\left(f_{\gamma}\right)=\phi(f)$. Dizemos que $\phi$ é normalizada se $\phi(1)=1$.

Demostração do Teorema 2.25. Estamos supondo que $\Gamma$ é finitamente gerado.

Afirmação 1: Existe $I: C(X) \rightarrow \mathbb{R}$ um funcional linear $\Gamma$-invariante e normalizada. De fato, seja $\left\{n_{k}\right\}_{k=1}^{\infty}$ uma sequencia como no lema 2.26 , então para cada $k \geq 1$, definimos a aplicação linear $I_{k}: C(X) \rightarrow \mathbb{R}$ dado por

$$
I_{k}(f)=\frac{1}{\mid \Gamma^{n_{k}}(x)} \sum_{y \in \Gamma^{n_{k}}(x) \mid} f(y)
$$

Assim definidas estas funcionais lineares são normalizados e limitadas. Como $C(X)$ é separável, então pelo por um teorema analise funcional, existe uma subsequencia de $\left\{I_{k}\right\}$ tal que converge na topologia fraca* para uma aplicação linear, sem perda de generalidade vamos tomar a subsequencia como toda a sequencia, então $\exists I: C(X) \rightarrow \mathbb{R}$ aplicação linear tal que $I_{k} \stackrel{*}{\rightarrow} I$ quando $k \rightarrow+\infty$; i.e., 


$$
I_{k} \stackrel{*}{\rightarrow} I \text { quando } k \rightarrow+\infty \Leftrightarrow \lim _{k \rightarrow+\infty} I_{k}(f)=I(f), \forall f \in C(X)
$$

Assim temos que $I$ é normalizado, pois os $I_{k}$ são. Agora mostraremos que $I$ é $\Gamma$ invariante. Seja $\gamma \in \Gamma_{1}$ e $f \in C(X) \operatorname{com} \operatorname{supp}(f) \subset R(\gamma)$, então

$$
\left\{z \in \Gamma^{n_{k}-1}(x): f(z) \neq 0\right\} \subset \gamma \Gamma^{n_{k}}(x)
$$

pois, se $z \in\left\{z \in \Gamma^{n_{k}-1}(x): f(z) \neq 0\right\} \Rightarrow z \in R(\gamma)$, logo $z \in \Gamma^{n_{k}-1}(x) \cap R(\gamma) \Rightarrow z=$ $\gamma \gamma^{-1}(z) \in \gamma \Gamma^{n_{k}}(x)$ e de (2.12) temos que

$$
\Gamma^{n}(x) \triangle \gamma \Gamma^{n}(x) \subset \Gamma^{n+1}(x) \backslash\left\{z \in \Gamma^{n-1}(x) ; f(z) \neq 0\right\}
$$

Por outro lado, de (2.10) e (2.11) temos que

$$
\left|I(f)-I\left(f_{\gamma}\right)\right|=\left|\lim _{k \rightarrow+\infty} \frac{1}{\left|\Gamma^{n_{k}}(x)\right|} \sum_{y \in \Gamma^{n_{k}}(x)}\left(f(y)-f_{\gamma}(y)\right)\right|
$$

e pelo Lema 2.29 segue-se que

$$
\left|I(f)-I\left(f_{\gamma}\right)\right|=\left|\lim _{k \rightarrow+\infty} \frac{1}{\left|\Gamma^{n_{k}}(x)\right|} \sum_{y \in \Gamma^{n_{k}}(x) \triangle \gamma \Gamma^{n_{k}}(x)} \epsilon_{y} f(y)\right|
$$

e usando a expressão (2.13) temos

$$
\begin{aligned}
\left|I(f)-I\left(f_{\gamma}\right)\right| & \leq \lim _{k \rightarrow+\infty} \frac{1}{\left|\Gamma^{n_{k}}(x)\right|} \sum_{y \in \Gamma^{n_{k}+1}(x) \backslash \Gamma^{n_{k}-1}}(x)|f(y)| \\
& \leq \lim _{k \rightarrow+\infty} \frac{1}{\left|\Gamma^{n_{k}}(x)\right|} \sup _{y \in X}|f(y)|\left|\Gamma^{n_{k}+1}(x) \backslash \Gamma^{n_{k}-1}(x)\right|
\end{aligned}
$$

assim, pelo Lema 2.26 segue-se que $I(f)=I\left(f_{\gamma}\right)$. Logo, pelo Teorema de representação de Riesz, temos que existe uma única medida $\mu$ de Borel em $X$ tal que

$$
I(f)=\int_{X} f d \mu, \text { para todo } f \in C(X)
$$

como $I(1)=1$, segue-se $\mu(X)=1$, desde que $I$ é $\Gamma$-invariante temos que $\mu$ é $\Gamma$-invariante. Mostraremos que $\operatorname{supp}(\mu) \subseteq \overline{\Gamma(x)}$, como

$$
\operatorname{supp}(\mu)=\left\{z \in X ; \text { para toda vizinhança } V_{z} \text { de } z, \mu\left(V_{z}\right)>0\right\}
$$

seja $z \in \operatorname{supp}(\mu)$, como $X$ é métrico, as bolas abertas $B_{\frac{1}{r}}(z)$ são vizinhanças de $z$ com $r \in \mathbb{N}$, então $\mu\left(B_{\frac{1}{r}}(z)\right)>0$. Como $\mu\left(B_{\frac{1}{r}}(z)\right)=I\left(\chi_{B_{\frac{1}{r}}(z)}\right)$ e $\lim _{k \rightarrow+\infty} I_{k}\left(\chi_{B_{\frac{1}{r}}(z)}\right)=I\left(\chi_{B_{\frac{1}{r}}(z)}\right)$. Por outro lado temos

$$
\left.I_{k}\left(\chi_{B_{\frac{1}{r}}(z)}\right)=\frac{1}{\left|\Gamma^{n_{k}}(x)\right|} \sum_{y \in \Gamma^{n_{k}}(x)} \chi_{B_{\frac{1}{r}}(z)}\right)(y)
$$


assim, para cada $r$, existe $k \in \mathbb{Z}^{+}$tal que $I_{k}\left(B_{\frac{1}{r}}(z)\right)>0$, logo existe $z_{r} \in B_{\frac{1}{r}}(z) \cap \Gamma^{n_{k}}(x)$ para todo $r \in \mathbb{Z}^{+}$, então $\lim _{r \rightarrow+\infty} z_{r}=z$ e como $z_{r} \in \Gamma(x)$ segue-se $z \in \overline{\Gamma(x)}$. Assim concluise a prova no caso quando $\Gamma$ é finitamente gerado.

Agora mostraremos o caso geral, como $\Gamma$ é enumeravelmente gerado e tem crescimento não exponencial em $x$, então podemos escrever

$$
\Gamma=\cup_{m=1}^{\infty} \Gamma_{m}
$$

onde, os $\Gamma_{m}$ são finitamente gerado como crescimento não exponencial em $x$ e tal que $\Gamma_{m} \subset \Gamma_{m+1}$. Assim pelo caso já provado temos que para cada $m$ existe $I_{m}: C(X) \rightarrow \mathbb{R}$ funcional linear normalizado $\Gamma_{m}$-invariante, desde que $\Gamma_{m} \subset \Gamma_{m+k}$ para todo $k \geq 0$ então $I_{m+k}$ é $\Gamma_{m}$-invariante para todo $k \geq 0$ e pela forma como foram construídos os $I_{m}$ seguese $\left\{I_{m+k}\right\}_{k \geq 0}$ é uma sequencia de funcionais lineares limitados, então por um teorema de analise funcional,

$$
\exists I: C(X) \rightarrow \mathbb{R} \text { aplicação linear tal que } I_{m+k} \stackrel{*}{\rightarrow} I \text { quando } k \rightarrow \infty
$$

pelas mesmas contas feitas anteriormente temos que $I$ é $\Gamma_{m}$-invariante e normalizado, como $m$ é arbitrário, então $I$ é $\Gamma$-invariante. Pelo Teorema de representação de Riesz, existe uma única medida $\mu \mathrm{em} X$, que fazendo as mesmas contas do caso anterior temos que $\mu(X)=1$ e $\operatorname{supp}(\mu) \subset \overline{\Gamma(x)}$. Assim conclui-se a prova.

O seguinte resultado será enunciado como um lema, por que usaremos este resultado na seguinte seção, na prova do segundo resultado principal deste capítulo.

Lema 2.31: Seja $\Gamma$ um pseudogrupo de homeomorfismos de um espaço de Hausdorff $X$, seja $K \subset X$ um compacto e $\Gamma_{K} \subset \Gamma$ a restrição de $\Gamma$ para $K$. Assumindo que $\Gamma_{K}$ preserva uma (finitamente aditiva) medida finita $\mu_{K}$ definida no anel gerado pelos conjuntos compactos. Suponha que $X$ é coberto por conjuntos abertos da forma $D(\gamma)$ onde $\gamma \in \Gamma$ e $R(\gamma) \subset K$. Então $\mu_{K}$ tem uma única extensão para uma medida $\mu$ que é definida no anel gerado pelos conjuntos compactos de $X$ e é finito em conjuntos compactos.

Demonstração. Seja

$$
R^{\prime}=\left\{A \subset X: A \subset \bigcup_{i \in I} K_{i}, I \text { finito e } K_{i} \text { são compactos em } X\right\}
$$

então temos que $R^{\prime}$ é um anel em $X$ que contém a todos os conjuntos compactos de $X$. Logo, se $R$ é o anel gerado por os conjuntos compactos de $X$ então $R \subset R^{\prime}$. Seja $A \in R \Rightarrow A \in R^{\prime} \Rightarrow A \subset \bigcup_{i \in I} K_{i} \Rightarrow \bar{A} \subset \bigcup_{i \in I} \overline{K_{i}}$ como $X$ é Hausdorff e $K_{i}$ compacto, 
segue que $K_{i}$ é fechado, então $\bar{A} \subset \bigcup_{i \in I} K_{i} \Rightarrow \bar{A}$ é compacto em $X$. Como $X$ é coberto por conjuntos abertos da forma $D(\gamma)$ onde $\gamma \in \Gamma$ e $R(\gamma) \subset K$. Assim

$$
A=\biguplus_{i=1}^{m} A_{i} \text { onde } A_{i} \subset D\left(\gamma_{i}\right) \text { e } R\left(\gamma_{i}\right) \subset K
$$

definamos a seguinte medida em $R$,

$$
\begin{array}{rrrr}
\mu: & R \longrightarrow & {[0,+\infty>} & \\
A & \mapsto & \sum_{i=1}^{m} \mu_{K}\left(\gamma_{i}\left(A_{i}\right)\right)
\end{array}
$$

Afirmação 1: $\mu$ é bem definida. De fato,

i) Se $B \subset D(\gamma)$, com $R(\gamma) \subset K$ e $B \subset D\left(\gamma^{\prime}\right)$, com $R\left(\gamma^{\prime}\right) \subset K$, então $\mu_{K}\left(\gamma^{\prime}(B)\right)=$ $\mu_{K}(\gamma(B))$, pois

$$
\gamma(B) \stackrel{\gamma^{-1}}{\longrightarrow} B \stackrel{\gamma}{\rightarrow} \gamma^{\prime}(B)
$$

então $\gamma^{\prime} \circ \gamma^{-1} \in \Gamma_{K}$ e como $\mu_{K}$ é $\Gamma_{K}$-invariante, temos que

$$
\mu_{K}\left(\gamma^{\prime}(B)\right)=\mu_{K}\left(\gamma^{\prime} \circ \gamma^{-1} \circ \gamma^{\prime}(B)\right)=\mu_{K}(\gamma(B))
$$

ii) Se $A=\biguplus_{j=1}^{n} B_{j}$ onde $B_{j} \in D\left(\gamma_{j}^{\prime}\right)$ e $R\left(\gamma_{j}^{\prime}\right) \subset K$, assim $A_{i}=\biguplus_{j=1}^{n}\left(A_{i} \cap B_{j}\right)$. Desde que $\mu_{K}$ finita aditiva e por (i) temos que

$$
\begin{aligned}
\mu_{K}\left(\gamma_{i}\left(A_{i}\right)\right) & =\sum_{j=1}^{n} \mu_{K}\left(\gamma_{i}\left(A_{i} \cap B_{j}\right)\right) \\
& =\sum_{j=1}^{n} \mu_{K}\left(\gamma_{i}^{\prime}\left(A_{i} \cap B_{j}\right)\right)
\end{aligned}
$$

Logo,

$$
\sum_{i=1}^{m} \mu_{K}\left(\gamma_{i}\left(A_{i}\right)\right)=\sum_{i=1}^{m} \sum_{j=1}^{n} \mu_{K}\left(\gamma_{j}^{\prime}\left(A_{i} \cap B_{j}\right)\right)=\sum_{j=1}^{n} \sum_{i=1}^{m} \mu_{K}\left(\gamma_{i}^{\prime}\left(A_{i} \cap B_{j}\right)\right)=\sum_{j=1}^{n} \mu_{K}\left(\gamma_{i}^{\prime}\left(B_{j}\right)\right)
$$

Afirmação 2: $\mu$ é $\Gamma$-invariante. De fato, seja $A \in R$ tal que $A \in D(\gamma) \operatorname{com} \gamma \in \Gamma$. Se $A=\biguplus_{i=1}^{m} A_{i}$ onde $A_{i} \in D\left(\gamma_{i}\right)$ e $R\left(\gamma_{i}\right) \subset K$, então $\gamma(A)=\biguplus_{i=1}^{m} \gamma\left(A_{i}\right) \Rightarrow \mu(\gamma(A))=$ $\sum_{i=1}^{m} \mu\left(\gamma\left(A_{i}\right)\right) \cdot \mu\left(\gamma\left(A_{i}\right)\right)=\mu\left(A_{i}\right)$, pois, vamos supor por simplicidade que $\gamma\left(A_{i}\right) \subset D\left(\alpha^{\prime}\right)$ e $R\left(\gamma^{\prime}\right)$, para algum $\gamma^{\prime}$, então por definição de $\mu$ temos que

$$
\begin{gathered}
\mu\left(\gamma\left(A_{i}\right)\right)=\mu_{K}\left(\gamma^{\prime}\left(\gamma A_{i}\right)\right) \quad \text { e } \quad \mu\left(A_{i}\right)=\mu_{K}\left(\gamma_{i}\left(A_{i}\right)\right) \\
\gamma^{\prime}\left(\gamma\left(A_{i}\right)\right) \stackrel{\left(\gamma^{\prime}\right)^{-1}}{\longrightarrow} \gamma\left(A_{i}\right) \stackrel{\gamma^{-1}}{\longrightarrow} A_{i} \\
\gamma_{i}\left(A_{i}\right)
\end{gathered}
$$


como $\gamma^{\prime}\left(\gamma\left(A_{i}\right)\right), \gamma_{i}\left(A_{i}\right) \subset K$, então $\gamma_{i} \circ \gamma^{-1} \circ\left(\gamma^{\prime}\right)^{-1} \in \Gamma_{K}$. Logo,

$$
\left.\mu_{K}(\underbrace{\left.\gamma_{i} \circ \gamma^{-1} \circ\left(\gamma^{\prime}\right)^{-1}\right)\left(\gamma^{\prime}(\gamma\right.}_{\gamma_{i}}\left(A_{i}\right))\right)=\mu_{K}\left(\gamma^{\prime}\left(\gamma\left(A_{i}\right)\right)\right) \Rightarrow \mu_{K}\left(\gamma_{i}\left(A_{i}\right)\right)=\mu_{K}\left(\gamma^{\prime}\left(\gamma A_{i}\right)\right)
$$

e de (2.14) segue que $\mu\left(A_{i}\right)=\mu\left(\gamma\left(A_{i}\right)\right)$. O qual prova que $\mu(\gamma(A))=\mu(A)$.

Afirmação 3: $\mu$ é única. De fato, suponha que exista outra medida $\tilde{\mu}$ que é extensão de $\mu_{K}$. Seja $A \in R$ e $A=\biguplus_{i=1}^{m} A_{i}$ onde $A_{i} \in D\left(\gamma_{i}\right)$ e $R\left(\gamma_{i}\right) \subset K$. Como $\tilde{\mu}$ é $\Gamma$-invariante, então

$$
\begin{aligned}
\mu(A) & =\sum_{i=1}^{m} \mu_{K}\left(\gamma_{i}\left(A_{i}\right)\right) \\
& =\sum_{i=1}^{m} \tilde{\mu}\left(\gamma_{i}\left(A_{i}\right)\right) \\
& =\sum_{i=1}^{m} \tilde{\mu}\left(A_{i}\right) \\
& =\tilde{\mu}(A)
\end{aligned}
$$

\subsection{Crescimento em folheações}

Seja $M$ uma variedade compacta e $\mathcal{F}$ uma folheação de codimensão $k$ e de classe $C^{r}$ ( $r \geq 0$ se $k=1, r \geq 1$ em outro caso) e $\mathcal{U}=\left\{U_{\alpha}\right\}_{\alpha=1}^{q}$ uma cobertura regular folheada e $\Sigma=\bigsqcup_{\alpha} \Sigma_{\alpha}$ o espaço transverso, $\gamma_{\alpha \beta}: \Sigma_{\alpha} \rightarrow \Sigma_{\beta}$ os difeomorfismos locais e $\Gamma$ o pseudogrupo de holonomia finitamente gerado por $\Gamma_{1}=\left\{\gamma_{\alpha \beta}: 1 \leq \alpha, \beta \leq q\right\}$ simétrico.

Definição 2.32: Seja $P_{0} \in \Sigma$ uma placa e $L \in \mathcal{F}$ tal que $P_{0} \in L$, dizemos que $L$ tem crescimento exponencial ou sub-exponencial com respeito a $\mathcal{U}$ se $\Gamma$ tem crescimento exponencial ou sub-exponencial em $P_{0}$.

Observação 2.33: Em outras palavras, a função de crescimento de uma folha com respeito $\mathcal{U}$ é a função que atribui a cada inteiro positivo $n$ o número de distintas placas que podem ser alcançadas a partir de uma placa inicial por cadeias de comprimento $\leq n$.

Na seção anterior mostramos que o tipo de crescimento de $\Gamma$ em um ponto $x$ não depende do ponto que se toma em $\Gamma(x)$, assim o tipo de crescimento de uma folha não depende da placa que se tome em $L$. O seguente resultado mostra ainda que o tipo de crescimento da folha é independente da cobertura regular.

Proposição 2.34: O tipo de crescimento de uma folha não depende da cobertura regular. 
Demonstração. Seja $\mathcal{V}=\left\{V_{j}\right\}_{j=1}^{t}$ outra cobertura. Vamos supor que $\mathcal{V}$ é um refinamento de $\mathcal{U}$. Sejam $G$ e $H$ as funções de crescimento de $L$ com respeito a $\mathcal{U}$ e a $\mathcal{V}$ respectivamente. Seja $\left(P_{1}, \cdots, P_{r}\right)$ uma cadeia de placas de $\mathcal{U}$ então cada $P_{i}$ interseca no máximo $t$ placas de $\mathcal{V}$, logo existe uma cadeia de placas $\left(P_{1}^{\prime}, \cdots, P_{s}^{\prime}\right)$ em $\mathcal{V}$ tal que:

i) cada $P_{i}^{\prime}$ intersecta algum $P_{j}$.

ii) $P_{s}^{\prime} \subset P_{r}$.

iii) $s \leq t r$.

segue-se

$$
G(n) \leq H(t n), \forall n \in \mathbb{Z}^{+}
$$

Por outro lado, se $\left(P_{1}^{\prime}, \cdots, P_{s}^{\prime}\right)$ é uma cadeia de placas em $\mathcal{V}$ então existem placas $P_{i}$ em $\mathcal{U}$ tal que $P_{i}^{\prime} \subset P_{i}$, desde que $\mathcal{V}$ é um refinamento de $\mathcal{U},\left(P_{1}, \cdots, P_{r}\right)$ é uma cadeia de placas em $\mathcal{U}$ com $r \leq s$ e segue-se

$$
H(n) \leq t G(n), \forall n \in \mathbb{Z}^{+}
$$

O qual conclui prova no caso que $\mathcal{V}$ é um refinamento de $\mathcal{U}$. No caso geral é suficiente tomar uma cobertura que seja um refinamento de $\mathcal{U}$ e $\mathcal{V}$ e aplicar o caso anterior.

Agora mostraremos o resultado principal de este capítulo, sua demostração é baseada nos tópicos que foram apresentados neste capítulo.

Teorema 2.35 (J. Plante, [17]): Seja $\mathcal{F}$ uma folheação de codimensão $k$ de uma variedade compacta $M$. Se L é uma folha de $\mathcal{F}$ que tem crescimento não exponencial, então existe uma medida não trivial invariante pelo pseudogrupo de holonomia que é finito em conjuntos compactos e que tem suporte contido no fecho de $L$.

Demonstração. Sejam $\mathcal{U}=\left\{U_{\alpha}\right\}_{\alpha=1}^{q}$ e $\mathcal{V}=\left\{V_{\alpha}\right\}_{\alpha=1}^{q}$ dois coberturas regulares de $M$ onde $\psi_{\alpha}: U_{\alpha} \rightarrow D^{n-k} \times D^{k} \subset \mathbb{R}^{n-k} \times \mathbb{R}^{k}$ e $\phi_{\alpha}: V_{\alpha} \rightarrow \mathbb{R}^{n-k} \times \mathbb{R}^{k}$ são as cartas coordenadas para $\mathcal{F}$ com $\overline{U_{\alpha}} \subset V_{\alpha}$ e $\phi_{\alpha}$ é uma extensão de $\psi_{\alpha}$, escolhamos $k$-discos compactos $\triangle_{1}, \cdots, \triangle_{q}$ transversais a $\mathcal{F}$ tal que

$$
\psi_{\alpha}^{-1}\left(\{0\} \times D^{k}\right) \subset \triangle_{i} \subset \phi_{\alpha}^{-1}\left(\{0\} \times D_{2}^{k}\right) \text { para } \alpha=1, \cdots, q
$$

é suficiente tomar $\triangle_{\alpha}=\overline{\psi_{\alpha}^{-1}\left(\{0\} \times D^{k}\right)}$, claramente toda folha de $\mathcal{F}$ intersecta o interior de algum $\triangle_{\alpha}$. Seja $K_{\alpha}$ o espaço de placas da cobertura $\left\{V_{\alpha}\right\}$ que passam por $\triangle_{\alpha}$, então $K_{\alpha}$ é compacto, logo $K=\bigcup_{\alpha=1}^{q} K_{\alpha}$ é um subconjunto compacto do espaço transverso e é 
um subconjunto do espaço transverso gerado por $\mathcal{V}=\left\{V_{\alpha}\right\}_{\alpha=1}^{q}$. Seja $\Gamma$ o pseudogrupo de holonomia gerado por $\mathcal{V}=\left\{V_{\alpha}\right\}_{\alpha=1}^{q}$ e $\Gamma_{K}$ a restrição de $\Gamma$ a $K$.

Se $L$ é uma folha de $\mathcal{F}$ que tem crescimento não exponencial e $P_{0} \in K$ é uma placa em $L$, então $\Gamma_{K}\left(P_{0}\right)$ tem crescimento não exponencial, então pelo Teorema 2.25 existe $\mu_{K}$ uma medida de Borel finita não trivial $\Gamma_{K}$-invariante em $K$, logo pelo Lema $2.31 \mu_{K}$ tem uma única extensão para uma medida $\mu$ em $\Sigma$ que é $\Gamma$-invariante e finita em conjunto compactos e segue-se também que $\operatorname{supp}(\mu) \subset \bar{L}$, pois $\operatorname{supp}\left(\mu_{K}\right) \subset \overline{\Gamma_{K}\left(P_{0}\right)}$.

\subsubsection{Crescimento de folhas desde um ponto de vista geométrico}

A definição de crescimento de uma folha foi feito de uma forma algébrica, mas se $\mathcal{F}$ for $C^{r}$ com $r \geq 1$ existe outra forma de definir o crescimento de uma folha que é puramente geométrica, como se define a continuação.

Uma métrica Riemanniana numa variedade diferenciável $M^{n}$ é uma correspondência que associa a cada ponto $p \in M$ um produto interno em $T_{p} M$ denotado por $<,>_{p}$ ou $g_{p}(.,$.$) , o qual varia diferenciavelmente(continuamente) no seguente sentido.$

Dado $(U, \varphi)$ uma carta coordenada em $M$, associamos a função $g_{i j}^{\varphi}: U \rightarrow \mathbb{R}$ dada por

$$
g_{i j}^{\varphi}(p)=<\frac{\partial}{\partial x_{i}}(p), \frac{\partial}{\partial x_{j}}(p)>_{p}
$$

onde $\frac{\partial}{\partial x_{i}}(p)=D \varphi_{p}^{-1}\left(e_{i}\right)$, com $\left\{e_{i}\right\}_{i}$ a base trivial de $\mathbb{R}^{n}$. Dizemos que a métrica riemanniana $g$ em $M$ é de classe $C^{k}$ se $g_{i j}^{\varphi}$ é $C^{k}$ para cada $(U, \varphi)$. Ou equivalentemente $\forall X, Y \in \chi(V)$ a função $<X(p), Y(p)>_{p}$ é diferenciável em $V$, onde $V$ é uma vizinhança em $M$.

O seguinte resultado é primordial para definir crescimento desde um ponto de vista geométrico, a prova pode ser encontrado em qualquer livro de geometria riemanniana.

Teorema 2.36: Seja $(M, g)$ uma variedade riemanniana orientável, então existe $\omega$ uma única $n$-forma tal que em cada $(U, \varphi)$ coordenada tem a seguente representação

$$
\omega(p)=\sqrt{\operatorname{det}\left(g_{i j}^{\varphi}(p)\right)} d x_{1} \wedge \cdots \wedge d x_{n}, \forall p \in U .
$$

A $n$-forma do Teorema anterior induz o volume. Seja $\left\{\lambda_{\alpha}\right\}$ uma partição da unidade subordinada à cobertura $\left\{U_{\alpha}\right\}$ e $R \subset M$ uma região, definimos o volume de $R$ por

$$
\operatorname{vol}(R)=\sum_{\alpha} \int_{U_{\alpha \cap R}} \lambda_{\alpha} \omega
$$


Definição 2.37: Seja $(M, g)$ uma variedade riemanniana, definimos a função de crescimento de $M$ em $x$ como a função $G_{x}: \mathbb{Z}^{+} \rightarrow \mathbb{R}$ dado por

$$
G_{x}(r)=\operatorname{vol}\left(D_{x}(r)\right)
$$

onde $D_{x}(r)=\{y \in M ; d(x, y) \leq r\}$ e $d$ é a métrica induzida por $g$.

O seguinte resultado mostra que o crescimento de $M$ independente do ponto $x$.

Proposição 2.38: Se $x, y \in M$ então $\operatorname{gr}\left(G_{x}\right)=\operatorname{gr}\left(G_{y}\right)$

Demonstração. Seja $r>d(x, y)$, então $D_{x}(3 r) \supset D_{y}(r)$, pois se $z \in D_{y}(r)$ segue $d(x, z) \leq$ $d(x, y)+d(y, z)<r+r<3 r, \log \mathrm{O}$

$$
G_{x}(3 r) \geq G_{y}(r), \forall r>d(x, y)
$$

assim $G_{x} \succeq G_{y}$, de forma análoga temos que $G_{y} \succeq G_{x}$ o que conluie a prova.

Exemplo 2.39: O plano euclideano $\mathbb{R}^{n}$, com a métrica riemanniana usual tem tipo de crescimento polinomial, pois

$$
\left(\frac{2 r}{\sqrt{n}}\right)^{n} \leq \operatorname{vol}\left(D_{r}(0)\right) \leq(2 r)^{n}
$$

Exemplo 2.40: O plano hiperbólico tem crescimento exponencial, pois, no modelo do disco de Poincare, a metrica esta dada por,

$$
d s^{2}=\frac{d x^{2}+d y^{2}}{\left(1-x^{2}-y^{2}\right)^{2}}
$$

e o elemento volumem é

$$
\sigma=\frac{d x \wedge d y}{\left(1-x^{2}-y^{2}\right)^{2}}
$$

A bola $D_{0}(r)$ é neste caso a bola euclideana de centro 0 e radio $\tanh (r)$ e seu volumem é

$$
\operatorname{vol} D_{0}(r)=\pi(\sinh (r))^{2}=\pi\left(\frac{\exp (-r)-\exp (r)}{2}\right)^{2}
$$

que é do mesmo tipo de crescimento que $\exp (r)$.

A definição 2.37 depende da métrica riemanniana, mas o seguinte resultado mostra que o crescimento de $M$ é independente sobre métricas riemannianas quase-isométricas.

Definição 2.41: Sejam $g$ e $g^{\prime}$ dois métricas Riemannianas em $M$, diz-se que $g$ e $g^{\prime}$ são quase-isométricas se $\exists A, B>0$ tal que 


$$
A\|u\|_{p} \leq\|u\|_{p}^{\prime} \leq B\|u\|_{p}, \forall u, v \in T_{p} M, p \in M
$$

onde \|\|$_{p}$ e \|\|$_{p}^{\prime}$ são as normas induzidas em $T_{p} M$ por $<,>_{p}$ e $<,>_{p}^{\prime}$.

\section{Observação 2.42:}

i) A relação de quase-isometria é uma relação de equivalência.

ii) Se $g$ e $g^{\prime}$ são quase-isométricas, então

$$
A^{n} \sqrt{\operatorname{det}\left(g_{i j}^{\varphi}(p)\right)} \leq \sqrt{\operatorname{det}\left(\left(g_{i j}^{\prime}\right)^{\varphi}(p)\right)} \leq B^{n} \sqrt{\operatorname{det}\left(g_{i j}^{\varphi}(p)\right)}, \forall p \in U
$$

Proposição 2.43: Se g e $g^{\prime}$ são dois métricas riemannianas em $M$ quase-isométricas, então

$$
\operatorname{gr}\left(G_{x}\right)=\operatorname{gr}\left(G_{x}^{\prime}\right)
$$

onde $G_{x}$ e $G_{x}^{\prime}$ são as funções de crescimento de $M$ associado a $g$ e a $g^{\prime}$ respectivamente.

Demonstração. Como $g$ e $g^{\prime}$ são quase-isométricas existem $A, B>0$ tal que

$$
A\|u\| \leq\|u\|^{\prime} \leq B\|u\|, \forall p \in M \text { e } \forall u \in T_{p} M
$$

Afirmação: $D_{x}^{\prime}(A r) \subset D_{x}(r) \subset D_{x}^{\prime}(B r)$. De fato, seja $y \in D_{x}^{\prime}(A r)$ como

$$
d(x, y)=\inf _{\gamma} \int_{0}^{1}\|\dot{\gamma}\| d t
$$

então

$$
A d(x, y)=\inf _{\gamma} \int_{0}^{1} A\|\dot{\gamma}\| d t \leq \inf _{\gamma} \int_{0}^{1}\|\dot{\gamma}\|^{\prime} d t
$$

assim $A d(x, y) \leq d^{\prime}(x, y) \leq A r \Rightarrow D_{x}^{\prime}(A r) \subset D_{x}(r)$ a outra desigualdade é análogo. Sem perda de generalidade vamos supor que $D_{x}^{\prime}(B r) \subset U$ onde $(U, \varphi)$ é uma carta local, da afirmação segue que

$$
A^{n} \operatorname{vol}\left(D_{x}(r)\right)=A^{n} \int_{D_{x}(r)} \sqrt{g_{i j}^{\varphi}} d x_{1} \wedge \cdots \wedge d x_{n} \leq A^{n} \int_{D_{x}^{\prime}(B r)} \sqrt{g_{i j}^{\varphi}} d x_{1} \wedge \cdots \wedge d x_{n}
$$

e pela observação 2.42 temos

$$
A^{n} \int_{D_{x}^{\prime}(B r)} \sqrt{g_{i j}^{\varphi}} d x_{1} \wedge \cdots \wedge d x_{n} \leq \int_{D_{x}^{\prime}(B r)} \sqrt{g_{i j}^{\prime \varphi}} d x_{1} \wedge \cdots \wedge d x_{n}=\operatorname{vol}\left(D_{x}^{\prime}(B r)\right)
$$

então $A^{m} G_{x}(r) \prec G_{n}^{\prime}(B r)$. Assim $G_{x} \preceq G_{x}^{\prime}$. Fazendo a mesmas contas obtemos que $G_{x}^{\prime} \preceq G_{x}$. O que conclui a prova.

Teorema 2.44: Se $M^{n}$ é uma variedade compacta, então todas as métricas riemannianas definidas em $M$ são quase-isométricas. 
Demonstração. Seja $g$ e $g^{\prime}$ dois métricas riemannianas em $M$. Seja $p \in M$, como $T_{p} M \simeq$ $\mathbb{R}^{n}$, então existem $A_{p}, B_{p}>0$ tal que

$$
A_{p}\|u\| \leq\|u\|_{p}^{\prime} \leq B_{p}\|u\|_{p}, u \in T_{p} M
$$

seja $(U, \varphi)$ uma carta local e $X \in \chi(U)$, definimos $f: U \rightarrow \mathbb{R}$ por

$$
f(q)=\sqrt{<X(q), X(q)>_{q}^{\prime}}-A_{p} \sqrt{<X(q), X(q)>_{q}}
$$

como a métrica é $C^{k}$ e $f(p) \geq 0$, então existe $V_{p}$ vizinhança de $p$ tal que $f(q) \geq 0, \forall q \in V_{p}$; i.e.,

$$
\|X(q)\|_{q}^{\prime} \geq A_{p}\|X(q)\|_{q}, \forall q \in V_{p}
$$

Como $X$ é um campo qualquer, seja $q \in V_{p}, v \in T_{p} M$ e $X \in \chi\left(V_{p}\right)$ tal que $X(q)=v$, então

$$
\|v\|_{q}^{\prime} \geq A_{p}\|v\|_{q}, \forall q \in V_{p}, \forall v \in T_{p} M
$$

Como $M$ é compacto, então $M$ pode ser coberto por um número finito de abertos do tipo $V_{p}$; i.e., $M=\bigcup_{i=1}^{m} V_{p_{i}}$. Tomando $A=\min \left\{A_{i}\right\}$, então

$$
\|v\|_{q}^{\prime} \geq A\|v\|_{q}, \forall q \in M, \forall v \in T_{p} M
$$

Da mesma forma se prova a outra desigualdade.

Exemplo 2.45: Uma variedade compacta tem crescimento polinomial de grau zero. Isto é imediato desde que o volumem da variedade seja limitado.

Seja $M$ uma variedade diferenciável compacta de classe $C^{k}(k \geq 1)$ e $\mathcal{F}$ uma folheação em $M$ transversalmente orientável, se $L$ é uma folha de $\mathcal{F}$, então $L$ herda uma métrica riemanniana, como $M$ é compacto então as métricas que $L$ herda são quase-isométricas, então podemos definir a função de crescimento de $L$.

Assim temos dos definições de crescimento de uma folha, desde um ponto de vista geométrico e algebraico. Mas estas dois noções coincidem para folheações suaves, como mostra a seguinte proposição.

Proposição 2.46: Se $L \in \mathcal{F}$ e $P_{0} \in L$, então

$$
\operatorname{gr}(L)=\operatorname{gr}\left(\Gamma\left(P_{0)}\right)\right.
$$

onde $\Gamma=\Gamma_{\mathcal{U}}$ é o pseudogrupo de holonomia. 
Observação 2.47: Antes de fazer a prova da proposição temos que fazer as seguintes observações que são primordiais para a prova.

a) As folhas de $\mathcal{F}$ estão em correspondência biunívoca com as $\Gamma_{\mathcal{U}}$-orbitas no espaço transverso, de fato, dado uma folha $L$ seja $P$ qualquer placa contida em $L$, então associamos a orbita $\Gamma(P)$ a $L$. Por outro lado, dado uma orbita $\Gamma(P)$ associamos a folha $L$ que contem a $P$, verificamos que estas formas de associar são bem definidas e são inversas uma da outra.

Sejam $P_{1}, P_{2} \in T$ tal que $P_{1}, P_{2} \in L$, sabemos que existe $\gamma: \sum_{1} \rightarrow \sum_{2}$ difeomorfismo tal que $\gamma\left(P_{1}\right)=P_{2}$, então $P_{2} \in \Gamma\left(P_{1}\right)$, segue-se $\Gamma\left(P_{1}\right)=\Gamma\left(P_{2}\right)$.

b) Tomemos $0<\epsilon<1$ tal que se $\alpha \neq \beta$ então $\left(P(\epsilon) \times Y_{\alpha}\right) \cap\left(P(\epsilon) \times Y_{\beta}\right)$. Fixemos uma métrica riemanniana em $M$, pela continuidade de esta métrica, a finitude e regularidade de $\left\{U_{\alpha}\right\}_{\alpha}$ e da compacidade de $M$ temos o seguinte.

1. Existe $a>0$ tal que $a \leq \operatorname{vol}_{q}\left(P(\epsilon) \times Y_{\alpha}\right), \forall \epsilon-$ placa em $U_{\alpha}$ e $\forall \alpha$.

2. Existe $b \in \mathbb{Z}^{+}$tal que $\operatorname{vol}_{p}\left(P \times Y_{\alpha}\right) \leq b, \forall P \times Y_{\alpha}$.

3. Existe $B \in \mathbb{Z}^{+}$tal que $\operatorname{diam}\left(P \times Y_{\alpha}\right) \leq B, \forall P \times Y_{\alpha}$.

4. Existe um numero de Lebesgue $\delta>0$, para a cobertura da folha por placas.

c) Se $(M, g)$ uma variedade riemanniana compacta, então $L$ é completa em $g$.

Demostração da Proposição 2.46. Fixe uma placa $P_{0} \in T$, com o correspondente $\epsilon$-placa $P_{0}(\epsilon) \subset P_{0}$ e $x_{0} \in P_{0}(\epsilon)$. Temos que mostrar que a função de crescimento associado a métrica riemanniana $G_{x_{0}}$ de $L$ e a função de crescimento $H_{P_{0}}$ da orbita $\Gamma\left(P_{0}\right)$ são mutuamente dominados.

Seja $\left(P_{0}, P_{1}, \cdots, P_{k}\right)$ uma cadeia de placas $0 \leq k \leq r$ e seja $y \in P_{k}$, por propriedade(3) da observação anterior temos

$$
d(x, y) \leq B r
$$

então da propriedade (1) e de esta ultima desigualdade segue-se

$$
G_{x_{0}}(B r) \geq \sum_{i=0}^{k} \operatorname{vol}\left(P_{i}(\epsilon)\right) \geq(k+1) a
$$

tomando $A \in \mathbb{Z}^{+}$tal que $A \geq \frac{1}{a}$, então

$$
A G_{x_{0}}(B r) \geq(k+1), \forall k \leq r
$$


como $H_{P_{0}}(r)$ é o número de distintas placas que podem ser alcançados por cadeias a partir de $P_{0}$ de comprimento $\leq r$, assim

$$
A G_{x_{0}}(B r) \geq H_{P_{0}}(r) \Rightarrow G_{x_{0}} \succeq H_{P_{0}}
$$

Agora mostraremos que $H_{P_{0}} \succeq G_{x_{0}}$. Fixamos $\eta \in(0, \delta)$, onde $\delta$ é da propriedade (4), seja $L \in \mathcal{F}$ tal que $x_{0} \in L$, tomemos $r \in \mathbb{Z}^{+}$e $y \in L$ tal que $d\left(x_{0}, y\right) \leq r$, pela observação (c) temos que $L$ é completa na métrica riemanniana, então existe uma geodésica minimal $\sigma$ em $L$ de $x_{0}$ até $y$. Escolhamos $x_{0}, x_{1}, \cdots, x_{k}=y$ ao longo de $\sigma$ tal que $\eta<d\left(x_{i}, x_{i-1}\right)<\delta$. Logo pela propriedade (4), existem placas $P_{i} \operatorname{com}\left\{x_{i}, x_{i-1}\right\} \subset P_{i}$ para $1 \leq i \leq k$, então $P_{i} \cap P_{i-1} \neq \emptyset, y \in P_{k}$ e $r \geq d\left(x_{0}, y\right)=\sum_{i=1}^{k} d\left(x_{i}, x_{i-1}>k \eta\right.$. Tomando $D \in \mathbb{Z}^{+}$tal que $D>\frac{1}{\eta}$, então $D r>k$. Como

$$
H_{P_{0}}=\#\left\{\left(P_{0}, P_{1}, \cdots, P_{k}\right) ; k \leq D r\right\}
$$

e $G_{x_{0}}(r)=\operatorname{vol}\left(D_{x_{0}}(r)\right)$, então pelos argumentos anteriores temos que cada ponto de $y \in D_{x_{0}}(r)$ habita em uma placa $P_{k}$ para algum $k$, assim pela propriedade (2) temos

$$
G_{x_{0}}(r) \leq b \#\left\{\left(P_{0}, P_{1}, \cdots, P_{k}\right) ; k \leq D r\right\} \leq b H_{P_{0}}(D r)
$$

de (2.15) e (2.16) fica concluída a prova. 


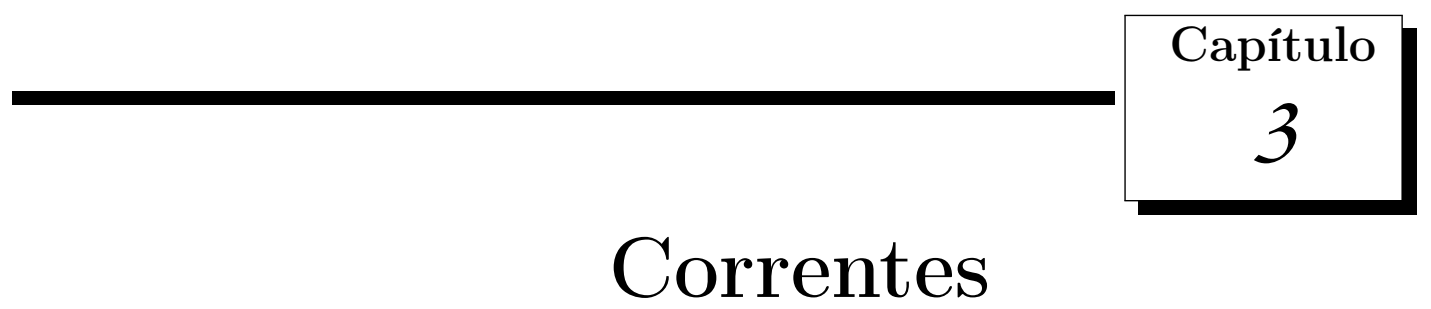

A teoria de correntes de DeRham [8] elaborada nos anos 50 é um análogo da teoria de distribuições de Schwartz [20] criada nos finais de 1940. As distribuições são funcionais contínuas definidas no espaço das funções com suporte compacto quando se dota a dito espaço com uma topologia adequada. Da mesma maneira, quando se dota com uma topologia conveniente ao espaço das formas diferenciais com suporte compacto $\Omega_{c}^{p}$, as $p$-correntes se definem como funcionais contínuos definidos em $\Omega_{c}^{p}$; e.g., as distribuições são 0-correntes. Além disso, dita topologia faz que a derivada exterior seja um operador linear continuo, pelo que podemos definir seu operador adjunto e a partir de este definir a homologia de DeRham. Neste capítulo o resultado principal é mostrar o teorema de D. Sullivan [23] que diz que as medidas transversas invariantes por holonomia estão em correspondência canônica um a um com ciclos folheados(um tipo especial de correntes). Este capítulo em sua maioria foi extraído e estudado do livro [7] e [8].

\subsection{Correntes}

Ao longo de esta seção $M$ será uma variedade n-dimensional de classe $C^{\infty}$. Introduziremos uma topologia no espaço $\Omega^{p, r}:=\Omega^{p, r}(M)\left(\operatorname{resp} . \Omega^{p}:=\Omega^{p}(M)\right)$ das $p$-formas diferenciáveis de classe $C^{r}$ (resp. de classe $C^{\infty}$ ) sobre $M$.

Tomemos $U \subset M$ uma aberto coordenado. Para cada $\omega \in \Omega^{p, r}$ podemos escrever, em coordenadas locais, $\omega(x)=\sum_{|I|=p} \omega_{I}(x) d x_{I}$ para todo $x \in U$, onde $I=\left(i_{1}, \cdots, i_{p}\right)$ é um multi-índice com $i_{1}<\cdots<i_{p}, i_{1}, \cdots, i_{p} \in\{1,2, \cdots, n\}$. Logo, para qualquer $r \in \mathbb{N} \cup\{0\}$ 
e qualquer $K \subset U$ compacto definimos a seminorma $\|\cdot\|_{K}^{r}$ por

$$
\|\omega\|_{K}^{r}=\sup _{x \in k} \sum_{|\alpha| \leq r,|I|=p}\left|D^{\alpha} \omega_{I}(x)\right|
$$

onde $\alpha=\left(\alpha_{1}, \cdots, \alpha_{n}\right) \in\left(\mathbb{Z}^{+}\right)^{n}$ e $D^{\alpha}=\frac{\partial^{|\alpha|}}{\partial_{x_{1}}^{\alpha_{1}} \cdots \partial_{x_{n}}^{\alpha_{n}}}$ é a derivada de ordem $|\alpha|=$ $\alpha_{1}+\cdots+\alpha_{n}$.

A família de seminormas $\left\{\|\cdot\|_{K}^{r}\right\}_{K, U}$ é separador(ver apêndice $A$ ), por tanto geram uma topologia $\mathcal{T}^{r}$ em $\Omega^{p, r}$ e que faz de $\Omega^{p, r}$ um espaço topológico de Hausdorff localmente convexo. Agora, notemos que $\mathcal{T}^{r} \subset \mathcal{T}^{r+1}$, então $\bigcup_{r \geq 0} \mathcal{T}^{r}$ geram uma topologia $\mathcal{T}$ para o espaço $\Omega^{p}$ que é a topologia definida pelas seminormas de 3.1 quando $U, K$ e $r$ variam. É claro que $\Omega^{p}$ com esta topologia também é um espaço de Hausdorff localmente convexo.

\section{Definição 3.1:}

a) Definimos o espaço $\mathcal{E}_{p}:=\mathcal{E}_{p}(M)\left(\operatorname{resp} . \mathcal{E}_{p}^{r}:=\mathcal{E}_{p}^{r}(M)\right)$ como o espaço $\Omega^{p}\left(\right.$ resp. $\left.\Omega^{p, r}\right)$ com a topologia $\mathcal{T}$ (resp. $\left.\mathcal{T}^{r}\right)$.

b) Se $K \subset M$ é um compacto, definimos

$$
\mathcal{D}_{p}(K):=\left\{\omega \in \mathcal{E}_{p}: \operatorname{supp}(\omega) \subset K\right\}
$$

com a topologia induzida de $\mathcal{E}_{p} . \mathcal{D}_{p}:=\mathcal{D}_{p}(M):=\bigcup_{K} \mathcal{D}_{p}(K)$, é dizer todas as $p$-formas com suporte compacto. Os espaços $\mathcal{D}_{p}^{r}(K)$ e $\mathcal{D}_{p}^{r}:=\mathcal{D}_{p}^{r}(M)$ se definem de maneira similar.

Lema 3.2: Dado um conjunto compacto $K \subset M$, uma sequencia $\left\{\omega_{i}\right\}$ converge a $\omega \in$ $\mathcal{D}_{p}(K)$ se, e somente se, converge a $\omega \in \mathcal{D}_{p}^{r}(K)$ para todo $r \in \mathbb{Z}^{+}$.

Demonstração. Desde que $\mathcal{T}^{r} \subset \mathcal{T}$, convergência em $\mathcal{D}_{p}(K)$ implica convergência em $\mathcal{D}_{p}^{r}(K)$. Reciprocamente, cada vizinhança aberta $W$ de $\omega$ em $\mathcal{D}_{p}(K)$ contem uma vizinhança de $\omega$ da forma $W_{j_{1}} \cap \cdots \cap W_{j_{q}}$, para algum inteiro $q \geq 1$, onde $W_{j_{i}}$ é uma vizinhança aberta de $\omega$ em $\mathcal{D}_{p}^{j_{i}}(K)$. A convergência em cada $\mathcal{D}_{p}^{r}(K)$ implica que os $\omega_{i}$ 's excepto um numero finito estão em $W_{j_{1}} \cap \cdots \cap W_{j_{q}}$, assim em $W$. Desde que $W$ foi vizinhança arbitraria de $\omega$ segue que $\omega_{i} \rightarrow \omega$ em $D_{p}(K)$.

Observação 3.3: Uma sequencia $\left\{\omega_{i}\right\}_{i}$ converge a $\omega \in \mathcal{D}_{p}$ se, e somente se, $\left\{\omega_{i}\right\}_{i}$ esta contida em $\mathcal{D}_{p}(K)$ para algum $K \subset M$ compacto tal que $\left\{\omega_{i}\right\}_{i}$ converge a $\omega$ em $\mathcal{D}_{p}(K)$. 
Lema 3.4: Um funcional $C: \mathcal{D}_{p} \rightarrow \mathcal{D}_{q}$ (resp. $\left.C: \mathcal{D}_{p} \rightarrow \mathbb{R}\right)$ é continuo se, e somente se, $\left\{C\left(\omega_{i}\right)\right\}_{i \in \mathbb{N}} \subset \mathcal{D}_{p}$ converge a $C(\omega)$ em $\mathcal{D}_{q}$ (resp. em $\mathbb{R}$ ) para toda sequencia $\left\{\omega_{i}\right\}_{i \in \mathbb{N}} \subset \mathcal{D}_{p}$ converge $a \omega \in \mathcal{D}_{p}$.

Demonstração. Vamos supor que $C: \mathcal{D}_{p} \rightarrow \mathcal{D}_{q}$ que não es continua em $\omega \in D_{p}$, existe uma vizinhança $V$ de $C(\omega)$ em $\mathcal{D}_{q}$ tal que $C(W) \nsubseteq V$ para toda vizinhança $W$ de $\omega$ em $\mathcal{D}_{p}$. Em particular, seja $W_{r} \in \mathcal{T}^{r} \subset \mathcal{T}$ as bolas centradas em $\omega$ de radio $\frac{1}{r}$ na norma $\|\cdot\|_{K}^{r}$ e escolhemos $\omega_{r} \in W_{r}$ tal que $C\left(\omega_{r}\right) \notin V, \forall r \geq 0$. Desde que

$$
\left\|\omega_{r}-\omega\right\|_{K}^{0} \leq\left\|\omega_{r}-\omega\right\|_{K}^{1} \leq \cdots\left\|\omega_{r}-\omega\right\|_{K}^{r} \leq \frac{1}{r}, \forall r \geq 0
$$

entao, $\omega_{r} \rightarrow \omega$ em $D_{p}^{r}, \forall r \geq 0$ e pelo Lema 3.2 temos que $\omega_{r} \rightarrow \omega$ em $D_{p}$, o qual é uma contradição. O reciproco é imediato, pois toda aplicação continua é sequencialmente continua.

Proposição 3.5: Um funcional linear $C: \mathcal{D}_{p} \rightarrow \mathbb{R}$ é continuo se, e somente se, para cada compacto $K \subset M$ existem $c>0$ e $r \in \mathbb{Z}^{+}$tais que

$$
|<C, \omega>| \leq c\|\omega\|_{K}^{r}, \forall \omega \in \mathcal{D}_{p}(K)
$$

onde $|\langle C, \omega\rangle|$ denota a avaliação de $C$ na forma $\omega$.

Demonstração. Segue imediatamente do Teorema A.3.

Definição 3.6: Seja $S \subset \mathcal{D}_{p}$ dizemos que $S$ é limitado, se é limitado relativo à norma $\|\cdot\|_{K}^{r}$ para todo $r \in \mathbb{Z}^{+}$e todo compacto $K \subset M$. Da mesma maneira, uma aplicação linear $C$ de $\mathcal{D}_{p}$ a $\mathcal{D}_{q}$ (resp. a $\mathbb{R}$ ) se diz que é limitada, se é limitada com respeito à norma $\|\cdot\|_{K}^{r}$, isto é, se para toda sequencia limitada em $\mathcal{D}_{p}$, a imagem da sequencia sobre $C$ é uma sequencia limitada em $\mathcal{D}_{q}$ (resp. em $\mathbb{R}$ ).

Lema 3.7: Uma aplicação linear limitado $C: \mathcal{D}_{p} \rightarrow \mathcal{D}_{q}$ é continua. Uma aplicação linear $C: \mathcal{D}_{p-1} \rightarrow \mathbb{R}$ é continua se e somente se é limitada.

Demonstração. Se $C$ é uma aplicação linear limitado; i.e., limitado relativo às normas \|\|$_{K}^{r}, \forall r, K$. Logo $C$ é continua com respeito a estas normas, assim $C$ é sequencialmente contínua na topologia $\mathcal{T}^{r}, \forall r \geq 0$. Logo pelo Lema 3.3 temos que $C$ é sequencialmente continua na topologia $\mathcal{T}$ e pelo Lema 3.4 segue que $C$ é contínua. Reciprocamente, se $C$ não é limitado, existe uma sequencia $\left\{\omega_{k}\right\}_{k}$ em $\mathcal{D}_{p}$ limitado tal que $\left\langle C, \omega_{k}\right\rangle \geq k$, $1 \leq k<\infty$. Tomemos a sequencia $\left\{\lambda_{k}=\frac{\omega_{k}}{k}\right\}_{k}$, desde que $\left\{\omega_{k}\right\}_{k}$ é limitada temos 
$\lim \lambda_{k}=0$ na topologia $\mathcal{T}$. Por outro lado temos que $\left\langle C, \lambda_{k}\right\rangle \geq 1$, e pelo Lema 3.4 segue que $C$ não é continua.

A derivada exterior $d: \mathcal{D}_{p-1} \rightarrow \mathcal{D}_{p}$ é limitada, pois os elementos de $\mathcal{D}_{p}$ são formas com suporte compacto cujos coeficientes são de classe $C^{\infty}$, então a imagem de uma sequencia limitada sobre $d$ é limitada. Assim temos o seguente corolário.

Corolário 3.8: A derivada exterior $d: \mathcal{D}_{p-1} \rightarrow \mathcal{D}_{p}$ é uma aplicação linear continua.

Agora já podemos definir o espaço das p-correntes.

Definição 3.9: Definimos o espaço das p-correntes em $M$ como o espaço das aplicações lineares de $\mathcal{D}_{p}$ a $\mathbb{R}$, tais que sua restrição a $\mathcal{D}_{p}(K)$ é continua para todo $K \subset M$ compacto; i.e.,

$$
\mathcal{D}_{p}^{\prime}=\left\{C: \mathcal{D}_{p} \rightarrow \mathbb{R}: C \text { é linear e }\left.C\right|_{\mathcal{D}_{p}(K)} \text { é continuo } \forall K \subset M \text { compacto }\right\}
$$

Notemos que podemos definir uma corrente de maneira equivalente como uma função continua $C: \mathcal{D}_{p} \rightarrow \mathbb{R}$ que satisfaz (3.2).

\section{Observação 3.10:}

i) As correntes são uma generalização das distribuições, neste caso uma distribuição é um 0-corrente.

ii) Dado $C$ uma $n$-corrente, podemos associar a ela uma distribuição $T$, da seguinte maneira

$$
\langle T, f\rangle=\left\langle C, f d x_{1} \wedge \cdots \wedge d x_{n}\right\rangle, f \in C_{c}^{\infty}(M)
$$

Exemplo 3.11: Seja $x \in M$ e $v_{x} \in \Lambda_{p}\left(T_{x} M\right)$, definimos o funcional $C_{v_{x}}$ por

$$
<C_{v_{x}}, \omega>:=\omega\left(v_{x}\right), \forall \omega \in \mathcal{D}_{p}
$$

este funcional linear define uma $p$-corrente de ordem 0 chamada corrente de Dirac.

Exemplo 3.12 (Corrente de integração): Suponhamos que $M$ é compacta e seja $\mu$ uma medida de probabilidade definida em $M$. Consideremos um campo continuo de $p$-vetores $X$ em $M$. definimos $C_{X, \mu}$ por

$$
<C_{X, \mu}, \omega>:=\int_{M} \omega(X) d \mu, \forall \omega \in \mathcal{D}_{p}
$$

este funcional linear define uma p-corrente chamada corrente de integração. 
Exemplo 3.13: Seja $Z \subset M$ uma subvariedade fechada e orientável de $M$ de dimensão $p$ e classe $C^{1}$. Definimos o funcional linear $C_{Z}$ por

$$
<C_{Z}, \omega>:=\int_{Z} \omega
$$

que define uma $p$-corrente de ordem 0.

Exemplo 3.14: Suponhamos que $M$ é orientável e seja $\eta$ uma $(n-p)$-forma, definimos $C \eta$ por

$$
<C_{\eta}, \omega>:=\int_{M} \omega \wedge \eta, \forall \omega \in \mathcal{D}_{p}
$$

este funcional linear define uma $p$-corrente chamada corrente difusa.

O seguinte é um exemplo importante que faz a ligação das medidas transversas invariantes que foram estudadas no capítulo 2 com as correntes; para ser mais específicos com "ciclos folheados"que serão estudadas na seguinte seção.

Exemplo 3.15 (corrente associada a uma medida transversa invariante): Seja $\mathcal{F}$ uma folheação $p$-dimensional em $M$ variedade diferenciável compacta. Seja $\mathcal{U}=\left\{U_{\alpha}\right\}_{\alpha \in I}$ um atlas regular folheado, $\Sigma=\bigsqcup_{\alpha} \Sigma_{\alpha}$ o espaço transverso, $\gamma_{\alpha \beta}: \Sigma_{\alpha} \rightarrow \Sigma_{\beta}$ os difeomorfismos locais, $\pi_{\alpha}: U_{\alpha} \rightarrow \Sigma_{\alpha}$ a projeção canônica para cada $\alpha \in I$ e $\Gamma=\Gamma_{\mathcal{U}}$ o pseudogrupo de holonomia gerado por $\Gamma_{1}=\left\{\gamma_{\alpha \beta} ; \alpha, \beta \in I\right\}$. Seja $\mu$ uma medida em $\Sigma$ transversa invariante por holonomia. Então, para qualquer $U_{\alpha} \in \mathcal{U}$, podemos integrar uma $p$-forma $\omega$ sobre cada placa $\pi_{\alpha}^{-1}(x)$. Isto define uma função continua de $\Sigma_{\alpha}$ a $\mathbb{R}$, que ao ser integrada com respeito à medida $\left.\mu\right|_{\Sigma_{\alpha}}$ se obtêm uma aplicação linear real $C_{\mu}$ do conjunto das $p$-formas

$$
<C_{\mu}, \omega>:=\sum_{\alpha \in I} \int_{\Sigma_{\alpha}}\left(\int_{\pi_{\alpha}^{-1}(x)} \lambda_{\alpha} \omega\right) d \mu(x)
$$

Na seção 1 , do capítulo 2 , foi provado que a definição de $C_{\mu}$ não depende da partição da unidade escolhida e da cobertura regular folheada. Assim $C_{\mu}$ define uma $p$-corrente.

Seja $C \in \mathcal{D}_{p}^{\prime}$ e $\beta \in \mathcal{D}_{q}$ com $q \leq p$. Definamos o produto wedge $C \wedge \beta$ por

$$
\langle C \wedge \beta, \omega\rangle=\langle C, \beta \wedge \omega\rangle, \omega \in \mathcal{D}_{p-q}
$$

i.e., que $C \wedge \beta$ é uma $(p-q)$-corrente. Definimos $\beta \wedge C=(-1)^{p-q} C \wedge \beta$.

O seguinte resultado diz que as correntes, podem ser pensados como formas diferenciais com coeficientes as distribuições. A prova de este resultado pode ser consultada em [8]. 
Teorema 3.16: Seja $C$ uma p-corrente em $M^{n}$. Então $C$ pode ser escrito em uma única forma

$$
C=\sum_{|I|=n-p} C_{I} \wedge d x_{I}
$$

onde os $C_{I}$ são n-correntes em $M$.

Definição 3.17: $\quad$ O suporte de uma $p$-corrente $C$ é o menor subconjunto fechado $\operatorname{supp}(C)$ de $M$ tal que $C$ se anula em $M \backslash \operatorname{supp}(C)$. Isto é, $\langle C, \omega\rangle=0$ para todo $\omega \in \Omega^{p}$ com suporte em $M \backslash \operatorname{supp} C$.

Sejam $M, N$ duas variedades, $C$ um $p$-corrente em $M$ e $f: M \rightarrow N$ uma aplicação de classe $C^{1}$ que é própria no suporte de $C$.

Definição 3.18: Definimos o push-forward de $C$ por $f$ denotado $f_{*} C$ como

$$
\left\langle f_{*} C, \omega\right\rangle=\left\langle C, f^{*} \omega\right\rangle, \forall \omega \in \mathcal{D}_{p}(N)
$$

onde $f^{*} \omega$ é o pull-back de $\omega$ por $f$. Assim definido $f_{*} C$ é uma $p$-corrente em $N$.

A continuação dotaremos de uma topologia ao espaço das correntes e definiremos a homologia de DeRham.

Por definição, o espaço das $p$-correntes é o dual continuo do espaço $\mathcal{D}_{p}$, o qual permite definir uma topologia $\mathcal{T}^{\prime}$ em $\mathcal{D}_{p}^{\prime}$ que o converte em um espaço de Hausdorff localmente convexo da maneira seguinte.

Para $\epsilon>0$ e $B \subset \mathcal{D}_{p}$ limitado, definimos os conjuntos

$$
V_{B, \epsilon}=\left\{C \in \mathcal{D}_{p}^{\prime} ;<C, B>\subset[-\epsilon, \epsilon]\right\}
$$

Um subconjunto $S_{0} \subset \mathcal{D}_{p}^{\prime}$ é uma vizinhança de 0 se $V_{B, \epsilon} \subset S_{0}$ para algum $B$ e $\epsilon>0$. Se $C \in \mathcal{D}_{p}^{\prime}$ a vizinhança de $C$ na topologia $\mathcal{T}^{\prime}$ sao os conjuntos da forma $S_{C}=C+S_{0}$. Um subconjunto $W \subset \mathcal{D}_{p}^{\prime}$ é aberto se, para cada $C \in W$, existe uma vizinhança $S_{C} \subset W$.

Proposição 3.19: Sejam $\left\{C_{n}\right\}_{n}$ uma sequencia em $D_{p}^{\prime}$ e $C \in D_{p}^{\prime}$. Então,

$$
C_{n} \rightarrow C \Leftrightarrow\left\langle C_{n}, \omega\right\rangle \rightarrow\langle C, \omega\rangle, \forall \omega \in \mathcal{D}_{p}
$$

Demonstração. Sem perda de generalidade podemos supor que $C=0$. Seja $\omega \in \mathcal{D}_{p}$ qualquer e $\epsilon>0$. Logo, $V_{B, \epsilon}$ é uma vizinhança de 0 com $B=\{\omega\}$. Como $C_{n} \rightarrow 0$, então existe $n_{0} \in \mathbb{N}$ tal que $C_{n} \in V_{B, \epsilon}$ para todo $n \geq n_{0}$, assim 


$$
\left|\left\langle C_{n}, \omega\right\rangle\right|<\epsilon, \text { para } n \geq n_{0}
$$

Por tanto, $\left\langle C_{n}, \omega\right\rangle \rightarrow 0$.

Definição 3.20: Um conjunto $V \subset \mathcal{D}_{p}^{\prime}$ é limitado, se para cada $B \subset \mathcal{D}_{p}$ limitado o conjunto $\{\langle C, \omega\rangle ; C \in V, \omega \in B\} \subset \mathbb{R}$ é limitado.

Consideremos a aplicação bilinear

$$
e: \mathcal{D}_{p}^{\prime} \times \mathcal{D}_{p} \rightarrow \mathbb{R}
$$

definido por $e(C, \omega):=<C, \omega>$. Esta aplicação permite identificar $\mathcal{D}_{p}$ com o espaço $\mathcal{D}_{p}^{\prime \prime}$ de funcionais lineares contínuos sobre $\mathcal{D}_{p}^{\prime}$. i.e, $\mathcal{D}_{p}$ é identificado com seu doble dual $\mathcal{D}_{p}^{\prime \prime}$ a prova de isto pode ser encontrada em [8].

Desde que a derivada exterior $d: \mathcal{D}_{p-1} \rightarrow \mathcal{D}_{p}$ é um operador limitado podemos definir seu operador adjunto.

Definição 3.21: Definimos o operador $\partial:=\partial_{p}: \mathcal{D}_{p}^{\prime} \rightarrow \mathcal{D}_{p-1}^{\prime}$ por

$$
<\partial C, \omega>:=<C, d \omega>, \forall \omega \in \mathcal{D}_{p-1}, C \in \mathcal{D}_{p}^{\prime}
$$

i.e., $\partial$ é o operador adjunto de $d$.

Notemos que $\partial^{2}=0$, pois para qualquer $C \in \mathcal{D}_{p}^{\prime}$ e $\omega \in \mathcal{D}_{p}$ tem-se

$$
<\partial^{2} C, \omega>=<\partial C, d \omega>=<C, d^{2} \omega>=0
$$

Assim podemos definir a $p$-ésima homologia de DeRham de $M$ como segue.

Definição 3.22: Definimos o espaço de $p$-correntes fechadas ou $p$-ciclos e o espaço de $p$-correntes exatas ou $p$-fronteiras, respectivamente, como

$$
\mathscr{Z}_{p}:=\operatorname{Ker} \partial_{p} \text { e } \mathscr{B}_{p}:=\operatorname{Im} \partial_{p+1}
$$

A p-ésima homologia de Rham fica definida então como o espaço

$$
H_{p}^{D R}(M):=\frac{\mathscr{Z}_{p}}{\mathscr{B}_{p}}
$$


A aplicação

$$
\begin{array}{cl}
H_{p}^{D R}(M) \times H_{c}^{p}(M) & \longrightarrow \mathbb{R} \\
([C],[\omega]) & \mapsto\langle C, \omega\rangle
\end{array}
$$

induz o seguente isomorfismo(ver [8] $H_{p}^{D R}(M) \simeq\left(H_{c}^{p}(M)\right)^{*}$. Logo pelo Lema de Poincaré, temos

$$
H_{p}^{D R}(M) \simeq\left(H_{c}^{p}(M)\right)^{*} \simeq H^{n-p}(M)
$$

Exemplo 3.23: Seja $M$ compacto, orientável e sem bordo, definimos a $p$-corrente $C_{M}$ : $\mathcal{D}_{p} \rightarrow \mathbb{R}$ por

$$
\left\langle C_{M}, \omega\right\rangle=\int_{M} \omega
$$

Afirmacao: $H_{n}^{D R} \simeq<C_{M}>$. De fato, como

$$
\mathcal{D}_{n-1} \stackrel{d_{n-1}}{\longrightarrow} \mathcal{D}_{n} \stackrel{d_{n}}{\longrightarrow} 0 \Rightarrow 0 \stackrel{\partial_{n+1}}{\longrightarrow} \mathcal{D}_{n}^{\prime} \stackrel{\partial_{n}}{\longrightarrow} \mathcal{D}_{n-1}^{\prime}
$$

Logo, $\mathscr{B}_{n}=\{0\}$. Por outro lado, pela definição do operador adjunto e Teorema de Stokes temos que

$$
\left\langle\partial C_{M}, \omega\right\rangle=\left\langle C_{M}, d \omega\right\rangle=\int_{M} d \omega=0, \forall \omega \in \mathcal{D}_{p}
$$

$\operatorname{assim} C_{M} \in \mathscr{Z}_{n}$. Logo, $H_{p}^{D R}(M)=\frac{\mathscr{Z}_{p}}{\mathscr{B}_{p}}=\mathscr{Z}_{p}$. Como $H^{0}(M) \simeq \mathbb{R}$ segue de (3.9) que $\operatorname{dim}\left(H_{n}^{D R}\right)=1$, o que prova a afirmação.

\subsection{Corrente Folheada e ciclos folheados}

Nesta seção definiremos a corrente folheada e ciclos folheados. Provaremos que as correntes folheadas são correntes de integração como foi definido no exemplo 3.14, ainda mais, mostraremos que as correntes folheadas estão em correspondência biunívoca com as medidas limitadas e não negativas em $M$.

Daqui em diante consideremos uma variedade folheada $(M, \mathcal{F})$, onde $M$ é uma variedade $C^{\infty}$ compacta $n$-dimensional sem fronteira e $\mathcal{F}$ é uma folheação de dimensão $p$, transversalmente orientável e de classe $C^{1}$.

Tomemos $x \in M$ e seja $L \subset M$ a folha de $\mathcal{F}$ que contem a $x$. Consideremos o $p$-vetor

$$
v_{x}=v_{1 x} \wedge \cdots \wedge v_{p x} \in \Lambda^{p}\left(T_{x} L\right)
$$

onde $\left\{v_{1 x}, \cdots, v_{p x}\right\}$ é uma base ordenada de $T_{x} L$ que dá a correta orientação; i.e., coincidem com a orientação de $T_{x} L$. Denotamos por $C_{v_{x}}$ a sua corrente de Dirac associada(ver exemplo 3.11). 


\section{Definição 3.24:}

a) Definimos o conjunto das correntes folheadas de $\operatorname{Dirac}($ de $\mathcal{F})$ como o conjunto $\left\{C_{v_{x}} ; x \in\right.$ $M\}$.

b) Uma $p$-forma $\omega \in \mathcal{D}_{p}$ se diz transversal a $\mathcal{F}$, se $\left\langle C_{v_{x}}, \omega\right\rangle=\omega\left(C_{v_{x}}\right)>0$ para cada $v_{x} \neq 0$, $x \in M$.

O seguinte lema nos diz que sempre existe $\omega \in \mathcal{D}_{p}$ transversal a $\mathcal{F}$.

Lema 3.25: Uma folheação admite formas transversais.

Demonstração. Seja $\mathcal{U}=\left\{U_{\alpha}\right\}_{\alpha \in I}$ um atlas regular folheado associado a $\mathcal{F}$. Para cada $\alpha \in I$ definimos $\omega_{\alpha}=d x_{1}^{\alpha} \wedge \cdots \wedge d x_{p}^{\alpha}$, onde o vetor coordenado $\left(x_{1}^{\alpha}, \cdots, x_{p}^{\alpha}\right)$ coincide com a orientação de $\left.\mathcal{F}\right|_{U}$. Logo $\omega_{\alpha}\left(v_{x}\right)=1$ onde $v_{x}=\left(x_{1}^{\alpha}, \cdots, x_{p}^{\alpha}\right)$. Seja $\left\{\lambda_{\alpha}\right\}_{\alpha \in I}$ uma partição da unidade subordinada a $\mathcal{U}$, então $\omega=\sum \lambda_{\alpha} \omega_{\alpha}$ é uma $p$-forma transversal.

\section{Definição 3.26:}

a) Denotemos por $\mathcal{C}_{\mathcal{F}}$ o fecho topológico em $\mathcal{D}_{p}^{\prime}$ das combinações lineares finitas com coeficientes positivos das correntes folheadas de Dirac; i.e.,

$$
\mathcal{C}_{\mathcal{F}}=\overline{\left\{\sum_{i \in I} a_{1} C_{v_{x_{i}}} ; I \text { finito, } a_{i}>0\right\}} \mathcal{D}_{p}^{\prime}
$$

seus elementos são chamados correntes folheadas.

b) Definimos os conjuntos

$$
\mathcal{Z}_{\mathcal{F}}:=\mathcal{C}_{\mathcal{F}} \cap \mathcal{Z}_{p} \quad \text { e } \quad \mathcal{B}_{\mathcal{F}}:=\mathcal{C}_{\mathcal{F}} \cap \mathcal{B}_{p}
$$

e são chamados ciclos folheados e de fronteiras folheadas respectivamente.

Demonstraremos agora que as correntes folheadas são correntes de integração como foi definido no exemplo 3.12 pela expressão (3.4). Isto é, demonstraremos que a uma corrente folheada podemos designar uma única medida limitada não negativa.

Seja $\omega$ uma $p$-forma transversal a $\mathcal{F}$ e $X$ um campo continuo de $p$-vetores tangentes a $\mathcal{F}$ tal que $\omega(X) \equiv 1$. Dada $C \in \mathcal{C}_{\mathcal{F}}$ definimos o funcional linear continuo $J_{C}: C^{\infty}(M, \mathbb{R}) \rightarrow \mathbb{R}$ por

$$
J_{C}(f)=\langle C, f \omega\rangle
$$


O seguinte lema diz que podemos associar uma única medida limitada não negativa a este funcional, dita medida será a que associamos a uma corrente folheada.

Lema 3.27: Existe uma única medida $\nu_{C}$ limitada e não negativa sobre $M$, tal que

$$
J_{C}(f)=\int_{M} f d \nu_{C}, \forall f \in C^{\infty}(M, \mathbb{R})
$$

Demonstração. mostraremos a existência de $\nu_{C}$.

caso i: Se $C=C_{X(x)}$ é uma corrente de Dirac.

$$
\begin{aligned}
J_{C}(f) & =\langle C, f \omega\rangle=\left\langle C_{X_{x}}, f \omega\right\rangle \\
& =(f \omega)_{x}(X(x))=f(x) \omega_{x}(X(x)) \\
& =f(x)=\int_{M} f(x) d \nu_{C}
\end{aligned}
$$

onde $\nu_{C}$ é a medida de Dirac em $x$.

caso ii: $C=C_{n}=\sum_{i=1}^{n} a_{i} C_{X\left(x_{i}\right)}, a_{i}>0$. Pelo caso i, é suficiente tomar a medida

$$
\nu_{C}=\sum_{i=1}^{n} a_{i} \nu_{C_{i}}
$$

caso iii: Se $C=\lim _{n \rightarrow \infty} C_{n}$, onde $C_{n}$ é como no caso ii. Para cada $C_{n}$ existe $\nu_{C_{n}}$ medida limitada e não negativa tal que

$$
\left\langle C_{n}, f \omega\right\rangle J_{C_{n}}(f)=\int_{M} f d \nu_{C_{n}}, \forall f \in C^{\infty}(M, \mathbb{R})
$$

desde que $C_{n} \rightarrow C$ temos que $\left\{\nu_{C_{n}}\right\}_{n}$ é uma sequencia de medidas limitadas, então contém uma subsequencia $\left\{\nu_{C_{n}}\right\}_{n_{k}}$ convergente na topologia fraca*; i.e.,

$$
\exists \nu_{C} \text { medida em } M \text { tal que } \nu_{C_{n_{k}}} \stackrel{*}{\rightarrow} \nu_{C} \text { quando } k \rightarrow \infty
$$

por definição de convergência na topologia fraca* temos que

$$
\lim _{k \rightarrow \infty} \int_{M} f d \nu_{C_{n_{k}}}=\int_{M} f d \nu_{C}, \forall f \in C^{\infty}(M, \mathbb{R})
$$

como $C_{n} \rightarrow C$ por Proposição 3.19 segue $\left\langle C_{n}, f \omega\right\rangle \rightarrow\langle C, f \omega\rangle$. Tomando limite em 3.13, segue o Lema de 3.14. Agora mostraremos a unicidade. Suponha que existe $\nu_{C}^{\prime}$ tal que satisfaz 3.12. Logo, como $C^{\infty}(M)$ é denso em $C^{0}(M)$, então

$$
\int_{M} f d \nu_{C}^{\prime}=\int_{M} f d \nu_{C}, \forall f \in C^{0}(M)
$$

segue-se $\nu_{C}^{\prime}=\nu_{C}$. 
Dado $C \in \mathcal{C}_{\mathcal{F}}$ uma corrente folheada pelo Lema anterior mostramos que existe uma única medida satisfazendo (3.12). Assim podemos associar a esta medida uma corrente de integração $C_{X, \nu_{C}}$ que foi definida como,

$$
\left\langle C_{X, \nu_{C}}, \eta\right\rangle=\int_{M} \eta(X) d \nu_{C}, \eta \in \mathcal{D}_{p}
$$

Proposição 3.28: Seja $X$ um campo de p-vetores continuo e sem singularidades tangentes a $\mathcal{F}$. Então as correntes folheadas se correspondem um a um com as medidas limitadas e não negativas sobre $M$ por

$$
C=C_{X, \nu_{C}}
$$

onde $\nu_{C}$ é a medida dada pelo lema anterior.

Demonstração. Provaremos que $C=C_{X, \nu_{C}}$. Seja $C=\lim _{n \rightarrow \infty} C_{n}$ e $\nu_{C}, \nu_{C_{n}}$ como na prova do Lema anterior; i.e., $\nu_{c_{n_{k}}} \stackrel{*}{\rightarrow} \nu_{C}$, então

$$
\lim _{k \rightarrow \infty} \int_{M} f d \nu_{C_{n_{k}}}=\int_{M} f d \nu_{C}, \forall f \in C^{0}(M, \mathbb{R})
$$

Afirmação 1: Dado $\eta \in \mathcal{D}_{p} \Rightarrow \eta=\eta_{0}+\eta(X) \omega$ onde $\eta_{0}(X) \equiv 0$. De fato, definamos $\eta_{0}=\eta-\eta(X) \omega$, desde que $\omega(X) \equiv 1$, temos $\eta_{0}(X(x))=\eta(X(x))-\eta(X(x)) \omega(X(x))=0$. Por outro lado, do Lema anterior

$$
\left\langle J_{C_{n}}, \eta(X)\right\rangle=\int_{M} \eta(X) d \nu_{C_{n}}, n \geq 1
$$

$\operatorname{mas}\left\langle J_{C_{n}}, \eta(X)\right\rangle=\left\langle C_{n}, \eta(X) \omega\right\rangle$ e da afirmação 1 segue $\left\langle J_{C_{n}}, \eta(X)\right\rangle=\left\langle C_{n}, \eta(X)-\eta_{0}\right\rangle$. Desde que $C_{n} \rightarrow C$, por (3.15) e a definição de corrente de integração temos

$$
\left\langle C_{n}, \eta(X)-\eta_{0}\right\rangle=\int_{M} \eta(X) d \nu_{C}=\left\langle C_{X, \nu_{C}}, \eta\right\rangle
$$

Afirmação 2: $\left\langle C, \eta_{0}\right\rangle=0$. De fato, como $C$ é uma corrente folheada, então é suficiente mostrar que $\left\langle C_{X(x)}, \eta_{0}\right\rangle=0$, mas isto segue da afirmação 1 , pois $\left\langle C_{X(x)}, \eta_{0}\right\rangle=\eta_{0}(X(x))$. Logo da afirmação 2 e de (3.17) segue que

$$
\langle C, \eta\rangle=\left\langle C_{X, \nu_{C}}, \eta\right\rangle, \forall \eta \in \mathcal{D}_{p}
$$

Sabemos que dado uma medida limitada não negativa $\nu$ em $M$, ela induz uma corrente de integração o qual é uma corrente folheada, pois $\nu$ pode-se aproximar arbitrariamente por combinações lineares finitas com coeficientes positivos de medidas de Dirac. 


\subsection{As medidas transversas invariantes são ciclos folhe- ados}

Nesta seção mostraremos que a cada ciclo folheado podemos associar uma medida transversa invariante por holonomia e vice-versa.

Proposição 3.29: A corrente $C_{\mu}$ associada a uma medida $\mu$ transversa invariante por holonomia definida no exemplo 3.15 é um ciclo folheado; i.e., $C_{\mu} \in \mathcal{Z}_{\mathcal{F}}$.

Demonstração. Temos que mostrar que $C_{\mu} \in \mathcal{Z}_{p} \cap \mathcal{C}_{\mathcal{F}}$. Afirmação 1: $C_{\mu} \in \mathcal{Z}_{p}$. De fato, provamos na seção 1 do capitulo 2 , que se $\omega$ for uma $p$-forma exata, então

$$
\left\langle C_{\mu}, \omega\right\rangle=0
$$

Por outro lado, seja $\eta \in \mathcal{D}_{p-1}$ qualquer, segue de (3.18)

$$
\left\langle\partial C_{\mu}, \eta\right\rangle=\left\langle C_{\mu}, d \eta\right\rangle=0
$$

o que prova a afirmação. Afirmação 2: $C_{\mu} \in \mathcal{C}_{\mathcal{F}}$. De fato, pela Proposição 3.28 é suficiente mostrar que $C_{\mu}$ é uma corrente de integração. Seja $\omega \in \mathcal{D}_{p}$, suponhamos que $\operatorname{supp}(\omega) \subset U_{\alpha}$, para algum $\alpha$. De (2.2) temos

$$
\left\langle C_{\mu}, \omega\right\rangle=\int_{\Sigma_{\alpha}}\left(\int_{\pi_{\alpha}^{-1}(y)}\right) d \mu(y)
$$

Definamos uma medida $\lambda_{y}$ em cada placa $\pi_{\alpha}^{-1}(y)$ por

$$
\lambda_{y}(A)=m\left(\varphi_{\alpha}(A)\right)
$$

onde $m$ é a medida de Lebesgue, se $f$ é integrável em $\pi_{\alpha}^{-1}(y)$ com respeito a medida $\lambda_{y}$, então

$$
\int_{\pi_{\alpha}^{-1}(y)} f(x) d \lambda_{y}(x)=\int_{\varphi_{\alpha}\left(\pi_{\alpha}^{-1}(y)\right)} f \circ \varphi_{\alpha} d m
$$

Definimos o funcional linear positivo

$$
\begin{aligned}
\Phi: C^{0}\left(U_{\alpha}\right) & \longrightarrow \mathbb{R} \\
h & \mapsto \int_{\Sigma_{\alpha}}\left(\int_{\pi_{\alpha}^{-1}(y)} h_{y}(x) d \lambda_{y}(x)\right) d \mu(y)
\end{aligned}
$$

Logo, pelo Teorema de representação de Riesz, existe uma única medida $\nu_{\alpha}$ em $U_{\alpha}$ tal que

$$
\Phi(h)=\int_{\Sigma_{\alpha}}\left(\int_{\pi_{\alpha}^{-1}(y)} h_{y}(x) d \lambda_{y}(x)\right) d \mu(y)=\int_{U_{\alpha}} h d \nu_{\alpha}, \forall h \in C^{0}\left(U_{\alpha}\right)
$$


Em particular, se $h=\omega(X) \operatorname{com} X$ um campo de $p$-vetores continuo, temos

$$
\int_{U_{\alpha}} \omega(X) d \nu_{\alpha}=\int_{\Sigma_{\alpha}}\left(\int_{\pi_{\alpha}^{-1}(y)} \omega(X)_{y}(x) d \lambda_{y}(x)\right) d \mu(y)
$$

Por outro lado, como $\omega=\sum_{|I|=p} \omega_{I} d x_{I}$ em $U_{\alpha}$ e $\left.\omega\right|_{\pi_{\alpha}^{-1}(y)}=\omega_{I_{0}} d x_{I_{0}}$, então

$$
\int_{\pi_{\alpha}^{-1}(y)} \omega=\int_{\pi_{\alpha}^{-1}(y)} \omega_{I_{0}} d x_{I_{0}}=\int_{\varphi_{\alpha}\left(\pi_{\alpha}^{-1}(y)\right)} \omega_{I_{0}} \circ \varphi_{\alpha}^{-1} d m
$$

Como $\left.\omega\right|_{\pi_{\alpha}^{-1}(y)}=\omega_{I_{0}} d x_{I_{0}} \Rightarrow \omega(X)_{y}=\omega_{I_{0}}$, então de (3.20) segue

$$
\int_{\pi_{\alpha}^{-1}(y)} \omega(X)_{y}(x) d \lambda_{y}(x)=\int_{\varphi_{\alpha}\left(\pi_{\alpha}^{-1}(y)\right)} \omega_{I_{0}} \circ \varphi_{\alpha}^{-1} d m
$$

Logo, de (3.22), (3.24) e (3.21) temos que

$$
\int_{U_{\alpha}} \omega(X) d \nu_{\alpha}=\int_{\Sigma_{\alpha}}\left(\int_{\pi_{\alpha}^{-1}(y)} \omega\right) d \mu(y)
$$

Pela forma como foi construído $\nu_{\alpha}$, temos que se $U_{\alpha} \cap U_{\beta} \neq \emptyset$ então $\nu_{\alpha}=\nu_{\beta}$ em $U_{\alpha} \cap U_{\beta}$. Assim podemos definir a seguente medida em $M$, como a suma disjunta destas medidas; i.e.,

$$
\nu:=\coprod_{\alpha} \nu_{\alpha}
$$

Logo, se consideramos $\left\{\delta_{\alpha}\right\}_{\alpha}$ partição da unidade subordinada a $\left\{U_{\alpha}\right\}_{\alpha}$. Seja $\eta \in \mathcal{D}_{p}$ arbitrário, então

$$
\begin{aligned}
\int_{M} \eta(X) d \nu & =\int_{M} \sum_{\alpha}\left(\delta_{\alpha} \eta\right)(X) d \nu \\
& =\sum_{\alpha} \int_{M}\left(\delta_{\alpha} \eta\right)(X) d \nu \\
& =\sum_{\alpha} \int_{U_{\alpha}}\left(\delta_{\alpha} \eta\right)(X) d \nu_{\alpha}
\end{aligned}
$$

Como $\operatorname{supp}\left(\delta_{\alpha} \eta\right) \subset U_{\alpha}$ segue de (3.24) que

$$
\int_{M} \eta(X) d \nu=\sum_{\alpha} \int_{\Sigma_{\alpha}}\left(\int_{\pi_{\alpha}^{-1}(y)} \delta_{\alpha} \eta\right) d \mu(y)
$$

Assim hemos provado que $\left\langle C_{X, \nu}, \eta\right\rangle=\left\langle C_{\mu}, \eta\right\rangle$; i.e., $C_{\mu}$ é uma corrente de integração.

Teorema 3.30 (D. Sullivan, [23]): Seja $\mathcal{F}$ uma folheação de dimensão $p$, transversalmente orientável e de classe $C^{1}$ de uma n-variedade compacta $M$ de classe $C^{\infty}$. Então as medidas transversas invariantes por holonomia e os ciclos folheados estão em correspondência canônica um a um. 
Observação 3.31: A correspondência canônica querê dizer o procedimento de associar uma corrente a uma medida transversa invariante como foi descrito no exemplo 3.15; isto é,

$$
<C_{\mu}, \omega>:=\sum_{\alpha \in I} \int_{\Sigma_{\alpha}}\left(\int_{\pi_{\alpha}^{-1}(x)} \lambda_{\alpha} \omega\right) d \mu(x)
$$

onde $\left\{\lambda_{\alpha}\right\}_{\alpha \in I}$ é uma partição da unidade associada à cobertura regular folheada $\left\{U_{\alpha}\right\}_{\alpha \in I}$, $\Sigma_{\alpha}$ é o espaço transverso em cada $U_{\alpha}$ e $\pi_{\alpha}: U_{\alpha} \rightarrow \Sigma_{\alpha}$ é a projeção canônica.

A Proposição 3.29 mostra que $C_{\mu}$ é um ciclo folheado, então unicamente resta demostrar o recíproco. Mas antes de começar a demostração é necessário mostrar alguns resultados prévios.

Consideremos um ciclo folheado $C$ não nulo de $\mathcal{F}$ e um campo continuo de $p$-vetores tangente a $\mathcal{F}$, que denotaremos por $X$. Suponhamos que para cada carta folheada $\varphi$ : $U \rightarrow \mathbb{R}^{p} \times \mathbb{R}^{q}$, regular de $\mathcal{F}$, o compacto $B=\varphi^{-1}\left(\mathbb{D}^{p} \times \mathbb{D}^{n-p}\right)$ satisfaz que $B \cap \operatorname{supp}(C) \neq \emptyset$, onde $\mathbb{D}^{p}$ e $\mathbb{D}^{n-p}$ são os discos fechados de raio um em $\mathbb{R}^{p}$ e $\mathbb{R}^{q}$ respectivamente.

Pela Proposição 3.28, existe uma única medida limitada e não negativa $\nu$ sobre $M$ tal que

$$
<C, \omega>=\int_{M} \omega(X) d \nu, \forall \omega \in \mathcal{D}_{p}
$$

Denotemos por $\bar{\nu}:=\left.\nu\right|_{B}$ a restrição de $\nu$ a $B$ e por $\tilde{\pi}$ e $\pi$ às projeções de $B$ sobre $P:=\mathbb{D}^{p}$, que identificaremos com as placas de $\mathcal{F}$ e $\Sigma=\mathbb{D}^{n-p}$ que identificaremos com o espaço transverso de $\mathcal{F}$ em $B$, respectivamente. Agora definamos $\tilde{\lambda}$ e $\lambda$ as medidas imagens direta de $\tilde{\pi}$ e $\pi$ respectivamente; i.e.,

$$
\tilde{\lambda}\left(A_{1}\right):=\bar{\nu}\left(\tilde{\pi}^{-1}\left(A_{1}\right)\right) \text { e } \lambda\left(A_{2}\right):=\bar{\nu}\left(\pi^{-1}\left(A_{2}\right)\right)
$$

onde $A_{1} \subset P$ e $A_{2} \subset \Sigma$ são subconjuntos de Borel.

Proposição 3.32: Podemos desintegrar a medida $\bar{\nu}$ e encontrar uma família $\left(\nu_{y}\right)_{y \in \Sigma}$ de medidas de probabilidade sobre $B$ tais que para todo $y \in \Sigma$ e toda função continua $f$ sobre $B$ tem-se:

1) $\operatorname{supp}\left(\nu_{y}\right) \subset \pi^{-1}(y)$.

2) A função $\int_{\pi^{-1}(y)} f d \nu_{y}$ sobre $\Sigma$ é $\lambda$-mensurável.

3) $\int_{B} f d \bar{\nu}=\int_{\Sigma}\left(\int_{\pi^{-1}(y)} f d \nu_{y}\right) d \lambda$ 
Demonstração. Seja $Z \subset B$ um boreliano para o qual $\tilde{\lambda} \times \lambda(A)=0 \operatorname{com} A=\tilde{\pi} \times \pi(Z)$ onde $\tilde{\lambda} \times \lambda$ é a medida produto, desde que

$$
\begin{aligned}
0=(\tilde{\lambda} \times \lambda) A & =(\tilde{\lambda} \times \lambda)(\tilde{\pi}(Z) \times \pi(Z)) \\
& =\tilde{\lambda}(\tilde{\pi}(Z)) \times \lambda(\pi(Z)) \\
& =\bar{\nu}\left(\tilde{\pi}^{-1}(\tilde{\pi}(Z))\right) \cdot \bar{\nu}\left(\pi^{-1}(\pi(Z))\right)
\end{aligned}
$$

e $Z \subset \tilde{\pi}^{-1}(\tilde{\pi}(Z)), \pi^{-1}(\pi(Z))$ temos que $\bar{\nu}(Z)=0$. Então, $\bar{\nu}<<\tilde{\lambda} \times \lambda$; i.e., $\bar{\nu}$ é absolutamente contínua com respeito à medida $\tilde{\lambda} \times \lambda$, segue pelo Teorema de Radom-Nikodym que existe $h \in L^{1}(\bar{\nu})$ tal que

$$
\int_{B} g d \bar{\nu}=\int_{B} g h d(\tilde{\lambda} \times \lambda), \forall g \bar{\nu} \text {-integrável. }
$$

Logo pelo Teorema de Fubini e como $P$ é identificado com as placas, tomando $P=\pi^{-1}(y)$ temos

$$
\int_{B} g d \bar{\nu}=\int_{B} g h d(\tilde{\lambda} \times \lambda)=\int_{\Sigma}\left(\int_{\pi^{-1}(y)}(g h)_{y}(x) d \tilde{\lambda}(x)\right) d \lambda(y)
$$

Tomando $\nu_{y}=h_{y} \tilde{\lambda} \Rightarrow \int_{\pi^{-1}(y)} g_{y} d \nu_{y}=\int_{\pi^{-1}(y)}(g h)_{y}(x) d \tilde{\lambda}(x)$. Assim

$$
\int_{B} g d \bar{\nu}=\int_{\Sigma}\left(\int_{\pi^{-1}(y)} g_{y} d \nu_{y}\right) d \lambda(y)
$$

Em particular temos (3.25) para $f$ contínua em $B$.

Observação 3.33: $\operatorname{Se} \omega \in \mathcal{D}_{p}, \operatorname{com} \operatorname{supp}(\omega) \subset \operatorname{int}(B)$, temos de (3.25)

$$
\langle C, \omega\rangle=\int_{M} \omega(X) d \nu=\int_{B} \omega(X) d \bar{\nu}=\int_{\Sigma}\left(\int_{\pi^{-1}(y)} \omega(X) d \nu_{y}\right) d \lambda(y) .
$$

Para $y \in T$, denotamos por $P_{y}$ a corrente integração definida por

$$
\left\langle P_{y}, \omega\right\rangle=\int_{\pi^{-1}(y)} \omega(X) d \nu_{y}
$$

Teorema 3.34: Existe uma única medida não negativa $\mu$ sobre o espaço transverso $\Sigma \grave{a}$ folheação $\mathcal{F}$ em $B$, tal que no interior de $B$ a corrente $C$ pode ser representado por

$$
\int_{\Sigma} C_{y} d \mu
$$

onde $C_{y}$ é a corrente folheada $\left\langle C_{y}, \eta\right\rangle=\int_{\pi^{-1}(y)} \eta, \forall \eta \operatorname{com} \sup (\eta) \subset \operatorname{int}(B)$ com a orientação de $\mathcal{F}$. 
Demonstração. Afirmação 1: $P_{y} \in \mathcal{Z}_{p}$ para $\lambda$-q.t.p $y \in \operatorname{int}(\Sigma)$; i.e., $\left\langle\partial P_{y}, \alpha\right\rangle=0$ para $\lambda$-q.t.p $y \in \operatorname{int}(\Sigma)$. De fato, como $C \in \mathcal{Z}_{\mathcal{F}},\langle C, d \alpha\rangle=\langle\partial C, \alpha\rangle=0$, para todo $\alpha \in \mathcal{D}_{p-1}$, em particular para $\alpha \in \mathcal{D}_{p-1}(\operatorname{int}(B))$, da Observação 3.33 segue que

$$
\int_{\Sigma}\left(\int_{\pi^{-1}(y)}(d \alpha)(X) d \nu_{y}\right) d \lambda=0
$$

Por outro lado, se $g$ é uma função de classe $C^{1}$ com suporte compacto contido em $\Sigma$, podemos aproximar $(g \circ \pi) \alpha$ por formas diferenciáveis de classe $C^{\infty}$ com suporte compacto contido em $\operatorname{int}(B)$, então de (3.27) temos

$$
\int_{\Sigma}\left(\int_{\pi^{-1}(y)}(d((g \circ \pi) \alpha))(X) d \nu_{y}\right) d \lambda=0
$$

$\log$, como $\left.(g \circ \pi)\right|_{\pi^{-1}(y)}$ é constante, temos $\left.(d(g \circ \pi) \wedge \alpha)\right|_{\pi^{-1}(y)} \equiv 0$. Assim,

$$
0=\int_{\Sigma}\left(\int_{\pi^{-1}(y)}(d((g \circ \pi) \alpha))(X) d \nu_{y}\right) d \lambda=\int_{\Sigma} g(y)\left(\int_{\pi^{-1}(y)}(d \alpha)(X) d \nu_{y}\right) d \lambda
$$

como $g$ é arbitrário, então $\int_{\pi^{-1}(y)}(d \alpha)(X) d \nu_{y}=0$ para $\lambda$-q.t.p $y \in \operatorname{int}(T)$. De $(3.26)$ temos

$$
\left\langle\partial P_{y}, \alpha\right\rangle=\left\langle P_{y}, d \alpha\right\rangle=0 \text { para } \lambda \text {-q.t.p } y \in \operatorname{int}(\Sigma)
$$

Em particular segue da afirmação que $\operatorname{supp}\left(\partial P_{y}\right) \cap \operatorname{int}(B)=\emptyset$ para $\lambda$-q.t.p $y \in \Sigma$.

Por outro lado, do Exemplo 3.23 temos que $Z_{p}=\left\langle C_{y}\right\rangle$. Logo, da afirmação 1, para cada $y \in \Sigma$ existe $k(y) \in \mathbb{R}$ tal que $P_{y}=k(y) C_{y}$, assim $k: \Sigma \rightarrow \mathbb{R}$ define uma função $\lambda$-mensurável. Tomando a medida $\mu=k \lambda$ em $\operatorname{int}(\Sigma)$. Para $\omega \in \mathcal{D}_{p}(\operatorname{int}(B))$ temos

$$
\begin{aligned}
\langle C, \omega\rangle & =\int_{\Sigma}(\underbrace{\left.\int_{\Sigma} \omega(X) d \nu_{y}\right)}_{\pi^{-1}(y)} d \lambda(y) \\
& =\int_{\left.T_{y}, \omega\right\rangle d \lambda}\left\langle P_{y}\right. \\
& =\int_{\Sigma}\left\langle k(y) C_{y}, \omega\right\rangle d \lambda \\
& =\int_{\Sigma} k_{y}\left\langle C_{y}, \omega\right\rangle d \lambda \\
& =\int_{\Sigma}\left\langle C_{y}, \omega\right\rangle d \mu
\end{aligned}
$$

Afirmação 2: $\mu$ é finita em compactos. De fato, seja $\eta$ uma $p$-forma em int $(B) \operatorname{com}$ suporte compacto na $x$-coordenada mas não na $y$-coordenada tal que $\int_{\pi^{-1}(y)} \eta(X) d \nu_{y}=1$, para 
cada $y \in T$. Se $h: \Sigma \rightarrow \mathbb{R}$ é de classe $C^{\infty}$ com suporte compacto. $h \circ \pi$ é uma função em $\operatorname{int}(B)$ tal que $\left.(h \circ \pi)\right|_{\pi^{-1}(y)}=$ cte, $\operatorname{logo}(h \circ \pi) \eta \in \mathcal{D}_{p}(\operatorname{int}(B))$ e da Observação 3.33 temos

$$
\begin{aligned}
\infty>\langle C,(h \circ \pi) \eta\rangle & =\int_{\Sigma}\left(\int_{\pi^{-1}(y)}((h \circ \pi) \eta)(X) d \nu_{y}\right) d \mu \\
& =\int_{\Sigma} h(\underbrace{\left.\int_{\pi^{-1}(y)} \eta(X) d \nu_{y}\right)}_{1} d \mu \\
& =\int_{\Sigma} h d \mu
\end{aligned}
$$

como $h$ foi arbitrário, segue que $\mu$ é finita em compactos.

Afirmação 3: $\mu$ é única. De fato, suponhamos que existe outra medida $\mu^{\prime}$ que satisfaz com as hipóteses do teorema. Pela afirmação $2, \mu$ e $\mu^{\prime}$ são finitas em compactos, assim podemos considerar a medida $\mu-\mu^{\prime}$, então

$$
\int_{\Sigma}\left(\int_{\pi^{-1}} \eta\right) d\left(\mu-\mu^{\prime}\right)=0, \forall \eta \in \mathcal{D}_{p}(\operatorname{int}(B))
$$

pelos mesmos argumentos da afirmação 2 , temos que

$$
0=\int_{\Sigma} h d\left(\mu-\mu^{\prime}\right), h \in C^{\infty} \text { com suporte compacto em } \Sigma
$$

assim, $\mu-\mu^{\prime}=0$ em conjuntos compactos. Por tanto $\mu=\mu^{\prime}$.

Demostração do Teorema 3.30. O teorema anterior nos proporciona a maneira de construir uma medida transversa a partir de um ciclo folheado em um compacto $B$ contido em uma carta regular folheada. Seja $\left\{B_{1}, \cdots, B_{m}\right\}$ uma cobertura de $M$, com $B_{\alpha}$ compacto como acima, tais que $\mathcal{U}=\left\{\operatorname{int}\left(B_{\alpha}\right)\right\}_{\alpha=1}^{m}$ é uma cobertura aberta de $M$ e denotamos por $\Sigma_{\alpha}$ o espaço transverso em cada $B_{\alpha}$. Pela proposição anterior podemos expressar $C$ em cada $B_{\alpha}$ como

$$
\int_{\Sigma_{\alpha}} C_{y} d \mu_{i}
$$

onde $C_{y}$ é a corrente de integração em $\pi^{-1}(y)$ e $\mu_{i}$ é a única medida dado pelo teorema anterior que permite expressar (3.28). Da teoria das folheações podemos supor(ver [7]) que se $\operatorname{int}\left(B_{\alpha}\right) \cap \operatorname{int}\left(B_{\beta}\right) \neq \emptyset$, existe uma carta regular folheada $W$ tal que $B_{\alpha} \cup B_{\beta} \subset W$. Pela unicidade das $\mu_{\alpha}^{\prime}$ s elas coincidem $\operatorname{int}\left(B_{\alpha}\right) \cap \operatorname{int}\left(B_{\beta}\right)$, assim são invariantes pelos difeomorfismos locais $\gamma_{\alpha}: \Sigma_{\alpha} \rightarrow \Sigma_{\beta}$ que geram o pseudogrupo de holonomia $\Gamma_{\mathcal{U}}$. Por tanto, podemos definir a medida em $\Sigma=\bigsqcup_{\alpha} \Sigma_{\alpha}$ de maneira natural como

$$
\mu=\coprod_{\alpha} \mu_{\alpha}
$$


Se $\left\{\lambda_{\alpha}\right\}$ é uma partição da unidade subordinada a $\mathcal{U}$, para cada $\omega \in \mathcal{D}_{p}$, temos que $\lambda_{\alpha} \omega \in \mathcal{D}_{p}\left(\operatorname{int}\left(B_{\alpha}\right)\right)$ e $\omega=\sum_{\alpha} \lambda_{\alpha} \omega$. Logo

$$
\begin{aligned}
\langle C, \omega\rangle & =\left\langle C, \sum_{\alpha} \lambda_{\alpha} \omega\right\rangle \\
& =\sum_{\alpha}\left\langle C, \lambda_{\alpha} \omega\right\rangle \\
& =\sum_{\alpha} \int_{\Sigma}\left\langle C_{y}, \lambda_{\alpha} \omega\right\rangle d \mu_{\alpha}(y) \\
& =\sum_{\alpha} \int_{\Sigma}\left(\int_{\pi_{\alpha}^{-1}(y)} \lambda_{\alpha} \omega\right) d \mu(y) \\
& =\left\langle C_{\mu}, \omega\right\rangle
\end{aligned}
$$

o que conclui a prova. 


\section{Capítulo}

\section{Exemplos Dinâmicos}

Neste capitulo estudaremos dois exemplos básicos, mas importantes da dinâmica hiperbólica.

\subsection{Thom-Arnold map}

Consideremos $A \in S L(n, \mathbb{Z})$ i.e, uma matriz com entradas inteiras e determinante \pm 1 , resulta que a matriz induz um difeomorfismo no toro $\mathbb{T}^{n}=\frac{\mathbb{R}^{n}}{\mathbb{Z}^{n}}$.

Definição 4.1: Seja $A \in S L(n, \mathbb{Z})$ hiperbólica. O difeomorfismo induzido $f: \mathbb{T}^{n} \rightarrow \mathbb{T}^{n}$ definida por

$$
f \circ \pi=\pi \circ A
$$

onde $\pi: \mathbb{R}^{n} \rightarrow \mathbb{T}^{n}$ é a projeção canônica é chamado difeomorfismo de Anosov linear.

Vamos a estudar sua riqueza dinâmica.

Teorema 4.2: Seja $f: \mathbb{T}^{n} \rightarrow \mathbb{T}^{n}$ um difeomorfismo de Anosov linear, então

1. $\overline{\operatorname{Per}(f)}=\mathbb{T}^{n}$

2. $f$ é transitivo e topologicamente mixing.

3. $f$ é expansivo.

4. Para qualquer $z \in \mathbb{T}^{n}$, as variedades $W^{s}(z)$ e $W^{u}(z)$ são densas em $\mathbb{T}^{n}$.

Demonstração: A prova sera feita para $A=\left(\begin{array}{ll}2 & 1 \\ 1 & 1\end{array}\right)$ neste caso $f$ é chamado ThomArnold map 
1) Seja $q \in \mathbb{Z}$ e consideremos o conjunto $C_{q}=\left\{\left(\frac{m}{q}, \frac{n}{q}\right) ; m, n \in \mathbb{Z}\right\}$, então $A\left(C_{q}\right)=C_{q}$ e como $f \circ \pi=\pi \circ A$ temos $f\left(\pi\left(C_{q}\right)\right)=\pi\left(C_{q}\right)$. Além disso $\pi\left(C_{q}\right)$ é um conjunto finito e como $f$ é injetiva temos que cada ponto de $\pi\left(C_{q}\right)$ é um ponto periódico. Por outro lado $\bigcup_{q \in \mathbb{Z}} C_{q}$ é denso em $\mathbb{R}^{2}$, assim $\bigcup_{q \in \mathbb{Z}} \pi\left(C_{q}\right)$ é denso em $\mathbb{T}^{2}$, pois tomemos $U$ aberto em $\mathbb{T}^{2}$ então $\pi^{-1}(U)$ é aberto em $\mathbb{R}^{2}$, logo

$$
\pi^{-1}(U) \cap \bigcup_{q \in \mathbb{Z}} C_{q} \neq \emptyset
$$

então

$$
U \cap \bigcup_{q \in \mathbb{Z}} \pi\left(C_{q}\right) \neq \emptyset
$$

e como $\bigcup_{q \in \mathbb{Z}} \pi\left(C_{q}\right) \subset \operatorname{Per}(f) \subset \mathbb{T}^{2}$ segue-se a afirmação.

2) Os autovalores de $A$ são $\lambda=\frac{3-\sqrt{5}}{2}$ e $\mu=\frac{3+\sqrt{5}}{2}$, assim

$$
E^{s}=\left\{\left(x,-\left(\frac{1+\sqrt{5}}{2}\right) x ; x \in \mathbb{R}\right\} \text { e } E^{u}=\left\{\left(x,\left(\frac{\sqrt{5}-1}{2}\right) x ; x \in \mathbb{R}\right\}\right.\right.
$$

Afirmação 1: $\pi\left(E^{s}\right)$ e $\pi\left(E^{u}\right)$ são densos em $\mathbb{T}^{2}$. De fato, como $\mathbb{T}^{2}=\frac{\mathbb{R}}{\mathbb{Z}} \times \frac{\mathbb{R}}{\mathbb{Z}}$ seja $p \in \mathbb{T}^{2}, p=\left(p_{1}, p_{2}\right)$ e considere o circulo

$$
S_{p}=\left\{\left(p_{1}, y\right): y \in \frac{\mathbb{R}}{\mathbb{Z}}\right\}
$$

por outro lado como $E^{s}=\left\{\left(x,-\left(\frac{1+\sqrt{5}}{2}\right) x ; x \in \mathbb{R}\right\}\right.$ então

$$
\pi\left(E^{s}\right)=\left\{\left(x,-\left(\frac{1+\sqrt{5}}{2}\right) x \bmod \mathbb{Z}^{2}, x \in \mathbb{R}\right\}\right.
$$

seja $k=-\frac{1}{2}(1+\sqrt{5}), \operatorname{logo}\left(p_{1}, k p_{1}\right) \in S_{p} \cap \pi\left(E^{s}\right)$. Consideremos a aplicação $T_{(1, k)}: \mathbb{T}^{2} \rightarrow \mathbb{T}^{2}$ dada por

$$
T_{(1, k)}(x, y)=(x+1, y+k) \bmod \mathbb{Z}^{2}
$$

como

$$
\mathcal{O}_{+}\left(p_{1}, k p_{1}\right)=\left\{T_{(1, k)}^{i}\left(p_{1}, k p_{1}\right) ; i \geq 0\right\}
$$

note que $T_{(1, k)}\left(p_{1}, k p_{1}\right)=\left(p_{1}+1, k p_{1}+k\right) \bmod \mathbb{Z}^{2}=\left(p_{1}+1, k\left(p_{1}\right)\right)$

$$
\begin{aligned}
T_{(1, k)}\left(p_{1}, k p_{1}\right) & =\left(p_{1}+1, k p_{1}+k\right) \bmod \mathbb{Z}^{2} \\
& =\left(p_{1}+1, k\left(p_{1}+1\right)\right) \bmod \mathbb{Z}^{2} \in \pi\left(E^{s}\right) \\
& =\left(p_{1}, k\left(p_{1}+1\right)\right) \bmod \mathbb{Z}^{2} \in S_{p}
\end{aligned}
$$


Assim em geral temos que $T_{(1, k)}^{n}\left(p_{1}, k p_{1}\right) \in \pi\left(E^{s}\right) \cap S_{p}$, para $n \geq 0$ e segue-se

$$
\mathcal{O}_{+}\left(p_{1}, k p_{1}\right) \subset \pi\left(E^{s}\right) \cap S_{p}
$$

Por outro lado, consideremos $h: S_{p} \rightarrow S^{1}$ dado por $h\left(p_{1}, y\right)=y$. Assim $h$ é uma homeomorfismo e

$$
R_{k} \circ h=h \circ T_{(1, k)}
$$

onde $R_{k}$ é a rotação de angulo $k$. Como $k \in \mathbb{I}$, então toda orbita de $R_{k}$ é densa e como $T_{(1, k)}$ é topologicamente conjugado com $R_{k}$, segue-se que $\mathcal{O}_{+}\left(p_{1}, k p_{1}\right)$ é densa em $S_{p}$. Por outro lado seja $U$ um aberto arbitrário em $\mathbb{T}^{2}$, seja $p \in U$, então pelo anterior temos que $\mathcal{O}_{+}\left(p_{1}, k p_{1}\right) \cap\left(S_{p} \cap U\right) \neq \emptyset$ e de (4.1) temos $\pi\left(E^{s}\right) \cap U \neq \emptyset$, o que prova que $\pi\left(E^{s}\right)$ é denso em $\mathbb{T}^{2}$. De forma análoga se mostra a densidade de $\pi\left(E^{u}\right)$.

Afirmação 2: Sejam $U$ e $V$ abertos de $\mathbb{R}^{2}$ tal que $U \cap E^{u} \neq \emptyset$ e $V \cap E^{s} \neq \emptyset$ então existe $n_{0} \in \mathbb{Z}^{+}$tal que $U \cap A^{n}(V) \neq \emptyset$ para todo $n \geq n_{0}$. De fato, como $\mathbb{R}^{2}=E^{s} \oplus E^{u}$ e seja uma norma adaptada em $\mathbb{R}^{2}$. Como $V \cap E^{s} \neq \emptyset$ tomemos $\left(x_{0}, 0\right) \in V \cap E^{s}$ desde que $V$ é aberto existe $\epsilon>0$ tal que

$$
Q=\left(x_{0}-\epsilon, x_{0}+\epsilon\right) \times(-\epsilon, \epsilon) \subset V
$$

como $A$ é hiperbólico e $\operatorname{dim} E^{s}=\operatorname{dim} E^{u}=1$, então

$$
A^{n}(Q)=\left(A^{n} x_{0}-\epsilon_{n}, A^{n} x_{0}+\epsilon_{n}\right) \times\left(-\delta_{n}, \delta_{n}\right)
$$

onde existe $\lambda>1$ tal que $\delta_{n} \geq \lambda^{n} \epsilon$ e $\left|\epsilon_{n}\right| \leq \lambda^{-n} \epsilon$, e $\lim _{n \rightarrow \infty} A^{n} x_{0}=0$. Por outro lado, como $U \cap E^{u} \neq \emptyset$ existe $\left(0, y_{0}\right) \in U \cap E^{u}$ e $\beta>0$ tal que

$$
Q_{1}=(-\beta, \beta) \times\left(y_{0}-\beta, y_{0}+\beta\right)
$$

escolha $n_{1}$ tal que se $n \geq n_{1}$, então

$$
\left(A^{n} x_{0}-\epsilon_{n}, A^{n} x_{0}+\epsilon_{n}\right) \subset(-\beta, \beta)
$$

e $n_{2}$ tal que $n \geq n_{2}, \operatorname{logo}$

$$
\left(y_{0}-\beta, y_{0}+\beta\right) \subset\left(-\delta_{n}, \delta_{n}\right)
$$

tomando $n_{0}=\max \left\{n_{1}, n_{2}\right\}$, então se $n \geq n_{0}$ vale (4.2) e (4.3) isto é $A^{n}(Q) \cap Q_{1} \neq \emptyset$, de onde segue-se a afirmação. 
Agora utilizando as duas afirmações anteriores provaremos que $f$ é topologicamente mixing. Sejam $U, V$ abertos qualquer em $\mathbb{T}^{2}$. Logo pela afirmação 1 temos $\pi\left(E^{s}\right) \cap$ $U \neq \emptyset$ e $\pi\left(E^{u}\right) \cap V \neq \emptyset$. Sejam $\tilde{U}$ e $\tilde{V}$ componentes conexas de $\pi^{-1}(U)$ e $\pi^{-1}(V)$ respectivamente(sem perdida de generalidade podemos supor que $\pi^{-1}(U)=\tilde{U}$ e $\left.\pi^{-1}(V)=\tilde{V}\right)$ tais que $E^{s} \cap \tilde{U} \neq \emptyset$ e $E^{u} \cap \tilde{V} \neq \emptyset$, pela afirmação 2 , existe $n_{0} \geq 0$ tal que

$$
A^{n}(\tilde{U}) \cap \tilde{V} \neq \emptyset \text {, para todo } n \geq n_{0}
$$

e como $\pi\left(A^{n}(\tilde{U}) \cap \tilde{V}\right) \subset f^{n}(U) \cap V$, então $f^{n}(U) \cap V \neq \emptyset$.

3) É Suficiente provar que $\mathbb{T}^{2}$ é um conjunto hiperbólico, pois se $\mathbb{T}^{2}$ é hiperbólico segue-se do corolário 1.21 que é expansivo. Sabemos que

$$
D \pi_{x}: T_{x} \mathbb{R}^{2} \rightarrow T_{[x]} \mathbb{T}^{2}
$$

é um isomorfismo linear. Como $T_{x} \mathbb{R}^{2}=\mathbb{R}^{2}=E^{s} \oplus E^{u}$, definamos $E_{[x]}^{s}:=D \pi_{[x]}\left(E^{s}\right)$ e $E_{[x]}^{u}:=D \pi_{[x]}\left(E^{u}\right)$. Então

$$
T_{[x]} \mathbb{T}^{2}=E_{[x]}^{s} \oplus E_{[x]}^{u}
$$

Seja $v \in E_{[x]}^{s} \cap E_{[x]}^{u} \Rightarrow \exists u_{1} \in E^{s}$ e $\exists u_{2} \in E^{u}$ tal que

$$
v=D \pi_{[x]}\left(u_{1}\right)=D \pi_{[x]}\left(u_{2}\right)
$$

como $D \pi_{[x]}$ é um isomorfismo, segue que $u_{1}=u_{2}$. Como $E^{s} \cap E^{u}=\{0\}$ segue que $u_{1}=u_{2}=0$ e $v=[0]$, logo $E_{[x]}^{s} \cap E_{[x]}^{u}=\{0\}$. Reciprocamente, seja $v \in T_{[x]} \mathbb{T}^{2}$, então $\exists ! u=D \pi_{x}^{-1}(v) \in \mathbb{R}^{2}$. Desse modo

$$
u=u_{1}+u_{2} \operatorname{com} u_{1} \in E^{s} \text { e } u_{2} \in E^{u}
$$

Logo, $v=D \pi_{x}(u)=D \pi_{x}\left(u_{1}\right)+D \pi_{x}\left(u_{2}\right)$. Onde $D \pi_{x}\left(u_{1}\right) \in E_{[x]}^{s}$ e $D \pi_{x}\left(u_{2}\right) \in E_{[x]}^{u}$. As outras condições de conjunto hiperbólico segue imediatamente do fato que $D \pi_{x}$ é isomorfismo linear e que $A$ é uma matriz hiperbólica.

4) Para provar que $\overline{W^{s}(\pi(x))}=\mathbb{T}^{2}$ é suficiente observar que $W^{s}(\pi(x))=\pi\left(x+E^{s}\right)$ e como $\pi\left(E^{s}\right)$ é denso em $\mathbb{T}^{2}$, então $\pi\left(x+E^{s}\right)$ é denso em $\mathbb{T}^{2}$ para todo $x \in \mathbb{R}^{2}$. Afirmação: $W^{s}(\pi(x))=\pi\left(x+E^{s}\right)$ e $W^{u}(\pi(x))=\pi\left(x+E^{u}\right)$. De fato, se $y \in$ $x+E^{s} \Rightarrow y-x \in E^{s}$, então

$$
\lim _{n \rightarrow \infty}\left\|A^{n}(y-x)\right\|=0 \Longleftrightarrow \lim _{n \rightarrow \infty}\left\|A^{n}(y)-A^{n}(x)\right\|=0
$$


assim

$$
\lim _{n \rightarrow \infty} d\left(f^{n}(\pi(x)), f^{n}(\pi(y))\right)=0
$$

$\operatorname{logo} \pi\left(x+E^{s}\right) \subset W^{s}(\pi(x))$. A volta se prova semelhantemente.

\subsection{Ferradura de Smale}

\subsubsection{Dinâmica simbólica}

Consideremos $A=\{1,2, \cdots, m\}$ um conjunto finito e $\Sigma=A^{\mathbb{Z}}$ o conjunto de todas as sequencias bilaterais com entradas elementos de $A$, isto é

$$
\Sigma=\left\{\theta=\left(\cdots, \theta_{-2}, \theta_{-1}, \theta_{0}, \theta_{1}, \theta_{2}, \cdots\right): \theta_{i} \in A, \text { para } i \in \mathbb{Z}\right\}
$$

Agora definamos shift de Bernoulli ou também chamada deslocamento à esquerda $\Sigma \rightarrow \Sigma$, dado por

$$
\sigma(\theta)=\theta^{\prime} \text { e } \theta_{i}^{\prime}=\theta_{i+1}
$$

Consideremos em $A$ a topologia discreta o qual é gerado pela métrica

$$
d_{A}(a, b)= \begin{cases}0 & \text { se } a=b \\ 1 & \text { se } a \neq b\end{cases}
$$

e dotando a $\Sigma$ com a topologia produto, temos que $\Sigma$ é compacto. Definimos

$$
d\left(\theta, \theta^{\prime}\right)=\sum_{i \in \mathbb{Z}} \frac{d_{A}\left(\theta_{i}, \theta_{i}^{\prime}\right)}{2^{|i|}}
$$

obtemos uma métrica em $\Sigma$ compatível com a topologia produto. Assim temos que $\sigma$ é um homeomorfismo, pois

$$
\begin{aligned}
d\left(\sigma(\theta), \sigma\left(\theta^{\prime}\right)\right. & =\sum_{i \in \mathbb{Z}} \frac{d_{A}\left(\sigma(\theta)_{i}, \sigma\left(\theta^{\prime}\right)_{i}\right)}{2^{|i|}} \\
& =\sum_{i \in \mathbb{Z}} \frac{d_{A}\left(\theta_{i+1}, \theta_{i+1}^{\prime}\right)}{2^{|i|}} \leq \frac{1}{2} d_{A}\left(\theta, \theta^{\prime}\right)
\end{aligned}
$$

Teorema 4.3: Seja $\sigma: \Sigma \rightarrow \Sigma$ o shift de Bernoulli, então:

1. $\overline{\operatorname{Per}(\sigma)}=\Sigma$

2. $\sigma$ é topologicamente mixing.

3. $\sigma$ é expansivo. 
4. $W^{s}(\alpha)$ e $W^{u}(\alpha)$ são densos em $\Sigma$ para todo $\alpha \in \Sigma$

\section{Demonstração.}

1) Seja $U$ um aberto em $\Sigma$, tomemos $\theta \in U$ então existe $\epsilon>0$ e $p \in \mathbb{N}$ tal que $B_{\epsilon}(\theta) \subset U$ e $\sum_{i \in \mathbb{Z}, i>p} \frac{1}{2^{|i|}}<\epsilon$. Agora definamos

$$
\theta^{\prime}=(\cdots, \underbrace{\theta_{-p}, \cdots, \theta_{-1}, \theta_{0}, \theta_{1}, \cdots, \theta_{p}}, \underbrace{\theta_{-p}, \cdots, \theta_{-1}, \theta_{0}, \theta_{1}, \cdots, \theta_{p}}, \cdots)
$$

então $\theta^{\prime} \in \operatorname{Per}(\sigma)$ e tem período $2 p+1$. Assim

$$
d\left(\theta, \theta^{\prime}\right)=\sum_{i \in \mathbb{Z},|i|>p} \frac{d_{A}\left(\theta_{i}, \theta_{i}^{\prime}\right)}{2^{|i|}}<\sum_{i \in \mathbb{Z}, i>p} \frac{1}{2^{|i|}}<\epsilon
$$

e segue-se que $\theta^{\prime} \in B_{\epsilon}(r)$ o que prova que $U \cap \operatorname{Per}(\sigma) \neq \emptyset$.

2) Sejam $\alpha, \alpha^{\prime} \in \Sigma$ e os abertos básicos

$$
B_{N_{1}}=\left\{\theta \in \Sigma: \theta_{i}=\alpha_{i}, \forall|i| \leq N_{1}\right\}
$$

e

$$
B_{N_{2}}=\left\{\theta \in \Sigma: \theta_{i}=\alpha_{i}^{\prime}, \forall|i| \leq N_{2}\right\}
$$

tomemos $m>N_{1}+2 N_{2}+1$ fixo, então para todo $k \geq m$ definimos $\theta \in \Sigma$ tal que

$$
\theta_{i}=\alpha_{i}, \forall|i| \leq N_{1} \text { e } \theta_{i+k}=\alpha_{i}^{\prime}, \forall|i| \leq N_{2}
$$

e temos que $\theta \in B_{N_{1}}$ e $\sigma^{-k}(\theta) \in B_{N_{2}}$, o que significa

$$
B_{N_{1}} \cap \sigma^{k}\left(B_{N_{2}}\right) \neq \emptyset
$$

3) Seja $\delta<1$ e $\theta, \theta^{\prime} \in \Sigma$ tal que

$$
d\left(\sigma^{n}(\theta), \sigma^{n}\left(\theta^{\prime}\right)\right)<\delta, \forall n \in \mathbb{Z}
$$

Suponha que $\theta \neq \theta^{\prime}$, então existe $m>0$ tal que $\theta_{m} \neq \theta_{m}^{\prime}$, logo

$$
1 \leq d\left(\sigma^{-m}(\theta), \sigma^{-m}\left(\theta^{\prime}\right)\right)
$$

o que é uma contradição. 
4) É suficiente observar que

$$
W^{s}(\alpha)=\left\{\theta \in \Sigma: \theta_{i}=\alpha_{i}, \forall i \geq k, \text { para algum } k \in \mathbb{Z}\right\}
$$

$\mathrm{e}$

$$
W^{u}(\alpha)=\left\{\theta \in \Sigma: \theta_{i}=\alpha_{i}, \forall i \leq k, \text { para algum } k \in \mathbb{Z}\right\}
$$

pois, seja $B_{N}=\left\{\theta \in \Sigma: \theta_{i}=\alpha_{i}\right.$, para $\left.|i| \leq N\right\}$ o aberto básico para algum $N \in \mathbb{N}$, então $W^{s}(\alpha) \cap B_{N} \neq \emptyset$.

\subsubsection{Ferradura de Smale}

Consideremos um difeomorfismo $f: \mathbb{R}^{2} \rightarrow \mathbb{R}^{2}$ que age no quadrado unitário $Q$ como passamos a descrever. Primeiro contrai linearmente o quadrado na direção horizontal por um fator $\delta<\frac{1}{2}$ e expande-o na direção vertical por um fator $>2$ obtemos um retângulo que é dobrado na forma de $\bigcap$ ("ferradura") e colocado sobre o quadrado original $Q$ como mostra a figura 4.1 .
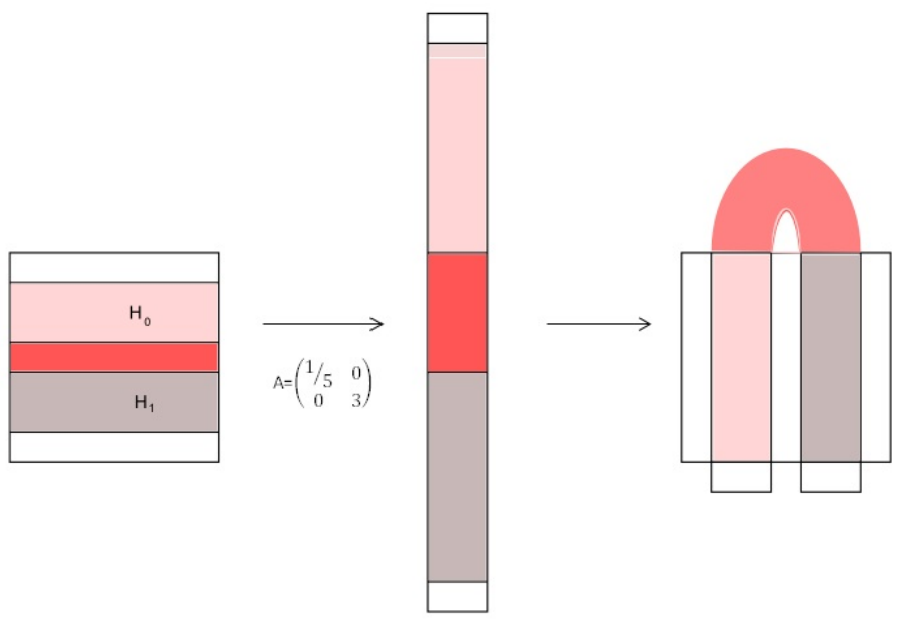

Figura 4.1: ferradura de Smale

Agora consideremos o seguente conjunto $\Lambda=\bigcap_{n \in \mathbb{Z}} f^{n}(Q)$. Nos estamos interessados em estudar a dinâmica de $f$ neste conjunto.

Proposição 4.4: $\Lambda$ é um conjunto hiperbólico.

Demonstração. Segue imediato que $\Lambda$ é compacto e invariante por $f$. Por outro lado segue-se o seguinte gráfico da forma como $f$ age em $Q$. 


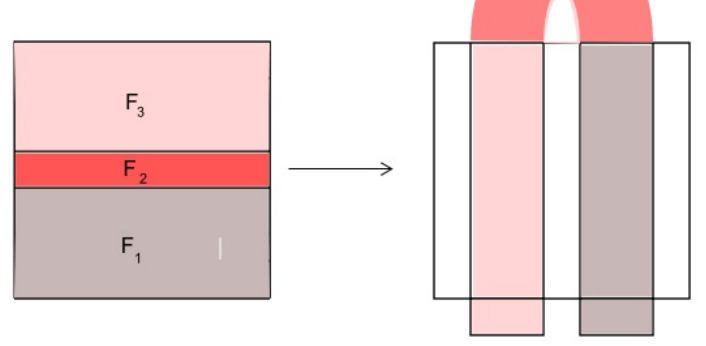

Figura 4.2:

Assim, temos o seguente:

. $\Lambda \cap F_{2}=\emptyset$.

- $\left.f\right|_{F_{1}}(x)=A x+c$, (é uma transformação linear e uma translação).

- $\left.f\right|_{F_{3}}(x)=-A x+c$, (é uma transformação linear,uma rotação e uma translação).

Então, para qualquer $x \in F_{1} \cup F_{3}$, escolha

$$
E^{s}(x)=\langle(1,0)\rangle \text { e } E^{u}(x)=\langle(0,1)\rangle
$$

de onde segue que $\mathbb{R}^{2}=E^{s}(x) \oplus E^{u}(x)$. Como $D\left(\left.f\right|_{F_{1}}\right)_{x}=A$ e $D\left(\left.f\right|_{F_{3}}\right)_{x}=-A$, então

$$
D\left(\left.f\right|_{F_{1}}\right)_{x}\left(E^{s}(x)\right)=E^{s}(f(x)) \text { e } D\left(\left.f\right|_{F_{1}}\right)_{x}\left(E^{u}(x)\right)=E^{u}(f(x))
$$

Se $v \in E^{s}(x)$ então $\left\|D\left(\left.f\right|_{F_{1}}\right)_{x} v\right\|=\frac{1}{5}\|v\|$ e se $v \in E^{u}(x)$ então $\left\|D\left(\left.f\right|_{F_{1}}\right)_{x}^{-1} v\right\|=\frac{1}{3}\|v\|$. O mesmo temos para $D\left(\left.f\right|_{F_{3}}\right)_{x}$ e $D\left(\left.f\right|_{F_{3}}\right)_{x}^{-1}$. Como $\Lambda \subset F_{1} \cup F_{3}$, segue-se que $\Lambda$ é um conjunto hiperbólico.

O teorema seguinte mostra que este conjunto é um conjunto totalmente desconexo.

Teorema 4.5: $\Lambda=K_{1} \times K_{2}$, onde $K_{1}$ e $K_{2}$ são conjuntos de cantor em $[0,1]$.

Demonstração. Pela forma como foi construído a função, temos que

- $Q \cap f(Q)=$ duas faixas verticais cada uma de largura $\frac{1}{5}$

- $f^{-1}(Q) \cap Q=$ duas faixas horizontais cada uma de altura $\frac{1}{3}$. 


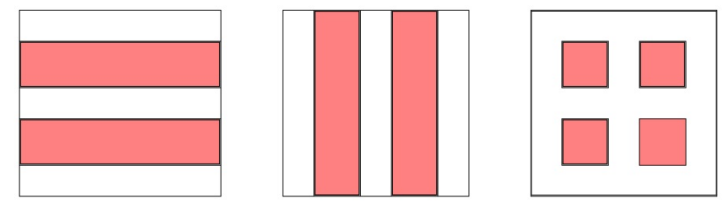

Figura 4.3: $f^{-1}(Q) \cap Q \cap f(Q)$

Como $Q \cap f(Q) \cap f^{2}(Q)=f\left(f(Q) \cap V_{0}\right) \cup f\left(f(Q) \cap V_{1}\right)$, então:

- $Q \cap f(Q) \cap f^{2}(Q)=4$ faixas verticais cada uma com largura $\frac{1}{5^{2}}$. E também temos

- $f^{-2}(Q) \cap f^{-1}(Q) \cap Q=4$ faixas horizontais cada uma de altura $\frac{1}{3^{2}}$.
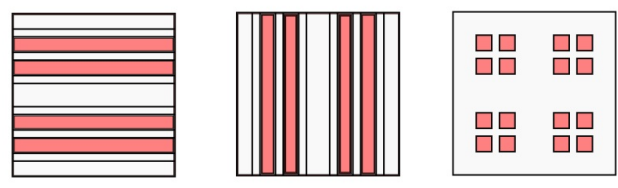

Figura 4.4: $\cap_{i=-2}^{2} f^{-i}(Q)$

Assim procedendo de forma indutiva se mostra que:

- $\bigcap_{i=0}^{n} f^{i}(Q)=2^{n}$ faixas verticais com largura $\frac{1}{5^{n}}$, e cada faixa vertical contém exatamente 2 faixas do próximo. Então temos que

$$
\bigcap_{i=0}^{\infty} f^{i}(Q)=K_{1} \times[0,1] \text {, onde } K_{1} \text { é um conjunto de Cantor em [0,1]. }
$$

De forma análoga segue-se

$\bigcap_{i=-n}^{0} f^{i}(Q)=2^{n}$ faixas horizontais com altura $\frac{1}{3^{n}}$, e cada faixa horizontal contém exatamente 2 faixas do próximo. Então temos que

$$
\bigcap_{i=\infty}^{0} f^{i}(Q)=[0,1] \times K_{2} \text {, onde } K_{2} \text { é um conjunto de Cantor em }[0,1] .
$$

De onde se conclui o teorema.

Definamos a seguente aplicação $h: \Lambda \rightarrow \Sigma_{2}$ dada por

$$
h(x)= \begin{cases}0 & \text { se } f^{i}(x) \in H_{0} \\ 1 & \text { se } f^{i}(x) \in H_{1}\end{cases}
$$

onde $\Sigma_{2}=\{0,1\}^{\mathbb{Z}}$ e $H_{0}, H_{1}$ são como na figura 4.1. 
Proposição 4.6: hé uma conjugação topológica entre $\left.f\right|_{\Lambda}$ e o shift $\sigma: \Sigma_{2} \rightarrow \Sigma_{2}$.

Demonstração. Afirmação 1: $\left.h \circ f\right|_{\Lambda}=\sigma \circ h$. De fato, consideremos um ponto $q \in \Lambda$, e ponhamos $\alpha=h(q)$ e $\theta=h(f(q))$. Deste modo, $f^{j+1}(q) \in H_{\alpha_{j+1}}$ e $f^{j+1}(q)=f^{j}(f(q)) \in$ $H_{\theta_{j}}$. Assim $\alpha_{j+1}=\theta_{j}$ e, por tanto, $\sigma(\alpha)=\theta$, isto é, $\sigma(h(q))=h(f(q))$.

Afirmação 2: $h$ é continua. De fato, seja $q \in \Lambda, \alpha=h(q)$. Seja $N$ fixo e consideremos

$$
B=\left\{\theta: \theta_{j}=\alpha_{j} \text { para }|j|<N\right\}
$$

uma vizinhança de $\alpha$. A continuidade $f$ garante que existe $\delta>0$ tal que para $p \in \Lambda$ com $\|p-q\|<\delta, f^{j}(p) \in H_{\alpha_{j}}$ para $j \in\{-N, \cdots, N\}$. Assim, se $\theta=h(p)$ e $\|p-q\|<\delta$ tem-se $\theta \in B$, o que prova a continuidade de $h$.

Afirmação 3: $h$ é sobrejetiva. De fato, Seja $\alpha \in \Sigma_{2}$, vamos construir um elemento $q \in \Lambda$ tal que $h(q)=\alpha$. Vamos mostrar por indução que $\bigcap_{j=1}^{n} f^{j}\left(H_{\alpha_{-j}}\right)$ é uma faixa vertical de largura $\frac{1}{5^{n}}$, para todo $\alpha \in \Sigma_{2}$.

Para $n=1, f\left(H_{\alpha_{-1}}\right)=V_{\alpha_{-1}}$ é uma faixa vertical de largura $\frac{1}{5}$. Vamos supor que é valido para $n-1$, como

$$
\bigcap_{j=1}^{n} f^{j}\left(H_{\alpha_{-j}}\right)=f\left(\bigcap_{j=2}^{n} f^{j}\left(H_{\alpha_{-j}}\right) \cap f\left(H_{\alpha_{-1}}\right)\right.
$$

Pela hipótese indutiva $\bigcap_{j=2}^{n} f^{j}\left(H_{\alpha_{-j}}\right)$ é uma faixa vertical de largura $\frac{1}{5^{n-1}}$ e $f\left(H_{\alpha_{-1}}\right)$ é uma faixa vertical de largura $\frac{1}{5}$, o que prova nossa afirmação. Assim, quando $n \rightarrow+\infty$, concluímos que $\bigcap_{j=1}^{\infty} f^{j}\left(H_{\alpha_{-j}}\right)$ é um segmento de reta vertical.

De forma análoga mostra-se que $\bigcap_{j=-\infty}^{0} f^{j}\left(H_{\alpha_{-j}}\right)$ é um segmento de reta horizontal. Assim, o conjunto

$$
\bigcap_{j=-\infty}^{\infty} f^{j}\left(H_{\alpha_{-j}}\right)
$$

é formado apenas por um ponto $q$.

Afirmação 4: $h$ é injetiva. De fato, suponhamos que $h(p)=h(q)=\alpha$. Para qualquer $j$, temos que $f^{-j}(p), f^{-j}(q) \in H_{\alpha_{-j}}$, pelo que $p, q \in f^{j}\left(H_{\alpha_{-j}}\right)$. Como $j$ é qualquer, resulta que $p, q \in \bigcap_{j=1}^{\infty} f^{j}\left(H_{\alpha_{-j}}\right)$,e, portanto, $p$ e $q$ estão no mesmo segmento de reta vertical; da mesma forma, $p, q$ estão na mesma reta horizontal. Assim, e porque o conjunto $\bigcap_{j=-\infty}^{\infty} f^{j}\left(H_{\alpha_{-j}}\right)$ é um conjunto singular, concluímos que $p=q$.

Afirmação 5: $h^{-1}$ é continua. De fato, isto segue imediatamente da afirmação 1 e do fato que $\Lambda$ e $\Sigma_{2}$ são compactos e Hausdorff.

$\left.f\right|_{\Lambda}$ é chamado ferradura de Smale. 
Teorema 4.7: Seja $\left.f\right|_{\Lambda}: \Lambda \rightarrow \Lambda$ a ferradura de Smale. Então
i) $\overline{\operatorname{Per}\left(\left.f\right|_{\Lambda}\right)}=\Lambda$.
ii) $\left.f\right|_{\Lambda}$ é topologicamente mixing.
iii) $\left.f\right|_{\Lambda}$ é expansivo.
iv) $W^{s}(x) \cap \Lambda$ e $W^{u}(x) \cap \Lambda$ são densos em $\Lambda$, para todo $x \in \Lambda$.

Demonstração. A prova segue-se imediatamente da Proposição 1.27 e Proposição 4.6. 


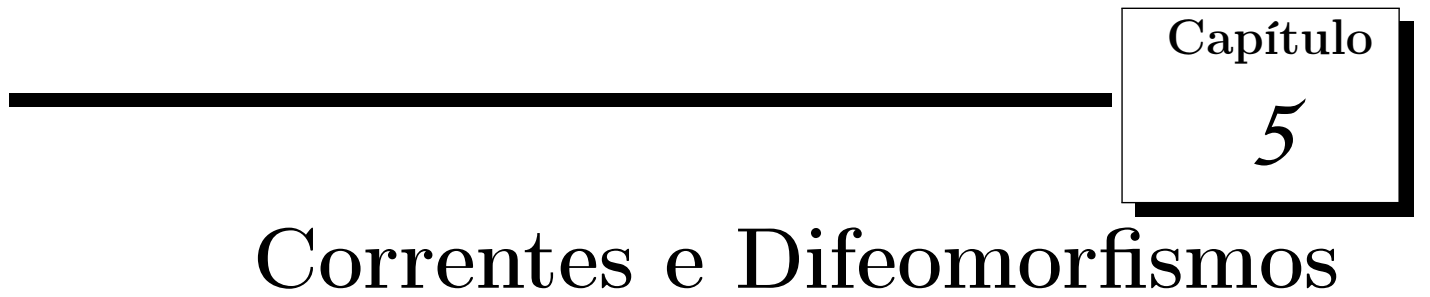

R. Bowen [4], [5] mostra que se $f: M \rightarrow M$ é um difeomorfismo topologicamente mixing, então existe uma medida de Bowen $\mu$ em M; isto é, a única medida que máxima a entropia métrica de $f$. Neste capítulo nós apresentamos a prova deste resultado, para o qual relacionamos o difeomorfismo hiperbólico com o shifts de tipo finito usando partições de Markov. Mas o resultado principal deste capítulo é a apresentação o trabalho de RuelleSullivan [19], eles mostram que a medida de Bowen pode ser expressado como o produto de duas medidas transversas invariantes para as folheações estáveis e instáveis(ver Teorema 5.8). Por último, mostramos que os difeomorfismos de Anosov topologicamente mixing, que preservam a orientação das folhas estáveis e folhas instáveis induzem elementos da cohomologia de DeRham(ver Teorema 5.9).

\subsection{Medida de máxima entropia}

Seja $f: M \rightarrow M$ um difeomorfismo $C^{r}$ e $\Lambda$ um conjunto hiperbólico com estrutura de produto local. Suponhamos que $\Lambda$ possui uma partição de Markov $\mathcal{R}=\left\{R_{1}, \cdots, R_{m}\right\}$.

Definição 5.1: A matriz de transição $A$ da partição de Markov $\mathcal{R}$ é uma matriz $m \times m$ definido como

$$
A_{i j}= \begin{cases}1 & \text { se } \operatorname{int} R_{i} \cap f^{-1}\left(\operatorname{int} R_{j}\right) \neq \emptyset \\ 0 & \text { se } \operatorname{int} R_{i} \cap f^{-1}\left(\operatorname{int} R_{j}\right)=\emptyset\end{cases}
$$

Assim podemos considerar $\Sigma_{A}=\left\{\theta \in \Sigma: A_{\theta_{i} \theta_{i+1}}=1\right.$ para todo $\left.i \in \mathbb{Z}\right\}$ o conjunto compacto e invariante pelo shift $\sigma$. Assim temos a seguinte proposição .

Proposição 5.2: Seja $\mathcal{R}=\left\{R_{1}, \cdots, R_{m}\right\}$ uma partição de Markov de $\Lambda$. Então para todo $\theta \in \Sigma_{A}$ o conjunto 


$$
\bigcap_{j \in \mathbb{Z}} f^{-j}\left(R_{\theta_{j}}\right)
$$

contém um único ponto de $\Lambda$ e a aplicação $\pi: \Sigma_{A} \rightarrow \Lambda$ definida como

$$
\pi(\theta)=\bigcap_{j \in \mathbb{Z}} f^{-j}\left(R_{\theta_{j}}\right)
$$

satisfaz:

i) $\pi$ é uma semi-conjugação de $\sigma$ e $\left.f\right|_{\Lambda}$; i.e.,

a) $\pi$ é contínua e sobrejetiva.

b) $f \circ \pi=\pi \circ \sigma$.

ii) $\pi$ é injetiva em $\pi^{-1}\left(\Lambda \backslash \bigcup_{j \in \mathbb{Z}} f^{-j} \partial \mathcal{R}\right)$.

iii) Para toda medida $\mu \in M_{\sigma}\left(\Sigma_{A}\right)$ ergódica e positiva sobre abertos, temos

$$
\mu\left(\pi^{-1}\left(\bigcup_{j \in \mathbb{Z}} f^{-j} \partial \mathcal{R}\right)\right)=0
$$

iv) Se $\left.f\right|_{\Lambda}$ é topologicamente mixing, então $\sigma$ é topologicamente mixing.

Antes de fazer a prova da Proposição, mostraremos o seguinte Lema.

Lema 5.3: Dado $N \in \mathbb{N}$, então o conjunto

$$
K_{N}(\theta)=\bigcap_{j=-N}^{N} f^{-j} R_{\theta_{j}}
$$

é um retângulo fechado, para todo $\theta \in \Sigma_{A}$.

Demonstração. Suponhamos no primeiro lugar que $K_{N}(\theta) \neq \emptyset$, então mostraremos que $K_{N}(\theta)$ é um retângulo. Seja $x, y \in K_{N}(\theta)$, então $f^{j}(x), f^{j}(y) \in R_{\theta_{j}}$ para $|j| \leq N$, assim $w=[x, y] \in R_{\theta_{0}}, w \in W^{s}\left(x, R_{\theta_{0}}\right)$ e $w \in W^{u}\left(y, R_{\theta_{0}}\right)$, segue-se da Proposição 1.37 que

$$
f(w) \in W^{s}\left(f(x), R_{\theta_{1}}\right) \text { e } f^{-1}(w) \in W^{u}\left(f^{-1}(y), R_{\theta_{-1}}\right)
$$

pois $x \in R_{\theta_{0}} \cap f^{-1}\left(R_{\theta_{1}}\right)$ e $f^{-1}(y) \in R_{\theta_{-1}} \cap f^{-1}\left(R_{\theta_{0}}\right)$. Assim, de forma geral temos que

$$
f^{j}(w) \in W^{s}\left(f^{j}(x), R_{\theta_{j}}\right) \text { e } f^{-j}(w) \in W^{u}\left(f^{-j}(y), R_{\theta_{-j}}\right), \text { para } 0 \leq j \leq N .
$$


Observe-se que para aplicar a Proposição 1.37 faz uso que $\theta \in \Sigma_{A}$. De onde segue que $f^{-j}(w) \in R_{\theta_{j}}$ para $|j| \leq N$. Por tanto $w \in K_{N}(\theta)$. Agora mostraremos que $K_{N}(\theta) \neq \emptyset$, como

$$
\begin{aligned}
K_{N}(\theta) & =f^{N} R_{\theta_{-N}} \cap f^{N-1} R_{\theta_{-N+1}} \cap \cdots \cap f^{-N+1} R_{\theta_{N-1}} \cap f^{-N} R_{\theta_{N}} \\
& =f^{N}\left(R_{\theta_{-N}} \cap f^{-1} R_{\theta_{-N+1}} \cap \cdots \cap f^{-2 N} R_{\theta_{N}}\right)
\end{aligned}
$$

então é suficiente demostrar que para todo $\alpha \in \Sigma_{A}$ e todo $n \geq 0$

$$
R_{\alpha_{0}} \cap f^{-1} R_{\alpha_{1}} \cap \cdots \cap f^{-n} R_{\alpha_{n}} \neq \emptyset
$$

isto provaremos por indução.

Para $n=0 \Rightarrow R_{\alpha_{0}} \neq \emptyset$. Suponhamos que $R_{\alpha_{1}} \cap f^{-1} R_{\alpha_{2}} \cap \cdots \cap f^{-n+1} R_{\alpha_{n}} \neq \emptyset$. Agora mostraremos que vale para $n$. Seja $y \in R_{\alpha_{1}} \cap f^{-1} R_{\alpha_{2}} \cap \cdots \cap f^{-n+1} R_{\alpha_{n}}$. Como

$$
\alpha \in \Sigma_{A} \Rightarrow \operatorname{int} R_{\alpha_{0}} \cap f^{-1} \operatorname{int} R_{\alpha_{1}} \neq \emptyset \Rightarrow \exists f^{-1}(x) \in R_{\alpha_{0}} \cap f^{-1} R_{\alpha_{1}}
$$

Seja $z=[y, x]$, então $z \in R_{\alpha_{1}}$ e $z \in W^{s}\left(y, R_{\alpha_{1}}\right)$. Desde que $y \in R_{\alpha_{1}} \cap f^{-1} R_{\alpha_{2}}$ segue da Proposição 1.37 que

$$
f W^{s}\left(y, R_{\alpha_{1}}\right) \subset W^{s}\left(f(y), R_{\alpha_{2}}\right) \Rightarrow f(z) \in W^{s}\left(f(y), R_{\alpha_{2}}\right)
$$

De forma análoga se prova que

$$
f^{2}(z) \in W^{s}\left(f^{2}(y), R_{\alpha_{3}}\right), \cdots, f^{n-1}(z) \in W^{s}\left(f^{n-1}(y), R_{\alpha_{n}}\right)
$$

assim,

$$
z \in R_{\alpha_{1}} \cap f^{-1} R_{\alpha_{2}} \cap \cdots \cap f^{-n+1} R_{\alpha_{n}}
$$

Por outro lado, desde que $f^{-1}(x) \in R_{\alpha_{0}} \cap f^{-1} R_{\alpha_{1}}$, segue da Proposição 1.37 que

$$
f W^{u}\left(f^{-1}, R_{\alpha_{0}}\right) \supset W^{u}\left(x, R_{\alpha_{1}}\right)
$$

então $f^{-1}(z) \in W^{u}\left(f^{-1}, R_{\alpha_{0}}\right)$ pois $z \in W^{u}\left(x, R_{\alpha_{1}}\right)$. Pelo tanto

$$
f^{-1}(z) \in R_{\alpha_{0}} \cap f^{-1} R_{\alpha_{1}} \cap \cdots \cap f^{-n} R_{\alpha_{n}}
$$

Demostração da Proposição 5.2. Para cada $N \in \mathbb{N}$ seja o conjunto $K_{N}(\theta)=\bigcap_{j=-N}^{N} f^{-j} R_{\theta_{j}}$, no lema anterior vimos que $K_{N}(\theta)$ é fechado não vazio em $\Lambda$. Como $\Lambda$ é compacto, então $K_{N}(\theta)$ é compacto. Por construção temos

$$
K_{N}(\theta) \supset K_{N+1}(\theta)
$$


então

$$
\bigcap_{j=-\infty}^{\infty} f^{-j} R_{\theta_{j}}=\bigcap_{N=1}^{\infty} K_{N}(\theta) \neq \emptyset
$$

o qual prova que existe $x \in \bigcap_{j \in \mathbb{Z}} f^{-j} R_{\theta_{j}}$ e é único, pois se $x, y \bigcap_{j \in \mathbb{Z}} f^{-j} R_{\theta_{j}}$ então $f^{j}(x), f^{j}(y) \in$ $R_{\theta_{j}} \forall j \in \mathbb{Z}$. Mas $R_{\theta_{j}}$ tem diâmetro menor que a constante de expansividade de $f$, resultando $x=y$. Agora provaremos os seguintes itens.

i) Provamos que $\pi$ é uma semi-conjugação de $\sigma$ e $\left.f\right|_{\Lambda}$.

$$
\pi(\sigma(\theta))=\bigcap_{j \in \mathbb{Z}} f^{-j} R_{\theta_{j+1}}=f\left(\bigcap_{j \in \mathbb{Z}} f^{-j-1} R_{\theta_{j+1}}\right)=f(\pi(\theta))
$$

então

$$
\pi \circ \sigma(\theta)=f \circ \pi(\theta) \forall \theta \in \Sigma_{A}
$$

pelo tanto é comutativo o seguinte diagrama

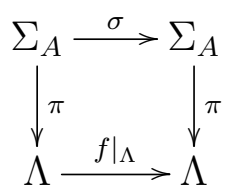

Afirmação: $\pi$ é continua. De fato, se $\theta^{n} \rightarrow \theta \in \Sigma_{A}$, chamando $\pi\left(\theta^{n}\right)=x_{n} \in \Lambda$ e como $\Lambda$ é compacto $\left(x_{n}\right)_{n}$ admite uma subsequencia convergente, sem perda de generalidade vamos supor que $x_{n} \rightarrow x \in \Lambda$ e além disso

$$
x_{n}=\bigcap_{j \in \mathbb{Z}} f^{-j} R_{\theta_{j}^{n}}
$$

Como $\theta^{n} \rightarrow \theta$, dado $p>0$ existe $N>0$ tal que $\theta_{j}^{n}=\theta_{j} \forall n>N, \forall|j| \leq p$. Logo para todo $n>N$ o ponto $x_{n}=\bigcap_{j=-p}^{p} f^{-j} R_{\theta_{j}}$. Como $\bigcap_{j=-p}^{p} f^{-j} R_{\theta_{j}}$ é fechado e $x_{n} \rightarrow x$. Então

$$
x \in \bigcap_{j=-p}^{p} f^{-j} R_{\theta_{j}} \forall p>0 \Rightarrow x \in \bigcap_{j \in \mathbb{Z}} f^{-j} R_{\theta_{j}}=\pi(\theta)
$$

Afirmação: $\pi$ é sobrejetiva, pois, se $x \in \Lambda \backslash \bigcup_{j \in \mathbb{Z}} f^{-j} \partial \mathcal{R}$ a orbita por $x$ não corta ao bordo $\partial \mathcal{R}$. Então para cada $j$, seja $\theta_{j}(x)$ o único subíndice tal que $f^{j}(x) \in \operatorname{int} R_{\theta_{j}(x)}$. Assim

$$
f^{j}(x) \in \operatorname{int} R_{\theta_{j}(x)} \cap f^{-1} \operatorname{int} R_{\theta_{j+1}(x)}
$$

$\log$, 


$$
A_{\theta_{j} \theta_{j+1}}=1 \Rightarrow \theta_{j}(x) \in \Sigma_{A} \Rightarrow x \in \bigcap_{j \in \mathbb{Z}} f^{-j} R_{\theta_{j}(x)}
$$

Assim temos provado que $\Lambda \backslash \bigcup_{j \in \mathbb{Z}} f^{-j} \partial \mathcal{R} \subset \pi\left(\Sigma_{A}\right)$. Por outro lado da Proposição 1.36 temos que $\Lambda \backslash \bigcup_{j \in \mathbb{Z}} f^{-j} \partial \mathcal{R}$ é denso, e desde que $\pi\left(\Sigma_{A}\right)$ é fechado pois $\Sigma_{A}$ é compacto, segue que

$$
\pi\left(\Sigma_{A}\right)=\overline{\pi\left(\Sigma_{A}\right)} \supset \overline{\Lambda \backslash \bigcup_{j \in \mathbb{Z}} f^{-j} \partial \mathcal{R}}=\Lambda .
$$

ii) Sejam $\theta, \theta^{\prime} \in \pi^{-1}\left(\Lambda \backslash \bigcup_{j \in \mathbb{Z}} f^{-j} \partial \mathcal{R}\right)$ tal que

$$
x=\pi(\theta)=\pi\left(\theta^{\prime}\right) \in \Lambda \backslash \bigcup_{j \in \mathbb{Z}} f^{-j} \partial \mathcal{R}
$$

então $f^{j}(x) \in \operatorname{int} R_{\theta_{j}} \cap \operatorname{int} R_{\theta_{j}^{\prime}} \forall j \in \mathbb{Z}, \operatorname{assim} \theta_{j}=\theta_{j}^{\prime} \forall j \in \mathbb{Z}$.

iii) Como

$$
\pi^{-1}\left(\bigcup_{j \in \mathbb{Z}} f^{-j} \partial \mathcal{R}\right)=\bigcup_{j \in \mathbb{Z}} \pi^{-1} \circ f^{-j} \partial \mathcal{R}=\bigcup_{j \in \mathbb{Z}} \sigma^{-j} \circ \pi^{-1} \partial \mathcal{R}
$$

e desde que $\mu$ é invariante por $\sigma$, então é suficiente mostrar que $\mu\left(\pi^{-1} \partial \mathcal{R}\right)=0$. Como

$$
\partial \mathcal{R}=\partial^{s} \mathcal{R} \cup \partial^{u} \mathcal{R} \Rightarrow \pi^{-1}(\partial \mathcal{R})=\pi^{-1}\left(\partial^{s} \mathcal{R}\right) \cup \pi^{-1}\left(\partial^{u} \mathcal{R}\right)
$$

Afirmação: $\mu\left(\pi^{-1}\left(\partial^{s} \mathcal{R}\right)\right)=0$ e $\mu\left(\pi^{-1}\left(\partial^{u} \mathcal{R}\right)\right)=0$. De fato, observe que

$$
\mu\left(\pi^{-1}\left(\partial^{s} \mathcal{R}\right)\right)=\mu\left(\sigma \pi^{-1}\left(\partial^{s} \mathcal{R}\right)\right)=\mu\left(\pi^{-1} f \partial^{s} \mathcal{R}\right)
$$

por outro lado

$$
\sigma \pi^{-1} f \partial^{s} \mathcal{R}=\pi^{-1} f\left(f \partial^{s} \mathcal{R}\right) \subset \pi^{-1} f \partial^{s} \mathcal{R}
$$

pois, da Proposição 1.38 temos que $f\left(\partial^{s} \mathcal{R}\right) \subset \partial^{s} \mathcal{R}$, logo como $\mu$ é ergódica, tem-se

$$
\mu\left(\pi^{-1} f \partial^{s} \mathcal{R}\right)=0 \text { ou } 1 .
$$

Se $\mu\left(\pi^{-1} f \partial^{s} \mathcal{R}\right)=1$, e como $f \partial^{s} \mathcal{R}$ é fechado $\Rightarrow \Sigma_{A} \backslash \pi^{-1}\left(f \partial^{s} \mathcal{R}\right)$ é aberto, logo por hipótese temos $\mu\left(\Sigma_{A} \backslash \pi^{-1}\left(f \partial^{s} \mathcal{R}\right)\right)>0$ o qual é um absurdo.

iv) Sejam $U, V \subset \Sigma_{A}$ abertos não vazios, então tomando $\alpha \in U$ e $\beta \in V$ existe $N \in \mathbb{N}$ tal que

$$
U_{1}=\left\{\theta \in \Sigma_{A}: \theta_{j}=\alpha_{j} \text { para }|j| \leq N\right\} \subset U \mathrm{e}
$$




$$
V_{1}=\left\{\theta \in \Sigma_{A}: \theta_{j}=\beta_{j} \text { para }|j| \leq N\right\} \subset V
$$

do Lema 5.3, temos que $K_{N}(\theta)$ é um retângulo fechado, então

$$
\operatorname{int} f^{-n} R_{\theta_{j}}=f^{-n} \operatorname{int} R_{\theta_{j}}
$$

assim

$$
\begin{aligned}
& \emptyset \neq \operatorname{int}\left(K_{N}(\alpha)\right)=\bigcap_{-N}^{N} f^{-j} \operatorname{int} R_{\alpha_{j}}=U_{2} \\
& \emptyset \neq \operatorname{int}\left(K_{N}(\beta)\right)=\bigcap_{-N}^{N} f^{-j} \operatorname{int} R_{\beta_{j}}=V_{2}
\end{aligned}
$$

Afirmação: $\pi^{-1}\left(U_{2}\right) \subset U_{1}$ e $\pi^{-1}\left(V_{2}\right) \subset V_{1}$. De fato,

$$
\theta \in \pi^{-1}\left(U_{2}\right) \Rightarrow x=\pi(\theta) \in U_{2} \Rightarrow f^{j}(x) \in R_{\theta_{j}} \text { e } f^{j}(x) \in \operatorname{int} R_{\alpha_{j}}
$$

logo, $\theta_{j}=\alpha_{j}$ para $|j| \leq N$. De forma análoga se mostra $\pi^{-1}\left(V_{2}\right) \subset V_{1}$.

Por outro lado, como $f$ é topologicamente mixing existe $m_{0}$ tal que

$$
f^{m}\left(U_{2}\right) \cap V_{2} \neq \emptyset \text { para } m \geq m_{0}
$$

usando a afirmação e a última linha segue que

$$
\begin{aligned}
& \sigma^{m}(U) \cap V \supset \sigma^{m} \pi^{-1}\left(U_{2}\right) \cap \pi^{-1}\left(V_{2}\right)=\pi^{-1} f^{m}\left(U_{2}\right) \cap \pi^{-1}\left(V_{2}\right)=\pi^{-1}\left(f^{m}\left(U_{2}\right) \cap V_{2}\right) \neq \emptyset \\
& \text { para } m \geq m_{0} .
\end{aligned}
$$

Teorema 5.4 (R. Bowen): Seja $f: M \rightarrow M$ um difeomorfismo e $\Lambda \subset M$ um conjunto hiperbólico com estrutura de produto local. Se $\left.f\right|_{\Lambda}$ que é topologicamente mixing, então existem $\lambda>0$ e uma única medida de probabilidade $\rho$ em $\Lambda$ invariante por $f$ tais que

$$
h_{\text {top }}\left(\left.f\right|_{\Lambda}\right)=h_{\rho}\left(\left.f\right|_{\Lambda}\right)=\log \lambda
$$

Demonstração. Desde $\left.f\right|_{\Lambda}$ é transitivo segue-se do Teorema 1.34 que $\Lambda$ possui uma partição de Markov $\mathcal{R}=\left\{R_{1}, \cdots, R_{m}\right\}$ e da Proposição anterior temos que

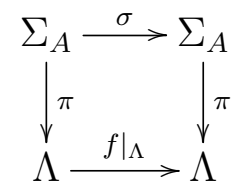


o diagrama comuta. Por outro lado como $\left.f\right|_{\Lambda}$ é topologicamente mixing segue da Proposição anterior e da Proposição 1.53 que $A$ é Aperiódica. Logo, pelo Teorema PerronFrobenius existe $\lambda>0$ autovalor dominante de $A$, assim pelo Teorema de Parry, existe $\nu$ uma única medida de Markov que é ergódica e positiva em abertos de $\Sigma_{A}$ tal que

$$
h_{\nu}(\sigma)=\log \lambda=h_{t o p}(\sigma)
$$

assim de (ii) e (iii) da Proposição anterior temos que $\pi$ é bijectiva em um conjunto de medida total. Se consideramos $\rho=\pi_{*} \nu$ então $\rho$ é uma medida de probabilidade em $\Lambda$ invariante por $\left.f\right|_{\Lambda}$, por tanto $(\sigma, \nu)$ e $\left(\left.f\right|_{\Lambda}, \rho\right)$ são ergodicamente equivalentes e segue-se da Proposição 1.43 que

$$
h_{u}(\sigma)=h_{\rho}\left(\left.f\right|_{\Lambda}\right)
$$

Por outro lado, desde que $\sigma$ e $\left.f\right|_{\Lambda}$ são semi-conjugados segue da Proposição 1.47 que

$$
h_{\text {top }}(\sigma) \geq h_{\text {top }}\left(\left.f\right|_{\Lambda}\right)
$$

Assim, de (5.1), (5.2), (5.3) e o Principio Variacional conclui-se a Prova do Teorema.

Exemplo 5.5: Do capítulo 4, temos que o Thom-Arnold map satisfaz com as hipótese do teorema anterior. Vamos construir uma partição de Markov para Thom-Arnold map. Se observa na figura 5.1 que temos uma partição de $\mathbb{T}^{2}$ em dois conjuntos $R_{1}, R_{2}$.

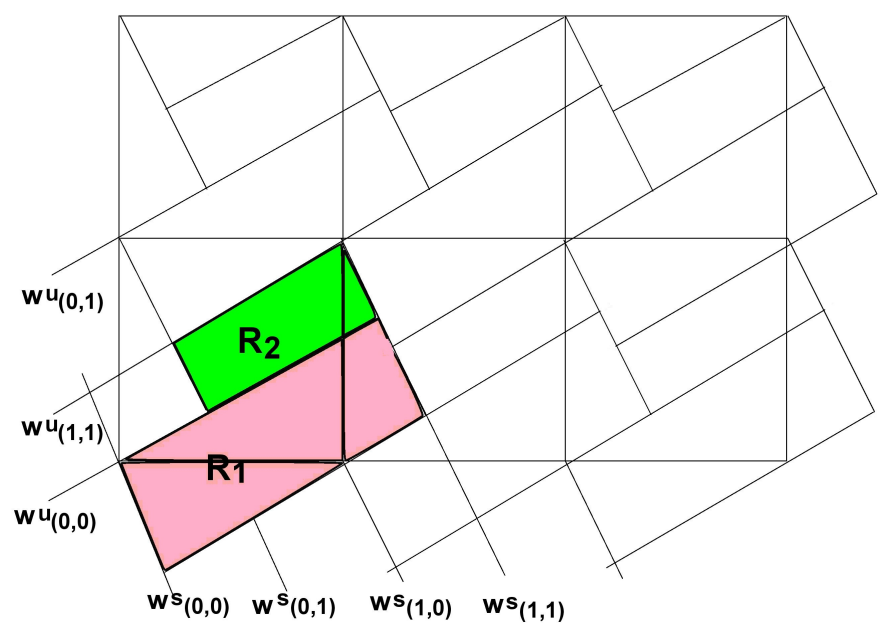

Figura 5.1: Partição de Thom-Arnold map em dois retângulos

A interseção de estos retângulos com seus imagens sobre $f$ fornecem 5 retângulos(ver figura 5.2). Se observa que $\left\{R_{1}, R_{2}\right\}$, não pode ser considerado uma partição de Markov 
para $\mathbb{T}^{2}$, pois $f\left(R_{1}\right)$ cruza mais de uma vez $R_{1}$. Logo o conjunto $\left\{P_{1}, P_{2}, P_{3}, P_{4}, P_{5}\right\}$ forma uma partição de Markov.

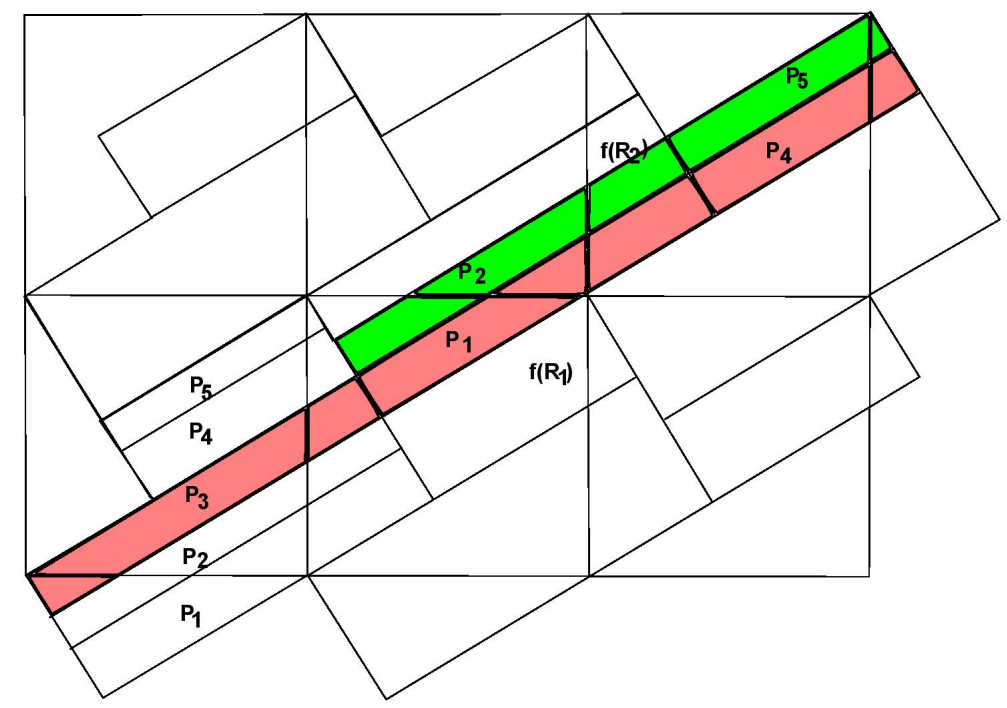

Figura 5.2: Imagem de $R_{1}$ e $R_{2}$

e temos que sua matriz de transição é

$$
A=\left(\begin{array}{lllll}
1 & 0 & 1 & 1 & 0 \\
1 & 0 & 1 & 1 & 0 \\
1 & 0 & 1 & 1 & 0 \\
0 & 1 & 0 & 0 & 1 \\
0 & 1 & 0 & 0 & 1
\end{array}\right)
$$

se observa que esta matriz é aperiódica. O autovalor dominante de $A$ é $\lambda=\frac{3+\sqrt{5}}{2}, \operatorname{logo}$ a entropia topologica de Thom-Arnold map é $\log \frac{3+\sqrt{5}}{2}$. Seja $u=(1, \lambda-2,1,1, \lambda-2)$ e $v=(1,1,1, \lambda-2, \lambda-2)$ o autovetor à esquerda e à direita respetivamente associado $\lambda$. 
Da observação 1.56 temos que a matriz estocástica é

$$
P=\frac{1}{\lambda}\left(\begin{array}{ccccc}
1 & 0 & 1 & \lambda-2 & 0 \\
1 & 0 & 1 & \lambda-2 & 0 \\
1 & 0 & 1 & \lambda-2 & 0 \\
0 & \frac{1}{\lambda-2} & 0 & 0 & 1 \\
0 & \frac{1}{\lambda-2} & 0 & 0 & 1
\end{array}\right)
$$

Exemplo 5.6: A ferradura de Smale, também satisfaz com as hipótese do teorema anterior. Uma partição de Markov da Ferradura de Smale é dada por $\mathcal{R}=\left\{R_{1}, R_{2}, R_{3}, R_{4}\right\}$ como mostra a figura 5.3

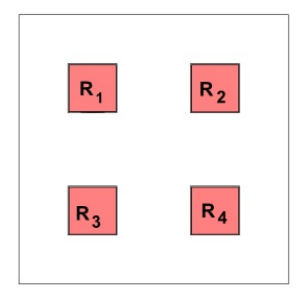

Figura 5.3: Partição de Markov da Ferradura de Smale

a matriz de transição é

$$
A=\left(\begin{array}{llll}
1 & 0 & 1 & 0 \\
1 & 0 & 1 & 0 \\
0 & 1 & 0 & 1 \\
0 & 1 & 0 & 1
\end{array}\right)
$$

Se observa que esta matriz é aperiódica. O autovalor dominante de $A$ é $\lambda=2$, logo a entropia topologica da ferradura de Smale é $\log 2$. Seja $u=(1,1,1,1)$ e $v=(1,1,1,1)$ o autovetor à esquerda e à direita respetivamente associado $\lambda$. Da observação 1.56 temos que a matriz estocástica é

$$
P=\frac{1}{2}\left(\begin{array}{llll}
1 & 0 & 1 & 0 \\
1 & 0 & 1 & 0 \\
0 & 1 & 0 & 1 \\
0 & 1 & 0 & 1
\end{array}\right)
$$

e o vetor de probabilidade é $p=\left(\frac{1}{4}, \frac{1}{4}, \frac{1}{4}, \frac{1}{4}\right)$.

\subsection{Medidas transversas e Sistemas dinâmicos}

Sejam $x, x^{\prime} \in \Lambda$ tal que $d\left(x, x^{\prime}\right)<\delta$ então podemos definir o homeomorfismo canônicos entre as partes de $W_{\epsilon}^{s}\left(x^{\prime}\right)$ e $W_{\epsilon}^{s}(x)$ denotado por $P_{x, x^{\prime}}^{s}$ definido pela projeção ao longo das 
variedades instáveis locais, isto é,

$$
\begin{array}{ccc}
P_{x, x^{\prime}}^{s}: W_{\epsilon}^{s}\left(x^{\prime}\right) & \longrightarrow W_{\epsilon}^{s}(x) \\
y & \mapsto[x, y]
\end{array}
$$

De forma análoga definimos o homeomorfismo $P_{x, x^{\prime}}^{u}$ entre as partes de $W_{\epsilon}^{u}\left(x^{\prime}\right)$ e $W_{\epsilon}^{u}(x)$ definido pela projeção ao longo das variedades estáveis locais, esto é,

$$
\begin{aligned}
P_{x, x^{\prime}}^{u}: W_{\epsilon}^{u}\left(x^{\prime}\right) & \longrightarrow W_{\epsilon}^{u}(x) \\
y & \mapsto[y, x]
\end{aligned}
$$

Observação 5.7: Da observação 1.56 temos que a medida de Parry, da demostração do teorema anterior é dado por

$$
\nu\left(\left[k, \alpha_{k}, \cdots, \alpha_{l}\right]\right)=\lambda^{-(l-k)} u_{\alpha_{k}} A_{\alpha_{k} \alpha_{k+1}} \cdots A_{\alpha_{l-1} \alpha_{l}} v_{\alpha_{l}}
$$

onde $\left[k, \alpha_{k}, \cdots, \alpha_{l}\right]=\left\{\theta \in \Sigma: \theta_{k}=\alpha_{k}, \cdots, \theta_{l}=\alpha_{l}\right\}$. Seja $\Sigma_{A}^{+}\left(\right.$resp. $\left.\Sigma_{A}^{-}\right)$definidos com $\Sigma_{A}$ como $\mathbb{Z}$ trocando por $\mathbb{Z}^{+}\left(\right.$resp. $\left.\mathbb{Z}^{-}\right)$. Definimos $\sigma^{+}: \Sigma_{A}^{+} \rightarrow \Sigma_{A}^{+}$e $\sigma^{-}: \Sigma_{A}^{-} \rightarrow \Sigma_{A}^{-}$de forma obvia. Assim, temos que existem medidas $\nu^{+}$em $\Sigma^{+}$e $\nu^{-}$em $\Sigma^{-}$tal que para cada $k \geq 0$ tem-se

$$
\begin{gathered}
\cdot \nu^{+}\left(\left\{\theta \in \Sigma^{+}: \theta_{0}=\alpha_{0}, \cdots, \theta_{k}=\alpha_{k}\right\}\right)=\lambda^{-k} A_{\alpha_{0} \alpha_{1}} \cdots A_{\alpha_{k-1} \alpha_{k}} v_{\alpha_{k}} \\
\cdot \nu^{-}\left(\left\{\theta \in \Sigma^{-}: \theta_{-k}=\alpha_{-k}, \cdots, \theta_{0}=\alpha_{0}\right\}\right)=\lambda^{-k} u_{\alpha_{-k}} A_{\alpha_{-k} \alpha_{-k+1}} \cdots A_{\alpha_{-1} \alpha_{0}}
\end{gathered}
$$

Teorema 5.8 (D. Ruelle, D. Sullivan): Seja $f: M \rightarrow M$ um difeomorfismo e $\Lambda \subset M$ um conjunto hiperbólico com estrutura de produto local. Se $\left.f\right|_{\Lambda}$ é topologicamente mixing, então para cada $x \in \Lambda$ existe uma medida $\mu_{x}^{s}$ em $W_{\epsilon}^{s}(x)$ e uma medida $\mu_{x}^{u}$ em $W_{\epsilon}^{u}(x)$ tal que:

i) $\operatorname{supp}\left(\mu_{x}^{s}\right) \subset W_{\epsilon}^{s}(x) \cap \Lambda, \operatorname{supp}\left(\mu_{x}^{u}\right) \subset W_{\epsilon}^{u}(x) \cap \Lambda$.

ii) $\operatorname{Se} d\left(x, x^{\prime}\right)<\delta$, então

$$
\left(P_{x, x^{\prime}}^{s}\right)_{*}\left(\left.\mu_{x^{\prime}}^{s}\right|_{W_{\delta}^{s}\left(x^{\prime}\right) \cap \Lambda}\right)=\left.\mu_{x}^{s}\right|_{P_{x, x^{\prime}}^{s}\left(W_{\delta}^{s}\left(x^{\prime}\right) \cap \Lambda\right)}
$$

analogamente

$$
\left(P_{x, x^{\prime}}^{u}\right)_{*}\left(\left.\mu_{x^{\prime}}^{u}\right|_{W_{\delta}^{u}\left(x^{\prime}\right) \cap \Lambda}\right)=\left.\mu_{x}^{u}\right|_{P_{x, x^{\prime}}^{u}}\left(W_{\delta}^{u}\left(x^{\prime}\right) \cap \Lambda\right)
$$

iii)

$$
\left.\left(f_{*}^{-1} \mu_{x}^{s}-\lambda^{-1} \mu_{f^{-1}(x)}^{s}\right)\right|_{W_{\epsilon}^{s}\left(f^{-1}(x)\right)}=0
$$

$e$

$$
\left.\left(f_{*} \mu_{x}^{u}-\lambda^{-1} \mu_{f(x)}^{u}\right)\right|_{W_{\epsilon}^{u}(f(x))}=0
$$


iv) Seja $[.,]:.\left(W_{\epsilon}^{u}(x) \cap \Lambda\right) \times\left(W_{\epsilon}^{s}(x) \cap \Lambda\right) \rightarrow \Lambda$ definida por $\{[y, z]\}=W_{\epsilon}^{s}(y) \cap W_{\epsilon}^{u}(z)$ então

$$
\left.[.,]_{*}\left(\mu_{x}^{u} \times \mu_{x}^{s}\right)\right|_{\left(W_{\epsilon}^{u}(x) \times W_{\epsilon}^{s}(x)\right) \cap \Lambda}=\left.\rho\right|_{\left(W_{\epsilon}^{u}(x) \times W_{\epsilon}^{s}(x)\right) \cap \Lambda}
$$

onde $\rho$ é a medida de Bowen em $\Lambda$.

Demonstração. Seja $y \in \Lambda$, como $\pi$ é sobrejetiva existe $\alpha \in \Sigma_{A}$ tal que

$$
y=\pi(\alpha)=\bigcap_{j \in \mathbb{Z}} f^{-j} R_{\alpha_{j}}
$$

definamos $\pi_{y, R_{\alpha_{0}}}^{+}:\left\{\theta \in \Sigma^{+}: \theta_{0}=\alpha_{0}\right\} \rightarrow W_{\epsilon}^{u}(y) \cap R_{\alpha_{0}}$ por

$$
\pi_{y, R_{\alpha_{0}}}^{+}(\theta)=\pi\left(\cdots, \alpha_{-2}, \alpha_{-1}, \alpha_{0}, \theta_{1}, \theta_{2}, \cdots\right)
$$

Afirmação I: $\left\{\pi_{y, R_{\alpha_{0}}}(\theta)\right\}=W_{\epsilon}^{u}(y) \cap\left(\bigcap_{j \in \mathbb{Z}^{+}} f^{-j} R_{\theta_{j}}\right)$. De fato, por definição temos

$$
\left\{\pi_{y, R_{\alpha_{0}}}^{+}(\theta)\right\} \subset W_{\epsilon}^{u}(y) \cap\left(\bigcap_{j \in \mathbb{Z}^{+}} f^{-j} R_{\theta_{j}}\right)
$$

Reciprocamente, seja $z \in W_{\epsilon}^{u}(y) \cap\left(\bigcap_{j \in \mathbb{Z}^{+}} f^{-j} R_{\theta_{j}}\right)$. Como

$$
y=\pi(\alpha) \Rightarrow y \in R_{\alpha_{0}} \cap f\left(R_{\alpha_{-1}}\right) \Rightarrow f^{-1}(y) \in R_{\alpha_{-1}} \cap f^{-1}\left(R_{\alpha_{0}}\right)
$$

e pela Proposição 1.37 temos

$$
f W^{u}\left(f^{-1}(y), R_{\alpha_{-1}}\right) \supset W^{u}\left(y, R_{\alpha_{0}}\right) \Rightarrow z \in f W^{u}\left(f^{-1}(y), R_{\alpha_{-1}}\right) \text { e } f^{-1}(z) \in R_{\alpha_{-1}}
$$

de forma análoga se prova que

$$
f^{-1}(z) \in f W^{u}\left(f^{-2}(y), R_{\alpha_{0}}\right) \text { e } f^{-2}(z) \in R_{\alpha_{-2}}
$$

pois $f^{-2}(y) \in R_{\alpha_{-2}} \cap f^{-1}\left(R_{\alpha_{-1}}\right)$. E em geral $f^{-j}(z) \in R_{\alpha_{-j}}$ para $j \geq 0$. Assim $\pi_{y, R_{\alpha_{0}}}^{+}(\theta)=z$ o que conclui a afirmação.

Agora definamos a seguinte medida em $W_{\epsilon}^{u}(y) \cap R_{\alpha_{0}}$

$$
\mu_{y, R_{\alpha_{0}}}^{u}:=\left.\left(\pi_{y, R_{\alpha_{0}}}^{+}\right)_{*} \nu^{+}\right|_{\left\{\theta \in \Sigma_{A}^{+}: \theta_{0}=\alpha_{0}\right\}}
$$

a medida $\mu_{y, R_{\alpha_{0}}}^{s}$ é definida semelhantemente em $W_{\epsilon}^{s}(y) \cap R_{\alpha_{0}}$.

Afirmação II: Dado $\theta \in\left\{\theta \in \Sigma_{A}^{+}: \theta_{0}=\alpha_{0}\right\}$ tem-se

$$
f \circ \pi_{y, R_{\alpha_{0}}}^{+}(\theta)=\pi_{y^{*}\left(R_{\theta_{1}}\right), R_{\theta_{1}}}^{+} \circ \sigma^{+}(\theta)
$$


para qualquer $y^{*}\left(R_{\theta_{1}}\right) \in R_{\theta_{1}} \cap f\left(W_{\epsilon}^{u}(y) \cap R_{\alpha_{0}}\right)$. De fato,

$$
\begin{aligned}
f \circ \pi_{y, R_{\alpha_{0}}}^{+}(\theta) & =f \circ \pi\left(\cdots, \alpha_{-2}, \alpha_{-1}, \alpha_{0}, \theta_{1}, \theta_{2}, \cdots\right) \\
& =\pi \circ \sigma\left(\cdots, \alpha_{-2}, \alpha_{-1}, \alpha_{0}, \theta_{1}, \theta_{2}, \cdots\right)
\end{aligned}
$$

Por outro lado, seja $y^{*}=y^{*}\left(R_{\theta_{1}}\right) \in R_{\theta_{1}} \cap f\left(W_{\epsilon}^{u}(y) \cap R_{\alpha_{0}}\right)$ arbitrário, então existe $z \in$ $W_{\epsilon}^{u}(y) \cap R_{\alpha_{0}}=W^{u}\left(y, R_{\alpha_{0}}\right)$, desde que $y=\pi(\alpha)=\bigcap_{j \in \mathbb{Z}} f^{-j} R_{\alpha_{j}}$ e fazendo as mesmas contas da prova da afirmação I, temos que

$$
z \in \bigcap_{j \leq 0} f^{-j} R_{\alpha_{j}} \Rightarrow y^{*}=f(z) \in \bigcap_{j \leq 0} f^{-j+1} R_{\alpha_{j}}
$$

Assim $y^{*}=\pi(\beta) \operatorname{com} \beta_{0}=\theta_{1}, \beta_{j}=\alpha_{j+1}$ para $j \leq-1$. Logo por definição tem-se

$$
\begin{aligned}
\pi_{y^{*}, R_{\alpha_{1}}}^{+} \circ \sigma^{+}(\theta) & =\pi_{y^{*}, R_{\alpha_{1}}}^{+}\left(\theta_{1}, \theta_{2}, \cdots\right) \\
& =\pi\left(\cdots, \beta_{-3}, \beta_{-2}, \beta_{-1}, \beta_{0}, \theta_{2}, \theta_{3}, \cdots\right) \\
& =\pi\left(\cdots, \alpha_{-2}, \alpha_{-1}, \alpha_{0}, \theta_{1}, \theta_{2}, \cdots\right)
\end{aligned}
$$

de (5.4) e (5.5) segue a afirmação.

Afirmação III: $\left.\left(\sigma^{+}\right)_{*} \nu^{+}\right|_{\left\{\theta \in \Sigma_{A}^{+}: \theta_{0}=\alpha_{0}, \theta_{1}=\alpha_{1}\right\}}=\left.\lambda^{-1} \nu^{+}\right|_{\left\{\theta \in \Sigma_{A}^{+}: \theta_{0}=\alpha_{1}\right\}}$.

De fato, é suficiente mostrar para cilindros

$$
\begin{aligned}
C & =\left\{\theta \in \Sigma_{A}^{+}: \theta_{1}=\beta_{1}, \cdots, \theta_{l}=\beta_{l}\right\} \\
\left.\lambda^{-1} \nu^{+}\right|_{\left\{\theta \in \Sigma_{A}^{+}: \theta_{0}=\alpha_{1}\right\}}(C) & =\lambda^{-1} \nu^{+}\left(\left\{\theta \in \Sigma_{A}^{+}: \theta_{0}=\alpha_{1}, \theta_{1}=\beta_{1}, \cdots, \theta_{l}=\beta_{l}\right\}\right) \\
& =\lambda^{-1} \lambda^{-l} A_{\alpha_{1} \beta_{1}} A_{\beta_{1} \beta_{2}} \cdots A_{\beta_{l-1} \beta_{l}} v_{\beta_{l}}
\end{aligned}
$$

Por outro lado, como $\left(\sigma^{+}\right)^{-1}(C)=\left\{\theta \in \Sigma_{A}^{+}: \theta_{2}=\beta_{1}, \cdots, \theta_{l+1}=\beta_{l}\right\}$, então

$$
\begin{aligned}
\left.\left(\sigma^{+}\right)_{*} \nu^{+}\right|_{\left\{\theta \in \Sigma_{A}^{+}: \theta_{0}=\alpha_{0}, \theta_{1}=\alpha_{1}\right\}}(C) & =\left.\nu^{+}\right|_{\left\{\theta \in \Sigma_{A}^{+}: \theta_{0}=\alpha_{0}, \theta_{1}=\alpha_{1}\right\}}\left(\left(\sigma^{+}\right)^{-1}(C)\right) \\
& =\nu^{+}\left(\left\{\theta \in \Sigma_{A}^{+}: \theta_{0}=\alpha_{1}, \theta_{1}=\alpha_{2}, \theta_{2}=\beta_{1}, \cdots, \theta_{l+1}=\beta_{l}\right\}\right) \\
& =\lambda^{-(l+1)} A_{\alpha_{0} \alpha_{1}} A_{\alpha_{1} \beta_{1}} A_{\beta_{1} \beta_{2}} \cdots A_{\beta_{l-1} \beta_{l}} v_{\beta_{l}}
\end{aligned}
$$

Como $A_{\alpha_{0} \alpha_{1}}=1$ pois $\alpha \in \Sigma_{A}$. Logo a afirmação segue de (5.6) e (5.7).

Afirmação IV: $f_{*} \mu_{y, R_{\alpha_{0}}}^{u}=\lambda^{-1} \sum_{\beta_{1}} A_{\alpha_{0} \beta_{1}} \mu_{y^{*}\left(R_{\beta_{1}}\right), R_{\beta_{1}}}^{u}$. De fato,

$$
\begin{aligned}
f_{*} \mu_{y, R_{\alpha_{0}}}^{u}(B) & =\mu_{y, R_{\alpha_{0}}}^{u}\left(f^{-1}(B)\right) \\
& =\left.\left(\pi_{y, R_{\alpha_{0}}}^{+}\right)_{*} \nu^{+}\right|_{\left\{\theta \in \Sigma_{A}^{+}: \theta_{0}=\alpha_{0}\right\}}\left(f^{-1} B\right) \\
& =\left.\nu^{+}\right|_{\left\{\theta \in \Sigma_{A}^{+}: \theta_{0}=\alpha_{0}\right\}}\left(\left(\pi_{y, R_{\alpha_{0}}}^{+}\right) f^{-1}(B)\right) \\
& =\left.\sum_{\beta_{1}} \nu^{+}\right|_{\left\{\theta \in \Sigma_{A}^{+}: \theta_{0}=\alpha_{0}, \theta_{1}=\beta_{1}\right\}}\left(f \circ \pi_{y, R_{\alpha_{0}}}^{+}\right)^{-1}(B)
\end{aligned}
$$


e das Afirmações (II) e (III), temos

$$
\begin{aligned}
f_{*} \mu_{y, R_{\alpha_{0}}}^{u}(B) & =\left.\sum_{\beta_{1}} \nu^{+}\right|_{\left\{\theta \in \Sigma_{A}^{+}: \theta_{0}=\alpha_{0}, \theta_{1}=\beta_{1}\right\}}\left(\pi_{y^{*}\left(R_{\beta_{1}}\right), R_{\beta_{1}}}^{+} \circ \sigma^{+}\right)^{-1}(B) \\
& =\sum_{\beta_{1}} \underbrace{\nu^{+}}_{\sum_{\left\{\theta \in \Sigma_{A}^{+}: \theta_{0}=\alpha_{0}, \theta_{1}=\beta_{1}\right\}}\left(\sigma^{+}\right)^{-1}} \circ\left(\pi_{y^{*}\left(R_{\beta_{1}}\right), R_{\beta_{1}}}^{+}\right)^{-1}(B) \\
& =\sum_{\beta_{1}, R_{\alpha_{0}} \cap f^{-1} R_{\beta_{1}}}^{\left.\nu^{+}\right|_{\left\{\theta \in \Sigma_{A}^{+}: \theta_{0}=\beta_{1}\right\}}\left(\pi_{y^{*}\left(R_{\beta_{1}}\right), R_{\beta_{1}}}^{+} \circ \sigma^{+}\right)^{-1}}(B) \\
& =A_{\alpha_{0} \beta_{1}} \lambda^{-1} \mu_{y^{*}\left(R_{\beta_{1}}\right), R_{\beta_{1}}}^{u}(B)
\end{aligned}
$$

o que prova a afirmação. Para cada $x \in \Lambda$, definamos $\mu_{x}^{u}$ em $W_{\epsilon}^{u}(x)$ como segue: para cada $R \subset \Lambda$ retângulo da partição de markov $\mathcal{R}$ de $\Lambda$ tal que $W_{\epsilon}^{u}(x) \cap R \neq \emptyset$ temos que

$$
\left.\mu_{x}^{u}\right|_{W_{\epsilon}^{u}(x) \cap R}=\mu_{y, R}^{u}
$$

para qualquer $y \in W_{\epsilon}^{u}(x) \cap R$. Para que $\mu_{x}^{u}$ esteja bem definida precisamos mostrar que

a) Se $y^{\prime} \in W_{\epsilon}^{u}(x) \cap R, y \neq y^{\prime} \Rightarrow \mu_{y, R}^{u}=\mu_{y^{\prime}, R}^{u}$

b) Se $W_{\epsilon}^{u}(x) \cap R \cap R^{\prime} \neq \emptyset \Rightarrow \mu_{y, R}^{u}=\mu_{y, R^{\prime}}^{u} \operatorname{em} W_{\epsilon}^{u}(x) \cap R \cap R^{\prime} \operatorname{com} y \in R \cap R^{\prime}$

da Proposição 1.29 temos $W_{\epsilon}^{u}(y) \cap R=W_{\epsilon}^{u}\left(y^{\prime}\right) \cap R=W_{\epsilon}^{u}(x) \cap R$, se $y=\pi(\alpha)$ e fazendo as mesmas contas da prova da afirmação (I) temos que $f^{-j} y^{\prime} \in R_{\alpha_{-j}}$ para $j \geq 0$ de onde segue que $\pi_{y^{\prime}, R}^{+}=\pi_{y, R}^{+}$o qual prova (a). A parte $(b)$ segue-se de $\mu_{y, R}^{u}\left(W_{\epsilon}^{u}(y) \cap \partial R \cap \partial R^{\prime}\right)=$ $\mu_{y, R^{\prime}}^{u}\left(W_{\epsilon}^{u}(y) \cap \partial R \cap \partial R^{\prime}\right)=0$, mas isto segue de (5.8). Agora mostraremos que estas medidas assim definidas satisfazem as condições do teorema:

ii) Para mostrar que

$$
\left(P_{x, x^{\prime}}^{u}\right)_{*}\left(\left.\mu_{x^{\prime}}^{u}\right|_{W_{\delta}^{u}\left(x^{\prime}\right) \cap \Lambda}\right)=\left.\mu_{x}^{u}\right|_{P_{x, x^{\prime}}^{u}\left(W_{\delta}^{u}\left(x^{\prime}\right) \cap \Lambda\right)}
$$

pela forma como foi definido a medida só es preciso mostrar

$$
\left(P_{y, y^{\prime}}^{u}\right)_{*} \mu_{y^{\prime}, R^{\prime}}^{u}=\mu_{y, R}^{u} \operatorname{em}\left(W_{\epsilon}^{u}(y) \cap R\right) \cap P_{y, y^{\prime}}^{u}\left(W_{\epsilon}^{u}\left(y^{\prime}\right) \cap R^{\prime}\right)
$$

seja $y=\pi(\alpha)$ e $y^{\prime}=\pi\left(\alpha^{\prime}\right) ; R=R_{\alpha_{0}}$ e $R^{\prime}=R_{\alpha_{0}^{\prime}}$.

caso 1: Se $R=R^{\prime}$

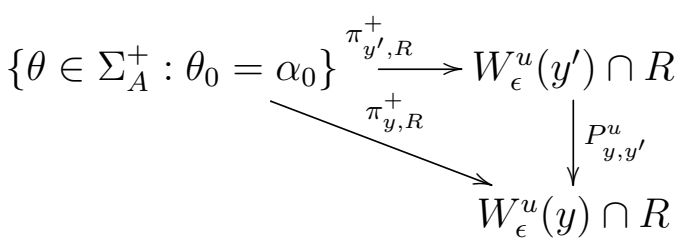


Afirmação: $P_{y, y^{\prime}}^{u} \circ \pi_{y^{\prime}, R}^{+}=\pi_{y, R}^{+}$. De fato,

$$
\begin{aligned}
P_{y, y^{\prime}}^{u} \circ \pi_{y^{\prime}, R}^{+}(\theta) & =P_{y, y^{\prime}}^{u}(\underbrace{\pi\left(\cdots, \alpha_{-2}^{\prime}, \alpha_{-1}^{\prime}, \alpha_{0}^{\prime}, \theta_{0}, \theta_{2}, \cdots\right)}_{z}) \\
& =W_{\epsilon}^{s}(z) \cap W_{\epsilon}^{u}(y)=w
\end{aligned}
$$

Por outro lado, se $z^{\prime}=\pi_{y, R}^{+}(\theta) \Rightarrow z^{\prime} \in W_{\epsilon}^{s}(z) \cap W_{\epsilon}^{u}(y)$, pois, por definição $z^{\prime} \in W_{\epsilon}^{u}(y)$. Como $z^{\prime}=\pi_{y, R}^{+}(\theta)=\pi\left(\cdots, \alpha_{-2}, \alpha_{-1}, \alpha_{0}, \theta_{0}, \theta_{2}, \cdots\right)$ e $z=\pi\left(\cdots, \alpha_{-2}^{\prime}, \alpha_{-1}^{\prime}, \alpha_{0}^{\prime}, \theta_{0}, \theta_{2}, \cdots\right)$, então $f^{j}(z), f^{j}\left(z^{\prime}\right) \in R_{\theta_{j}}$ para $j \leq 0$, e desde que o diâmetro de $R_{\theta_{j}}$ é menor que $\epsilon$ segue que $z^{\prime} \in W_{\epsilon}^{s}(z)$, logo $z^{\prime}=w$ o qual prova a afirmação.

E segue imediatamente da afirmação o caso 1.

caso 2: Se $R \neq R^{\prime}$

$$
\begin{aligned}
& \left\{\theta \in \Sigma_{A}^{+}: \theta_{0}=\alpha_{0}^{\prime}\right\}^{\pi_{y^{\prime}, R^{\prime}}^{+}} \longrightarrow W_{\epsilon}^{u}\left(y^{\prime}\right) \cap R^{\prime} \\
& \qquad \theta P_{y, y^{\prime}}^{u} \\
& \left\{\theta \in \Sigma_{A}^{+}: \theta_{0}=\alpha_{0}\right\} \stackrel{\pi_{y, R}^{+}}{\longrightarrow} W_{\epsilon}^{u}(y) \cap R
\end{aligned}
$$

Seja $Y^{+}$o conjunto de pontos de $\theta \in \Sigma_{A}^{+}$tal que $\theta_{0}=\alpha_{0}^{\prime}$ e existe $\beta \in \Sigma_{A}^{+}$tal que
a) $\beta_{0}=\alpha_{0}$
b) $P_{y, y^{\prime}}^{u} \circ \pi_{y^{\prime}, R^{\prime}}^{+}(\theta)=\pi_{y, R}^{+}(\beta)$
c) $\theta_{i} \neq \beta_{i} \forall i \in \mathbb{Z}^{+}$

neste caso é suficiente mostrar que

$$
\nu^{+}\left(Y^{+}\right)=0
$$

$\nu^{+}\left(Y^{+}\right)=0$ segue imediatamente se $\nu(Y)=0$ onde $Y$ é o conjunto de pontos $\theta \in \Sigma_{A}$ tal que $\theta_{0}=\alpha_{0}^{\prime}$ e existe $\beta \in \Sigma_{A}^{+}$tal que
a) $\beta_{0}=\alpha_{0}$
b) $P_{y, y^{\prime}}^{u} \circ \pi(\theta)=\pi_{y, R}^{+}(\beta)$
c) $\theta_{i} \neq \beta_{i} \forall i \in \mathbb{Z}^{+}$

agora mostraremos que $\nu(Y)=0$. Seja $z \in \pi(Y) \Rightarrow \exists \theta \in Y$ tal que $z=\pi(\theta)$, $\operatorname{logo} \exists \beta \in \Sigma_{A}^{+}$tal que $\forall k \in \mathbb{Z}^{+} \theta_{k} \neq \beta_{k}, f^{k}(z) \in R_{\theta_{k}}$ e $\lim _{k \rightarrow \infty} d\left(f^{k} z, R_{\beta_{k}}\right)=0$ uniformemente em $z$, pois $P_{y, y^{\prime}}^{u}(z)=\pi_{y, R}^{+}(\beta) \Rightarrow f^{k}\left(P_{y, y^{\prime}}^{u}(z)\right) \in R_{\beta_{k}} k \geq 0 \Rightarrow$ $d\left(f^{k}(z), R_{\beta_{k}}\right) \leq d\left(f^{k}(z), f^{k}\left(P_{y, y^{\prime}}^{u}(z)\right)\right)<\epsilon \forall k \geq 0$

Como $\rho=\pi_{*} \nu$ é invariante por $f$ segue

$$
\nu(Y)=\rho(\pi(Y))=\lim \rho\left(f^{k}(\pi(Y))\right)
$$


Afirmação: Para $k$ suficientemente grande $f^{k} z \in \partial R_{\theta_{k}}$. De fato, vamos supor que $f^{k} z \notin \partial R_{\theta_{k}} \Rightarrow \in \operatorname{int} R_{\theta_{k}} \Rightarrow \exists r>0$ tal que $B\left(f^{k} z, r\right) \subset R_{\theta_{k}}$. Como $\lim _{k \rightarrow \infty} d\left(f^{k} z, R_{\beta_{k}}\right)=0 \Rightarrow d\left(f^{k} z, R_{\beta_{k}}\right)<\frac{r}{2}$ para $k$ suficientemente grande. Logo, para $\frac{r}{2}, \exists y \in R_{\beta_{k}}$ tal que $y \notin R_{\theta_{k}} \mathrm{e}$

$$
d\left(f^{k} z, y\right)<d\left(f^{k} z, R_{\beta_{k}}\right)+\frac{r}{2}
$$

assim, $y \in R_{\theta_{k}}$ o qual é uma contradição. Por tanto segue-se a afirmação.

Segue da afirmação que $f^{k}(\pi(Y)) \subset \partial \mathcal{R}$ para $k$ suficientemente grande, e temos pela Proposição 5.2 que $\nu\left(\pi^{-1}\left(\bigcup_{j \in \mathbb{Z}} f^{-j} \partial \mathcal{R}\right)\right)=0$. Então de (5.9) segue-se que

$$
\nu(Y)=\rho(\pi(Y))=\lim \rho\left(f^{k}(\pi(Y))\right)=0 .
$$

iii) Pela forma como foi definido a medida $\mu_{x}^{u}$, temos que mostras que se $R$ é um retângulo tal que $W_{\epsilon}^{u}(x) \cap R \neq \emptyset$ e se $y \in W_{\epsilon}^{u}(x) \cap R$ então

$$
f_{*} \mu_{y, R}^{u}=\lambda^{-1} \sum_{R \cap f^{-1} R^{\prime} \neq \emptyset} \mu_{y^{*}, R^{\prime}}^{u}
$$

com $y^{*} \in R^{\prime} \cap f\left(W^{u}(y, R)\right)$, mas isto já foi provado na Afirmação IV.

iv) Seja $R$ um retângulo da partição de Markov $\mathcal{R}$ tal que $W_{\epsilon}^{u}(x) \cap W_{\epsilon}^{s}(x) \cap R \neq \emptyset \mathrm{e}$ seja $y \in W_{\epsilon}^{u}(x) \cap W_{\epsilon}^{s}(x) \cap R$, temos que mostrar que

$$
[,]_{*}\left(\mu_{y, R}^{u} \times \mu_{y, R}^{s}\right)=\rho \operatorname{em} R \cap\left(W_{\epsilon}^{u}(x) \times W_{\epsilon}^{s}(x)\right)
$$

Seja $y=\pi(\alpha)$ com $\alpha \in \Sigma_{A}$ e $R=R_{\alpha_{0}}$. Definamos a aplicação $\Phi:\left\{\theta \in \Sigma_{A}^{+}: \theta_{0}=\alpha_{0}\right\} \times\left\{\theta \in \Sigma_{A}^{-}: \theta_{0}=\alpha_{0}\right\} \rightarrow\left\{\theta \in \Sigma_{A}: \theta_{0}=\alpha_{0}\right\}$ por

$$
\Phi\left(\left(\theta_{0}, \theta_{1}, \cdots\right) \times\left(\cdots, \theta_{-1}, \theta_{0}\right)\right)=\left(\cdots, \theta_{-1}, \theta_{0}, \theta_{1}, \cdots\right)
$$

Afirmação: $\Phi_{*}\left(\left.\nu^{+}\right|_{\left\{\theta \in \Sigma_{A}^{+}: \theta_{0}=\alpha_{0}\right\}} \times\left.\nu^{-}\right|_{\left\{\theta \in \Sigma_{A}^{-}: \theta_{0}=\alpha_{0}\right\}}\right)=\left.\nu\right|_{\left\{\theta \in \Sigma_{A}: \theta_{0}=\alpha_{0}\right\}}$. De fato, é suficiente provar para cilindros. Seja o cilindro

$$
\begin{gathered}
B=\left\{\theta \in \Sigma_{A}: \theta_{-k}=\alpha_{-k}, \cdots, \theta_{0}=\alpha_{0}, \cdots, \theta_{l}=\alpha_{l}\right\} \\
\Rightarrow \Phi^{-1}(B)=\left\{\theta \in \Sigma_{A}^{+}: \theta_{0}=\alpha_{0}, \cdots, \theta_{l}=\alpha_{l}\right\} \times\left\{\theta \in \Sigma_{A}^{-}: \theta_{-k}=\alpha_{-k}, \cdots, \theta_{0}=\alpha_{0}\right\}
\end{gathered}
$$

da Observacao 5.7 e (5.10) segue que 


$$
\begin{aligned}
\Phi_{*} & \left(\left.\nu^{+}\right|_{\left\{\theta \in \Sigma_{A}^{+}: \theta_{0}=\alpha_{0}\right\}} \times\left.\nu^{-}\right|_{\left\{\theta \in \Sigma_{A}^{-}: \theta_{0}=\alpha_{0}\right\}}\right)(B)=\left.\nu^{+}\right|_{\left\{\theta \in \Sigma_{A}^{+}: \theta_{0}=\alpha_{0}\right\}} \times\left.\nu^{-}\right|_{\left\{\theta \in \Sigma_{A}^{-}: \theta_{0}=\alpha_{0}\right\}}\left(\Phi^{-1}(B)\right) \\
& =\nu^{+}\left(\left\{\theta \in \Sigma_{A}^{+}: \theta_{0}=\alpha_{0}, \cdots, \theta_{l}=\alpha_{l}\right\}\right) \nu^{-}\left(\left\{\theta \in \Sigma_{A}^{-}: \theta_{-k}=\alpha_{-k}, \cdots, \theta_{0}=\alpha_{0}\right\}\right) \\
& =\lambda^{-l} A_{\alpha_{0} \alpha_{1}} \cdots A_{\alpha_{l-1} \alpha_{l}} v_{\alpha_{l}} \lambda^{-k} u_{\alpha_{-k}} A_{\alpha_{-k} \alpha_{-k+1}} \cdots A_{\alpha_{1} \alpha_{0}} \\
& =\lambda^{-(l+k)} u_{\alpha_{-k}} A_{\alpha_{-k} \alpha_{-k+1}} \cdots A_{\alpha_{l-1} \alpha_{l}} v_{\alpha_{l}} \\
& =\nu(B)
\end{aligned}
$$

o que prova a afirmação. Por outro lado, vemos que o seguente diagrama comuta

$$
\begin{aligned}
& \left\{\theta \in \Sigma_{A}^{+}: \theta_{0}=\alpha_{0}\right\} \times\left\{\theta \in \Sigma_{A}^{-}: \theta_{0}=\alpha_{0}\right\}^{\Phi} \longrightarrow\left\{\theta \in \Sigma_{A}: \theta_{0}=\alpha_{0}\right\} \\
& \left(W_{\epsilon}^{u}(y) \cap R_{\alpha_{0}}\right) \stackrel{\downarrow}{\pi_{y, R_{\alpha_{0}}}^{+} \times \pi_{y, R_{\alpha_{0}}}^{-}} \times\left(W_{\epsilon}^{s}(y) \cap R_{\alpha_{0}}\right) \stackrel{[,]}{\longrightarrow} R_{\alpha_{0}}
\end{aligned}
$$

i.e., $\pi \circ \Phi=[,] \circ\left(\pi_{y, R_{\alpha_{0}}}^{+} \times \pi_{y, R_{\alpha_{0}}}^{-}\right)$. De fato,

$$
\pi \circ \Phi\left(\theta_{0}, \theta_{1}, \cdots\right) \times\left(\cdots, \theta_{-1}, \theta_{0}\right)=\pi\left(\cdots, \theta_{-1}, \theta_{0}, \theta_{1}, \cdots\right)
$$

Por outro lado

$$
\begin{aligned}
& {[,] \circ\left(\pi_{y, R_{\alpha_{0}}}^{+} \times \pi_{y, R_{\alpha_{0}}}^{-}\right) }\left(\theta_{0}, \theta_{1}, \cdots\right) \times\left(\cdots, \theta_{-1}, \theta_{0}\right)= \\
&=[,](\underbrace{\pi_{y, R_{\alpha_{0}}}^{+}\left(\theta_{0}, \theta_{1}, \cdots\right)}_{z_{1}} \times \underbrace{\pi_{y, R_{\alpha_{0}}}^{-}\left(\cdots, \theta_{-1}, \theta_{0}\right)}_{z_{2}}) \\
&=\left[z_{1}, z_{2}\right]=W_{\epsilon}^{s}\left(z_{1}\right) \cap W_{\epsilon}^{u}\left(z_{2}\right)
\end{aligned}
$$

se $w=\pi\left(\cdots, \theta_{-1}, \theta_{0}, \theta_{1}, \cdots\right) \Rightarrow w \in W_{\epsilon}^{s}\left(z_{1}\right) \cap W_{\epsilon}^{u}\left(z_{2}\right)$, pois,

$\pi\left(\cdots, \theta_{-1}, \theta_{0}, \theta_{1}, \cdots\right)=\bigcap_{j \in \mathbb{Z}} f^{-j} R_{\theta_{j}} \Rightarrow f^{j}(w) \in R_{\theta_{j}} \forall j \in \mathbb{Z}$. Mas da Afirmação I, temos que $z_{1}=W_{\epsilon}^{u} \cap\left(\bigcap_{j \in \mathbb{Z}^{+}} f^{-j} R_{\theta_{j}}\right) \Rightarrow f^{j}\left(z_{1}\right) \in R_{\theta_{j}}$ para $j \geq 0$. Então $f^{j}(w), f^{j}\left(z_{1}\right) \in R_{\theta_{j}}$ para $j \geq 0$ e desde que o diâmetro dos retângulos é menor que $\epsilon$ temos que $d\left(f^{j}(w), f^{j}\left(z_{1}\right)\right)<\epsilon$ para $j \geq 0 \Rightarrow w \in W_{\epsilon}^{s}\left(z_{1}\right)$. De forma análoga se prova que $w \in W_{\epsilon}^{u}\left(z_{2}\right)$. Como $W_{\epsilon}^{s}\left(z_{1}\right)$ e $W_{\epsilon}^{u}\left(z_{2}\right)$ se intersectam em um unico ponto segue que $w=W_{\epsilon}^{s}\left(z_{1}\right) \cap W_{\epsilon}^{u}\left(z_{2}\right)$. Assim de (5.11) e (5.12) segue que o diagrama comuta.

Da afirmação anterior e do fato que o diagrama anterior comuta segue-se que

$$
\begin{aligned}
{[,]_{*}\left(\mu_{y, R}^{u} \times \mu_{y, R}^{s}\right)(B) } & =\left(\mu_{y, R}^{u} \times \mu_{y, R}^{s}\right)\left([,]^{-1} B\right) \\
& =\left.\left(\pi_{y, R_{\alpha_{0}}}^{+}\right)_{*} \nu^{+}\right|_{\left\{\theta \in \Sigma_{A}^{+}: \theta_{0}=\alpha_{0}\right\}} \times\left.\left(\pi_{y, R_{\alpha_{0}}}^{+}\right)_{*} \nu^{-}\right|_{\left\{\theta \in \Sigma_{A}^{-}: \theta_{0}=\alpha_{0}\right\}}\left([,]^{-1} B\right) \\
& =\left(\left.\nu^{+}\right|_{\left\{\theta \in \Sigma_{A}^{+}: \theta_{0}=\alpha_{0}\right\}} \times\left.\nu^{-}\right|_{\left\{\theta \in \Sigma_{A}^{-}: \theta_{0}=\alpha_{0}\right\}}\right)\left(\pi_{y, R_{\alpha_{0}}} \times \pi_{y, R_{\alpha_{0}}}^{-1} \circ\left([,]^{-1} B\right)\right. \\
& =\left(\left.\nu^{+}\right|_{\left\{\theta \in \Sigma_{A}^{+}: \theta_{0}=\alpha_{0}\right\}} \times\left.\nu^{-}\right|_{\left\{\theta \in \Sigma_{A}^{-}: \theta_{0}=\alpha_{0}\right\}}\right) \Phi^{-1} \circ \pi^{-1} B \\
& =\nu\left(\pi^{-1} B\right) \\
& =\rho(B)
\end{aligned}
$$

o que conclui a prova do item (iv). 
O seguente resultado relaciona as correntes e os sistemas dinâmicos. Este resultado é nosso segundo objetivo deste trabalho.

Teorema 5.9 (D. Ruelle, D. Sullivan): Se $f: M \rightarrow M$ é um difeomorfismo de Anosov topologicamente mixing, preservando a orientação das folhas estáveis e folhas instáveis, então existem $C^{s} k$-corrente e $C^{u}(n-k)$-corrente tal que

i) $C^{s}$ e $C^{u}$ são fechados e definem classes de cohomologia de DeRham $\left[C^{s}\right]$ e $\left[C^{u}\right]$ em $H^{n-k}(M)$ e $H^{k}(M)$ respectivamente, onde $k$ é a dimensão das variedades estáveis e $n$ é a dimensão da variedade $M$.

ii) $f_{*} C^{s}=\frac{1}{\lambda} C^{s}$ e $f_{*} C^{u}=\lambda C^{u}$ onde $\log \lambda$ é a entropia topológica de $f$.

Demonstração. Desde que $f: M \rightarrow M$ é topologicamente mixing, temos do Teorema anterior que para cada $x \in M$ existe uma medida $\mu_{x}^{s}$ em $W_{\epsilon}^{s}(x)$ e uma medida $\mu_{x}^{u}$ em $W_{\epsilon}^{u}(x)$. Denotemos por $\mathcal{W}^{s}$ a folheação gerada pelas variedades estáveis $W^{s}(x)$ e por $\mathcal{W}^{u}$ a folheação gerada pelas variedades instáveis $W^{u}(x), x \in M$. Vamos supor que a dimensão da folheação $\mathcal{W}^{s}$ é $k$. Como $M$ tem estrutura de produto local, temos que $W_{\epsilon}^{s}(x) \times W_{\epsilon}^{u}(x)$ é uma vizinhança aberta. Por tanto, se $x, x^{\prime} \in M$ tal que $d\left(x, x^{\prime}\right)<\delta$ os homeomorfismos locais $P_{x, x^{\prime}}^{u}: W_{\epsilon}^{u}\left(x^{\prime}\right) \rightarrow W_{\epsilon}^{u}(x)$ geram o pseudorgrupo de holonomia $\Gamma$ de $\mathcal{W}^{s}$ (ver definição 2.3). Logo pelo item (ii) do Teorema anterior, as medidas $\mu_{x}^{u}$ definem uma $\mu_{s}$ medida transversa invariante por holonomia, no espaço transverso de $\mathcal{W}^{s}$, assim pelo Teorema 3.30 temos que $\mu_{s}$ induz uma $k$-corrente que denotamos por $C^{s}$. De forma análoga se mostra a existência de $C^{u}$ uma $(n-k)$-corrente. Agora mostraremos que as correntes $C^{s}$ e $C^{u}$ satisfazem as condições do teorema.

i) Pela Proposição 3.29 temos que $C^{s}$ é fechado; i.e.,

$$
\left[C^{s}\right] \in H_{k}^{D R}(M) \simeq H^{n-k}(M) \simeq\left(H^{k}\right)^{*}
$$

ii) Desde que $W_{\epsilon}^{s}(x) \times W_{\epsilon}^{u}(x)$ é um aberto, podemos escolher um conjunto finito de pontos $\left\{x_{1}, x_{2}, \cdots, x_{m}\right\}$ tal que $f\left(W_{\epsilon}^{s}\left(x_{i}\right) \times W_{\delta_{1}}^{u}\left(x_{i}\right)\right)=W_{\delta_{2}}^{s}\left(f\left(x_{i}\right)\right) \times W_{\epsilon}^{u}\left(f\left(x_{i}\right)\right)$ e $\{\underbrace{W_{\epsilon}^{s}\left(x_{i}\right) \times W_{\delta_{1}}^{u}\left(x_{i}\right)}_{U_{i}}\}_{i=1}^{m},\{\underbrace{W_{\delta_{2}}^{s}\left(f\left(x_{i}\right)\right) \times W_{\epsilon}^{u}\left(f\left(x_{i}\right)\right)}_{U_{i}^{\prime}}\}_{i=1}^{m}$ são coberturas de $M$. Seja $\left\{\lambda_{i}\right\}_{i}$ uma partição da unidade subordinada a $\left\{U_{i}^{\prime}\right\}_{i}$, então $\left\{\beta_{i}=\lambda_{i} \circ f\right\}_{i}$ é uma partição da unidade subordinada a $\left\{U_{i}\right\}_{i}$. Consideremos a seguente função $g$ : 
$W_{\epsilon}^{u}\left(f\left(x_{i}\right)\right) \rightarrow \mathbb{R}$ definido por

$$
g(y)=\int_{W_{\delta_{2}}^{s}(z)} \lambda_{i} \omega
$$

onde $\omega$ é uma $k$-forma em $M$. Desde que $f_{*} \mu_{x_{i}}^{u}=\lambda^{-1} \mu_{f x_{i}}^{u}$ temos que

$$
\begin{aligned}
& \int_{W_{\delta_{1}}^{u}\left(x_{i}\right)}(\underbrace{}_{\left.\int_{W_{\epsilon}^{s}(y)}^{\int_{\delta_{2}(f y)} f_{i}^{*}\left(\lambda_{i} \omega\right)}\right) d \mu_{x_{i}}^{u}}=\int_{W_{\delta_{1}}^{u}\left(x_{i}\right)} g \circ f(y) d \mu_{x_{i}}^{u} \\
&=\int_{W_{\epsilon}^{u}\left(f\left(x_{i}\right)\right)} g(z) d\left(f_{*} \mu_{x_{i}}^{u}\right)(z) \\
&=\lambda^{-1} \int_{W_{\epsilon}^{u}\left(f\left(x_{i}\right)\right)} g(z) d \mu_{f x_{i}}^{u}(z) \\
&=\lambda^{-1} \int_{W_{\epsilon}^{u}\left(f\left(x_{i}\right)\right)}\left(\int_{W_{\delta_{2}}^{s}(z)} \lambda_{i} \omega\right) d \mu_{f x_{i}}^{u}(z)
\end{aligned}
$$

assim temos que

$$
\begin{aligned}
\underbrace{\sum_{i} \int_{W_{\delta_{1}}^{u}\left(x_{i}\right)}\left(\int_{W_{\epsilon}^{s}(y)}\left(\lambda_{i} \circ f\right) f^{*} \omega\right) d \mu_{x_{i}}^{u}(y)}_{\left\langle C^{s}, f^{*}(\omega)\right\rangle} & =\sum_{i} \underbrace{\int_{W_{\delta_{1}}^{u}\left(x_{i}\right)}\left(\int_{W_{\epsilon}^{s}(y)} f^{*}\left(\lambda_{i} \omega\right)\right) d \mu_{x_{i}}^{u}(y)} \\
& =\sum_{i} \lambda^{-1} \int_{W_{\epsilon}^{u}\left(f\left(x_{i}\right)\right)}\left(\int_{W_{\delta_{2}}^{s}(z)} \lambda_{i} \omega\right) d \mu_{f x_{i}}^{u}(z) \\
& =\lambda^{-1} \underbrace{\sum_{i} \int_{W_{\epsilon}^{u}\left(f\left(x_{i}\right)\right)}\left(\int_{W_{\delta_{2}}^{s}(z)} \lambda_{i} \omega\right) d \mu_{f x_{i}}^{u}(z)}_{\left\langle C^{s}, \omega\right\rangle}
\end{aligned}
$$

logo, da definição 3.18 segue-se $f_{*} C^{s}=\lambda^{-1} C^{s}$. De forma análoga se mostra que $f_{*} C^{u}=\lambda C^{u}$. 


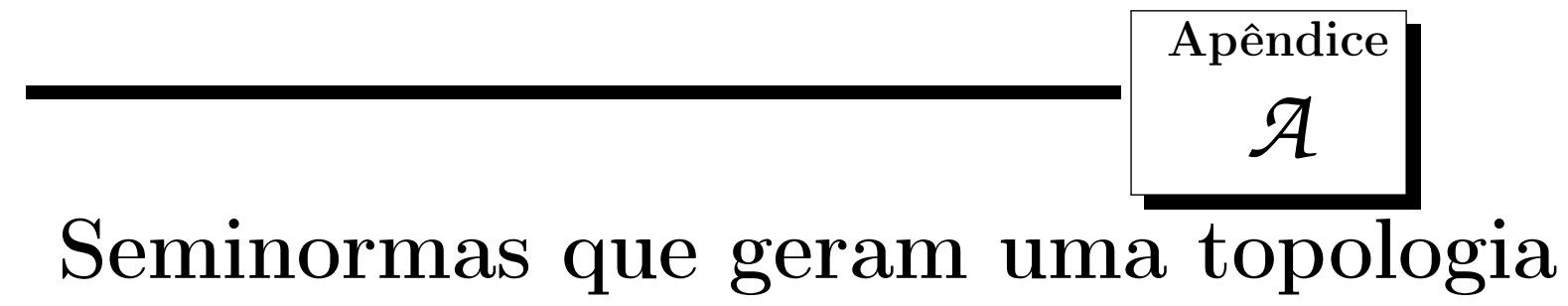

Um espaço vetorial topológico $X$ é chamado localmente convexo, se existe uma base de vizinhanças $\left\{U_{\alpha}\right\}$ de 0 de conjuntos convexos, equilibrados $(c x \in U$ se $x \in U$ e $|c|<1)$ e absorção(se $x \in X$ então existe $t>0$ tal que $x \in t U$ ). Uma seminorma em um espaço vetorial $X$ é uma aplicação $p: X \rightarrow \mathbb{R}$ tal que

1. $p(c x)=|c| p(x), c \in \mathbb{R}, x \in X$

2. $p(x+y) \leq p(x)+p(y), x, y \in X$

Uma família $\mathcal{P}$ de seminormas em $X$ é chamado de separação, se para cada $x \in X$, existe $p \in \mathcal{P}$ tal que $p(x) \neq 0$. Seja dado uma tal família e seja $B$ a coleção dos conjuntos da forma

$$
\{x: p(x)<\epsilon \mathrm{e} \epsilon>0\}
$$

e da interseção de um numero finito de conjuntos de tais conjuntos. Então não é difícil provar que $B$ é uma base de vizinhanças de 0 de um espaço vetorial topológico localmente convexo em $X$. Esta topologia é chamada topologia gerada pela seminormas pertencentes a $\mathcal{P}$. Reciprocamente, seja $X$ um espaço vetorial topológico localmente convexo. Se $U$ é uma vizinhança de 0 convexo, equilibrados e absorção, então

$$
p_{U}(x)=\inf \{t>0: x \in t U\}
$$

é chamado a funcional de Minkowski de $U$, é fácil mostrar que esto é uma seminorma e deduz-se que a topologia de $X$ pode ser gerado pela família de estas seminormas.

Proposição A.1: Seja X um espaço vetorial topológico localmente convexo, e seja $\mathcal{P}$ uma família de seminormas que geram a topologia de $X$. Então a semi-norma $q: X \rightarrow$ 
$\mathbb{R}$ é continua se, e somente se, existem um numero finito de semi-normas $p_{1}, \cdots, p_{N}$ pertencentes a $\mathcal{P}$, e uma constante $C>0$ tal que

$$
q(x) \leq C \max \left\{p_{1}(x), \cdots, p_{N}(x)\right\}, x \in X
$$

Demonstração. Se $q$ é continua, então $q^{-1}(-1,1)$ é uma vizinhança aberta de 0 , e também contém uma interseção finita de conjuntos da forma (A.1), e existem semi-normas $p_{1}, \cdots, p_{N} \in \mathcal{P}$, e números positivos $\epsilon_{1}, \cdots, \epsilon_{N} ;$ tal que $q(y)<1$ se $p_{j}(y)<\epsilon_{j}$ para $j=1, \cdots, N$. Seja $x \in X$ qualquer, definimos $y=t x$, onde $t>0$, então pode-se ver que

$$
t q(x)<1 \text { se } t p_{j}(x)<\epsilon_{j}, j=1, \cdots, N
$$

Se $\max \left\{p_{1}(x), \cdots, p_{N}(x)\right\}>0$, tomando $t=\frac{1}{2} \min \left\{\epsilon_{1}, \cdots, \epsilon_{N}\right\} / \max \left\{p_{1}(x), \cdots, p_{N}(x)\right\}$ temos que $t p_{j}(x)<\epsilon_{j}$ para $j=1, \cdots, N$, então de $(A .3)$ segue-se que $(A .2)$ é valido com $C=2 / \min \left\{\epsilon_{1}, \cdots, \epsilon_{N}\right\}$.

Se $p_{j}(x)=0$, para todo $j=1, \cdots, N$ então $t p_{j}(x)<\epsilon_{j}, \forall t>0$. Logo, $q(x)=0$ e $(A .2)$ é valido trivialmente, com o mesmo $C$.

Reciprocamente, é obvio que $q$ é continua em 0 quando (A.2) é verdadeiro. Por outro lado, desde que $q$ é uma seminorma temos que

$$
|q(x)-q(y)| \leq q(x-y) \quad x, y \in X
$$

Assim a seminorma que é continua em 0 é continua em $X$.

Como um corolário imediato desta proposição temos

Corolário A.2: A topologia de $X$ também é gerado pela família de todas as seminormas continuas.

Proposição A.3: Suponha que a familia $\mathcal{P}$ de seminormas que geram a topologia de $X$ tem a seguente propriedade. Se $p_{1}, p_{2} \in \mathcal{P}$, então existe $p \in \mathcal{P}$ tal que $p_{1}(x) \leq p(x)$ e $p_{2}(x) \leq p(x), \forall x \in X$. Então uma forma linear $\varphi$ em $X$ é continua se, e somente se, existe $p \in \mathcal{P}$ e uma constante $C>0$ tal que

$$
|\langle\varphi, x\rangle| \leq C p(x), x \in X
$$

Demonstração. Como $\varphi$ é contínua, temos que aplicação $x \rightarrow|\langle\varphi, x\rangle|$ é uma semi-norma contínua. Logo, pela Proposição $A .1$ temos que existem seminormas $p_{1}, \cdots, p_{N} \in \mathcal{P}$ e $C>0$ tal que

$$
q(x) \leq C \max \left\{p_{1}(x), \cdots, p_{N}(x)\right\}, x \in X
$$


$\log$, pela propriedade que tem $\mathcal{P}$, existe $p \in \mathcal{P}$ tal que $p_{j}(x) \leq p(x)$, para $j=1, \cdots, N$ e $x \in X$. Por tanto segue-se $(A .4)$. O recíproco é imediato. 


\section{Referências Bibliográficas}

[1] Bott, R.; Tu, L.; Diferential Forms in Algebraic Topology. Graduate Texts in Mathematics, No. 82. Springer-Verlag Berlin Heidelberg, New York. (1991).

[2] Bowen, R.; Equilibrium states and the ergodic theory of Anosov diffeomorphisms. Lecture Notes in Math. 470. Springer-Verlag 1975.

[3] Bowen, R.; Periodic points and measures for Axiom A diffeomorphisms. Trans. Amer. Math. Soc., 154:377?397, 1971.

[4] Bowen, R.; Topological Entropy and Axiom A. Proc. of Symp. on Pure Math. XIV (1968), 23-41.

[5] Bowen, R.; Markov partitions for Axiom A diffeomorphisms, Amer. Journal of Math. 92 (1970), 907-918.

[6] Camacho, C.; Lins Neto, A.; Teoria geométrica das folheações. Projeto Euclides, IMPA. Rio de Janeiro, 1979.

[7] Candel, A. y Conlon, L. Foliations I. American Mathematical Society. EUA, 2000.

[8] DeRham, G.; Variétés différentiables. Formes, courantes, formes harmoniques. Herman. Paris, 1955.

[9] Garnett, L.; Foliations and mesuares harmonic along the leaves of a foliations and the ergodic theorem. Tesis, I.H.E.S. Bures-sûr-Yvette, 1980. Addison-Wesley Publishing Co., Reading, Mass.-London-Don Mills, Ont. (1969).

[10] Godbillon, C.; Feuilletages. Études géométriques. Birkhäuser verlag. Basel-BostonBerlin, 1991.

[11] Guillemin, V.; Pollack, A.; Diferential Topology. Prentice-Hall, 1974. 
[12] Katok, A.,Hasselblatt, B.; Intoduction to the modern theory of Dynamical Systems Cambridge Univ. Press, 1995.

[13] Lima, E.; Variedades Diferenciaveis, Monografias de Matemática \#15, IMPA, 1973.

[14] Mañe, R.; Ergodic theory and differentiable dynamics. Springer Verlag, 1987.

[15] Meyer, C.; Matriz analysis and applied linear algebra. Society for Industrial and Applied Mathematics, (SIAM), 2000.

[16] Oliveira, K.; Viana, M.; Fundamentos da Teoria Ergodica. (Preprint).

[17] Plante, J. F.; Foliations with mesuare preserving holonomy, Ann. of Math. 102, 327$361,1975$.

[18] Robinson, C.; Dynamical Systems. Stability, symbolic dynamics, and chaos, CRC Press, 1995.

[19] Ruelle, D.; Sullivan, D.; Currents, flows and diffeomorphisms, Topology 14, 319-324, 1975 .

[20] Schwartzmann, S. Asymptotic cycles. Ann. of Math. 66 (1957), 270-284.

[21] Shub, M.; Global Stability of Dynamical Systems, Springer-Verlag, 1987.

[22] Spivak, M.; A Comprehensive Introduction to Differential Geometry, Vol. 1, 3rd ed.,Publish or Perish, Houston, 2005.

[23] Sullivan, D.; Cycles for the dynamical study of foliated manifolds and complex manifolds. Inv. Math., 36, 1976, pp. 225-255. 